\title{
Multi-omic profiling of primary mouse neutrophils predicts a pattern of sex- and age-related functional regulation
}

\author{
Ryan J. Lu',2, Shalina Taylor ${ }^{3}$, Kévin Contrepois ${ }^{4}$, Minhoo Kim ${ }^{1}$, Juan I. Bravo ${ }^{1,2}$, \\ Mathew Ellenberger ${ }^{1}{ }^{4}$, Nirmal K. Sampathkumar ${ }^{1,8}$ and Bérénice A. Benayoun $\oplus^{1,5,6,7 凶}$
}

\begin{abstract}
Neutrophils are the most abundant human white blood cell and constitute a first line of defense in the innate immune response. Neutrophils are short-lived cells and thus the impact of organismal aging on neutrophil biology, especially as a function of biological sex, remains poorly understood. Here, we describe a multi-omic resource of mouse primary bone-marrow neutrophils from young and old female and male mice, at the transcriptomic, metabolomic and lipidomic levels. We identify widespread regulation of neutrophil 'omics' landscapes with organismal aging and biological sex. In addition, we leverage our resource to predict functional differences, including changes in neutrophil responses to activation signals. This dataset represents a large multi-omics resource for neutrophils across sex and ages and identifies neutrophil characteristics that could be targeted to improve immune responses as a function of sex and/or age.
\end{abstract}

$\mathrm{N}$ eutrophils are the most abundant cells in human blood, representing $50-70 \%$ of leukocytes ${ }^{1}$. These cells are continually produced in the bone marrow and released into circulation $^{2,3}$. Neutrophils are short-lived, with estimated cellular lifespans of $\sim 10-18 \mathrm{~h}$ once released in the bloodstream ${ }^{4,5}$, although they can survive longer ${ }^{4,6}$. Throughout their cellular lifespan, neutrophils undergo 'neutrophil aging, a process distinct from organismal aging $^{3,7}$. Neutrophils perform many key functions, including production of antimicrobial granules and of 'neutrophil extracellular traps' (NETs) ${ }^{3,8}$. Although neutrophils are essential against infections as the 'first line of defense', their aberrant activation can also aggravate inflammatory disease ${ }^{2,3}$. Indeed, emerging evidence suggests that neutrophils play important roles in chronic inflammation?

Organismal aging in mammals is characterized by systematic immune dysfunction and chronic inflammation, a phenomenon dubbed 'inflamm-aging ${ }^{10}$, which may be partially mediated by dysfunction of innate immune cells ${ }^{11}$. Indeed, emerging evidence suggests that neutrophils from aged organisms are dysfunctional ${ }^{12-16}$. Observed age-related dysfunctions in neutrophils include decreased NETosis in tumor necrosis factor (TNF)- $\alpha$-primed conditions ${ }^{13,14}$, reduced chemotaxis to sites of inflammation ${ }^{15}$ and secretion of intracellular granule proteases ${ }^{15,17}$. Although gene expression changes throughout the lifespan have been reported across many cell types ${ }^{18,19}$, how organismal aging (rather than 'daily' cell aging) affects neutrophil landscapes remains largely unknown.

Females and males present with many biological differences, which may underlie lifelong health disparities between sexes ${ }^{20}$ and could result from differential 'omic' regulation ${ }^{21,22}$. Although transcriptional differences between young female and male murine neutrophils have been profiled by ImmGen ${ }^{22}$, how these differences interplay with organismal aging and whether they are accompanied by other phenotypical differences remains largely unknown. Accumulated studies suggest that aspects of neutrophil biology are sex dimorphic, such as inflammatory mediator production ${ }^{9}$ or functional modulation by testosterone ${ }^{23}$. However, the pathways underlying sex dimorphism in neutrophils, as well as the extent of sex dimorphism, are still unclear.

To gain insights into how neutrophils are regulated as a function of age and sex, we generated a multi-omic resource covering transcriptome, metabolome and lipidome profiling of primary bone-marrow mouse neutrophils. We identified widespread agerelated and sex-dimorphic 'omic' regulation, including transcriptional regulation of chromatin-related pathways. Using the assay for transposase-accessible chromatin using sequencing (ATACseq), we showed that remodeling of chromatin-related pathways was associated with overall differences in the chromatin architecture of neutrophils from young versus old and female versus male mice. Consistently, we observed age- and sex-linked differences in NETosis inducibility. Machine learning showed that specific factors could predict age-related and sex-dimorphic gene regulation in neutrophils. Finally, we leveraged our resource and predicted sex differences in serum levels of neutrophil elastase in control and sepsis-like conditions.

\section{Results}

Multi-omics of bone-marrow neutrophils with age and sex. To understand how neutrophils are regulated as a function of age and sex, we obtained primary bone-marrow neutrophils from young (4-5-month-old) and old (20-21-month-old) C57BL/6Nia female and male mice (Fig. 1a). Primary neutrophils were isolated from

'Leonard Davis School of Gerontology, University of Southern California, Los Angeles, CA, USA. ${ }^{2}$ Graduate Program in the Biology of Aging, University of Southern California, Los Angeles, CA, USA. 'Departments of Pediatrics and of Medicine, Stanford University School of Medicine, Stanford, CA, USA. ${ }^{4}$ Department of Genetics, Stanford University, Stanford, CA, USA. ${ }^{5}$ USC Norris Comprehensive Cancer Center, Epigenetics and Gene Regulation, Los Angeles, CA, USA. ${ }^{6}$ Molecular and Computational Biology Department, USC Dornsife College of Letters, Arts and Sciences, Los Angeles, CA, USA. ${ }^{7}$ USC Stem Cell Initiative, Los Angeles, CA, USA. ${ }^{8}$ Present address: UK-Dementia Research Institute, Institute of Psychiatry, Psychology and Neuroscience, Basic and Clinical Neuroscience Institute, Maurice Wohl Clinical Neuroscience Institute, King's College London, London, UK. 凶e-mail: berenice.benayoun@usc.edu 
a
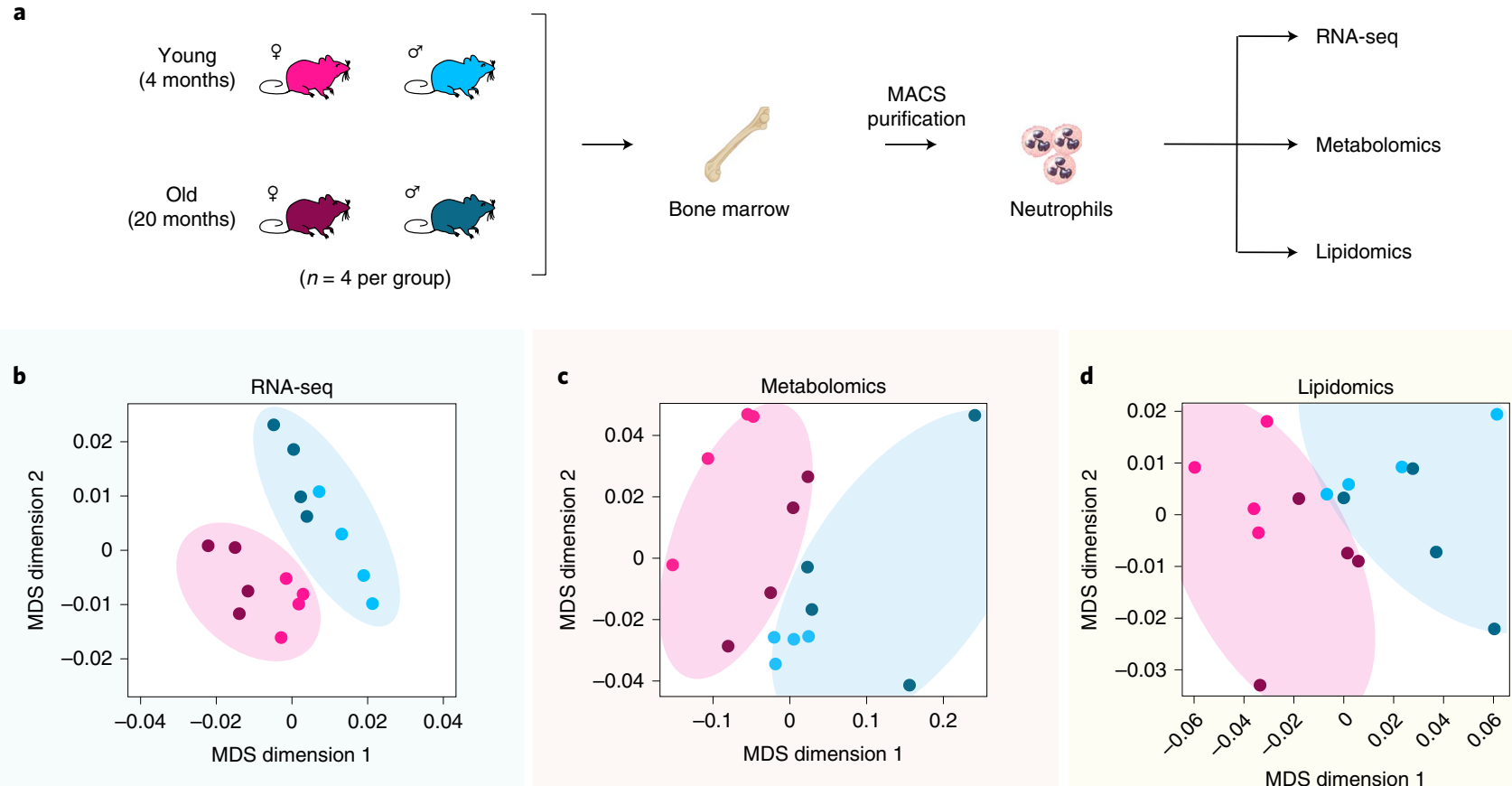

e Heat map of sex-dimorphic gene expression

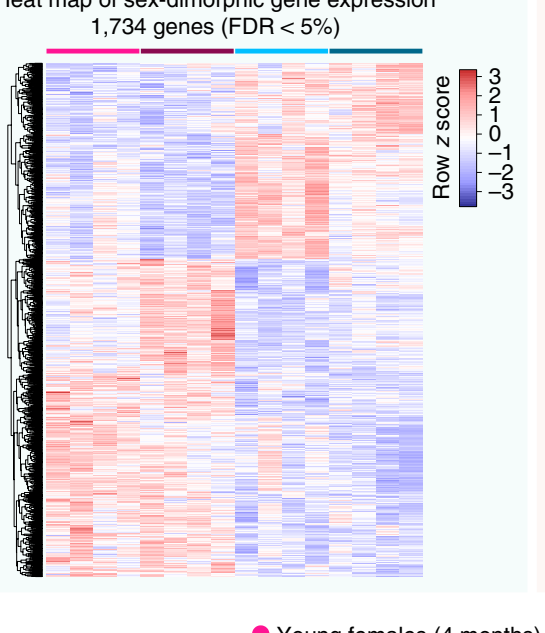

f

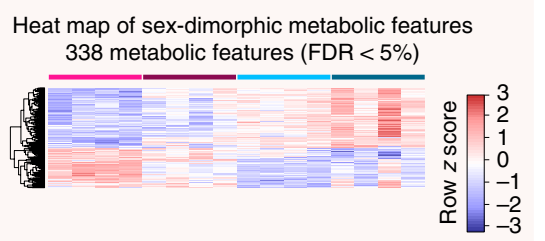

g

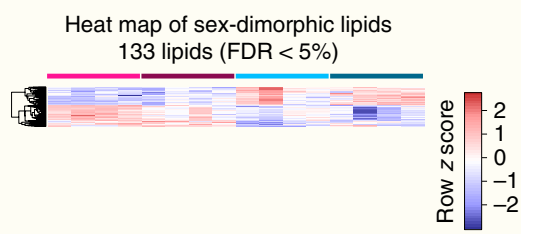

- Young females (4 months)

Old females (20 months)

- Young males (4 months)

Old males (20 months)

Fig. 1| A multi-omic analysis of primary mouse bone marrow neutrophils during aging and with respect to sex. a, Experimental setup scheme. b-d, MDS analysis results of RNA expression by RNA-seq (b), untargeted metabolomics (c) or targeted lipidomics (d). e-g, Heat map of significant (FDR <5\%) sexdimorphic genes (e), metabolic features (f) or lipid species (g). Significance of gene regulation by RNA-seq was calculated by DESeq2 and significance of metabolic features or lipid species regulation were calculated by limma.

bone marrow using magnetic-activated cell sorting (MACS) and profiling was then performed on purified neutrophils: (1) transcriptome profiling by RNA-sequencing (RNA-seq), (2) metabolomic profiling by untargeted liquid chromatography coupled with mass spectrometry (LC-MS) and (3) lipidomic profiling by targeted MS (Fig. 1a).

As a first-level analysis to evaluate the similarity of our datasets, we utilized multidimensional scaling (MDS). MDS analysis for RNA-seq, metabolomics and lipidomics datasets showed clear separation of samples by animal sex, regardless of age (Fig. 1b-d). In contrast, although young and old samples separated within each sex, global separation by age regardless of sex was not clearly observed for each omics (Fig. $1 \mathrm{~b}-\mathrm{d}$ ). To better understand the nature of differences between neutrophils from young versus old (with sex as a covariate) and female versus male animals (with age as a covariate), we identified transcriptional, metabolic and lipidomic features with significant age- or sex-related regulation at a false discovery rate (FDR) $<5 \%$ using multivariate linear modeling (Fig. 1e-g, Extended Data Fig. 1a-f and Supplementary Table 1a-f). We quality-checked our dataset for appropriate expression of sex-specific genes (Extended Data Fig. 1b,c). Finally, we confirmed differential gene expression trends between groups using a small replicate RNA-seq cohort (Extended Data Fig. 2a,b) and comparing our data with published datasets from female versus male mouse spleen neutrophils ${ }^{22}$ and human blood neutrophils $^{24}$ (Extended Data Fig. 2a). Thus, our analyses suggest that observed transcriptional differences with respect to organismal age and sex in neutrophils are robust. 
Consistent with MDS, we identified genes, metabolic features and lipids with significant differential regulation with respect to sex (FDR < 5\%; Fig. 1e-g, Extended Data Fig. 1a-d and Supplementary Table 1b,d,e). Differentially expressed genes with respect to sex were located throughout the genome, suggesting that sex-dimorphic gene expression was not just a byproduct of genomic location (Extended Data Fig. 1a,e). Notably, we observed no biases in the purity of MACS-purified neutrophils across groups (Extended Data Fig. 3a,b) and no difference in neutrophil abundance in bone marrow (Extended Data Fig. 3c) across groups. Thus, sex-dimorphic and age-related 'omic' phenotypes are unlikely to result from a systematic purification bias between groups.

Although aging led to clear changes in neutrophil gene expression profiles (Extended Data Fig. 1a,f and Supplementary Table 1), few to no metabolomic and lipidomic changes were observed (Extended Data Fig. 1a and Supplementary Table 1c,f). This is consistent with overall poorer age-related separation observed for metabolomics and lipidomics (Fig. 1c,d). Higher biological variability in metabolites and lipids might explain the lack of changes with age. Alternatively, it is possible that the paucity of age-related metabolic and lipidomic differences may stem from biological specificities of neutrophils (such as short cellular lifespan). When female and male neutrophils were analyzed separately, we found that the transcriptional impact of aging on neutrophils correlated strongly between sexes (Spearman Rho $=0.593$ and $P \sim 0$ in significance of correlation test; Extended Data Fig. 1g). Thus, although neutrophils show clear sex differences throughout life, the trajectories of neutrophils with aging are highly similar regardless of sex. To note, we observed a greater number of transcriptional changes with organismal age in male versus female neutrophils (Extended Data Fig. 1h), suggesting that male neutrophils are more susceptible to aging. Together, our results indicate that neutrophils are highly sex dimorphic at the molecular level and that these sex differences persist (or may be amplified) with organismal aging.

Finally, as bone-marrow neutrophils can be heterogenous, we evaluated the composition of MACS-purified neutrophils across groups (Extended Data Fig. 4a-d and Supplementary Table 2a,b). We leveraged flow cytometry markers to estimate the proportions of neutrophil progenitors (pre- and pro-neutrophils), immature versus mature neutrophils and fresh versus 'aged' neutrophils in our population. Neutrophil progenitors were extremely rare among MACSpurified cells (pre- and pro-neutrophils represent $<0.5 \%$ of cells; Extended Data Fig. 4a and Supplementary Table 2a) and are unlikely to drive large 'omic' differences. Notably, we did not observe significant differences in the presence of mature versus immature neutrophils across groups (Extended Data Fig. 4a,c and Supplementary Table 2). Finally, we observed a significant trend for the increased presence of senescent/aged neutrophils $\left(\mathrm{CD} 62 \mathrm{~L}^{-}\right)$among mature neutrophils (Extended Data Fig. 4a,d and Supplementary Table 2a). Our observation is consistent with a previous report of accumulation of senescent/aged neutrophils in old mice due to decreased clearance by efferocytosis ${ }^{25}$. Based on previous studies of senescent/ aged neutrophils, changes in the relative proportion of 'fresh' versus 'aged' neutrophils among bone-marrow neutrophils may have functional impacts on inflammation, chemotaxis and NETosis ${ }^{26,27}$.

Age-associated changes in chromatin pathways in neutrophils. We first focused on the impact of organismal aging on neutrophils using pathway enrichment analysis (Fig. 2a-c, Extended Data Fig. 5a,b and Supplementary Table 3). To note, we only analyzed age-related functional remodeling at the transcriptomic level, as metabolomics and lipidomics showed few changes with aging (Extended Data Fig. 1a).

We assessed the coordination of age-related changes byperforming a network analysis (Fig. 2a). The network was constructed using ageregulated genes and protein-protein interaction (PPI) information from IMEx/InnateDB. Our analysis revealed that significantly ageregulated genes form a clearly interconnected network (Fig. 2a). The strong interconnection of age-related genes is consistent with the notion that coordinated shifts occur in neutrophils with organismal aging, rather than stochastic changes and suggests that these genes are co-regulated or are involved in common processes.

We then asked which pathways were regulated with aging (Fig. 2b, Extended Data Fig. 5a,b and Supplementary Table 3). Immune-related pathways were significantly upregulated according to gene set enrichment analysis (GSEA) (Fig. 2b, Extended Data Fig. 5a,b and Supplementary Table $3 a-c)$ and ingenuity pathway analysis (IPA) (Supplementary Table 3e). This suggests that neutrophil-associated immunity in older animals could have different characteristics, consistent with age-related immune dysfunction ${ }^{10}$.

Notably, the top downregulated pathways with organismal aging were overwhelmingly related to chromatin and cell cycle (Fig. 2b, Extended Data Fig. 5a,b and Supplementary Table 3a-c,e). Specifically, 18 histone-encoding genes were significantly downregulated with aging (Supplementary Table 1a). We did not observe significant differences in the cell-cycle phase distribution of MACSisolated neutrophils across groups (Extended Data Fig. 6a,b), suggesting that differential expression of cell-cycle pathways is not a byproduct of differential proliferation.

Changes in regulation of chromatin components are especially relevant to neutrophil biology. Indeed, neutrophils have a unique chromatin organization ${ }^{28}$, with increased chromatin compaction or 'supercontraction' ${ }^{29}$. In addition, neutrophils can extrude their chromatin to produce NETs in response to pathogens ${ }^{30}$. The extrusion of chromatin directly participates in pathogen killing, notably thanks to antimicrobial properties of histones ${ }^{31}$. Chromatin relaxation can also be potentiated by nuclear-translocated granule-derived proteases (such as Elane), ultimately helping bacterial killing ${ }^{32}$. Finally, although neutrophils are post-mitotic, cell-cycle genes can control NET production $^{33}$. Thus, our analysis suggests that neutrophils may experience changes in chromatin organization with organismal aging, a feature that may impact their ability to respond to infection.

We also observed that several autophagy-related pathways were upregulated in old neutrophils (Fig. $2 \mathrm{~b}$ and Supplementary Table $3 \mathrm{~b}, \mathrm{c}, \mathrm{e})$. This is consistent with loss of proteostasis being a 'hallmark of aging ${ }^{34}$. Control of autophagy is critical for neutrophil differentiation $^{35}$, regulation of NET formation ${ }^{36}$ and of degranulation ${ }^{37}$.

To identify candidate upstream regulators, we investigated whether target genes of specific transcription factors (TFs) were differentially regulated with aging (Fig. $2 \mathrm{c}$ and Supplementary Table 3d). We obtained lists of TF target genes derived from ChEA, JASPAR or Gene Expression Omnibus (GEO) through the Harmonizome (Methods). Notably, we restricted our testing to TFs with detectable expression in neutrophils according to RNA-seq. To note, the GEO 'TF' set from Harmonizome includes several nonTF regulators (such as chromatin-remodeling enzymes and signaling receptors), which are referred to hereafter as TFs for simplicity. Consistent with functional pathway enrichments, we observed significant age-related downregulation of E2f7 targets, which are known to regulate cycle-related expression ${ }^{38,39}$ (Fig. 2c). Although E2f7 has not been studied in neutrophils, it is highly expressed in committed progenitors ${ }^{40}$. Targets of Foxol were also significantly downregulated with aging in neutrophils (Fig. 2c). Foxo TFs are the primary targets of aging-regulating insulin/insulin-like growth factor 1 signaling ${ }^{41}$ and Foxol regulates neutrophil-mediated bacterial immunity ${ }^{42}$. Finally, known targets of Padi4 were significantly downregulated with aging (Fig. 2c). Padi4 is a peptidylarginine deaminase, catalyzing histone citrullination, which plays a key role in NETosis in vivo ${ }^{43,44}$. Transcript levels of Padi4 were significantly upregulated with aging $\left(\mathrm{FDR}=6.9 \times 10^{-3}\right.$; Extended Data Fig. $5 \mathrm{c}$ and Supplementary Table 1b). Protein levels of Padi4 were also significantly upregulated with aging regardless of $\operatorname{sex}\left(P=8.5 \times 10^{-3}\right.$; 
a

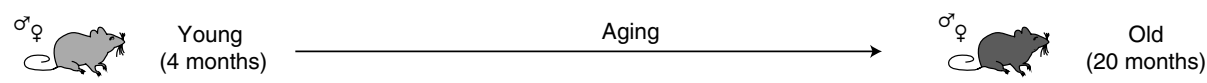

Putative PPI network affected by aging in neutrophils (IMEx)

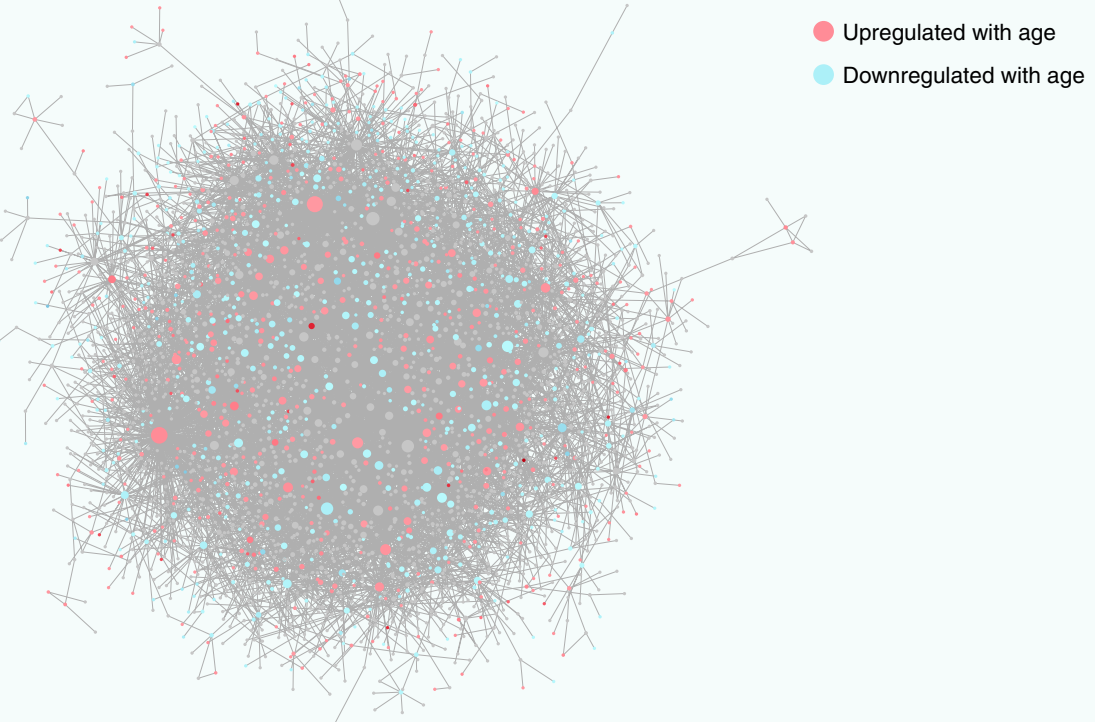

A tetrasaccharide-linker sequence is required for GAG synthesis Foxo-mediated transcription of cell cycle genes Autophagy Signaling by Nodal Erythropoietin activates Stat5 Chondroitin sulfate dermatan sulfate metabolism Rho GTPase cycle Effects of PiP2 hydrolysis Microautophagy IRE1- $\alpha$ activates chaperones Resolution of sister chromatid cohesion Cell cycle checkpoint

Sumoylation of DNA-replication proteins Interactions of REV with host cellular proteins Metabolism of noncoding RNA Cell cycle (mitotic) Transport of the SLBP-dependent mature mRNA Mitotic pro-metaphase Mitotic spindle checkpoint

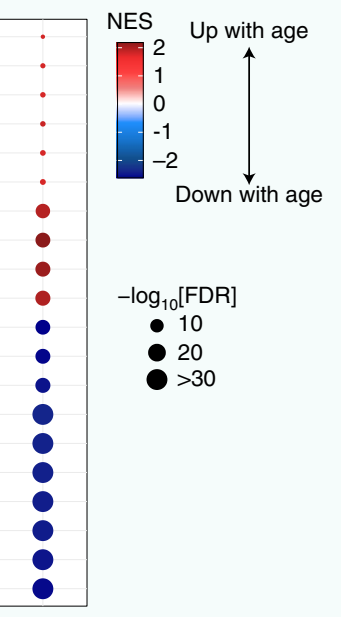

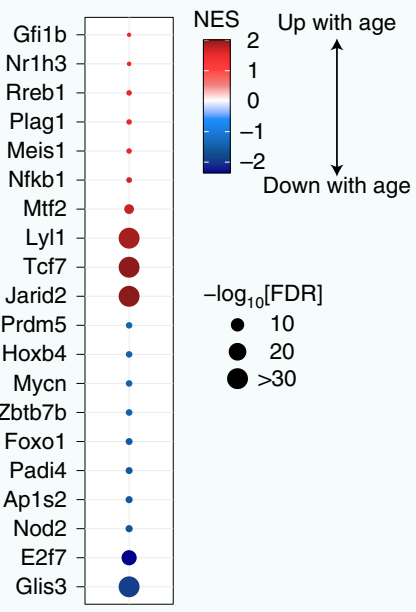

Fig. 2 | Regulated pathways in bone-marrow neutrophils during aging reveals downregulation of chromatin-related pathways. a, NetworkAnalyst predicted PPI network for significant age-regulated genes in neutrophils. Network edges are based on IMEx/InnateDB data, a knowledgebase specifically geared for analyses of innate immune networks ${ }^{119}$. Blue (red) shades are associated with decreased (increased) gene expression during aging. b,c, Top enriched gene sets from Reactome (b) and transcription factor targets (c) using GSEA for differential RNA expression. Only the top ten most-upregulated and top ten most-downregulated gene sets are plotted for readability. Full lists and statistics available in Supplementary Table 3. Also see Extended Data Fig. 5. Shown are pathways with FDR $<5 \%$. NES, normalized enrichment score.

Extended Data Fig. 5d,e). This is consistent with reports of histone citrullination associating to transcriptional repression ${ }^{45-47}$, although the directionality of regulation may depend on cofactors and cell types $^{48}$. Together, our functional pathway analysis suggests that major aspects of chromatin metabolism are shifting in aging bonemarrow neutrophils.

Sex dimorphism in chromatin pathways in neutrophils. We next analyzed the functional impact of neutrophil sex dimorphism at the transcriptomic, metabolomic and lipidomic levels (Fig. 3a-f, Extended Data Fig. 7a-c and Supplementary Table 4). We first assessed the interconnection of sex-dimorphic genes by constructing putative PPI networks for female versus male-biased gene expression (Fig. 3a,b). Sex-biased gene programs showed clear interconnection, suggesting coherent differences with likely functional impact (Fig. 3a,b). The top-connected node in the femalebiased gene network, Iqcb1, encodes a primary cilia component and has been linked to severe kidney disease ${ }^{49}$ (Fig. 3a). Notably, Irf8 was the top connected node in the male-biased gene network (Fig. 3b). Although not specifically studied in neutrophils, Irf8 is an interferon signaling-related TF that regulates myeloid fate determination, usually suppressing neutrophil differentiation ${ }^{50,51}$. In this context, Irf8 is likely to accomplish functions unrelated to myeloid differentiation (such as cytokine production) $^{52}$, as no significant changes in relative bone-marrow neutrophil abundances were observed (Extended Data Fig. 3c). 


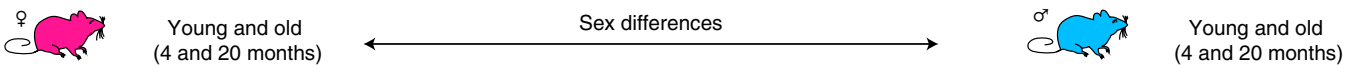

a Putative PPI network of female-biased expression in neutrophils (IMEx)

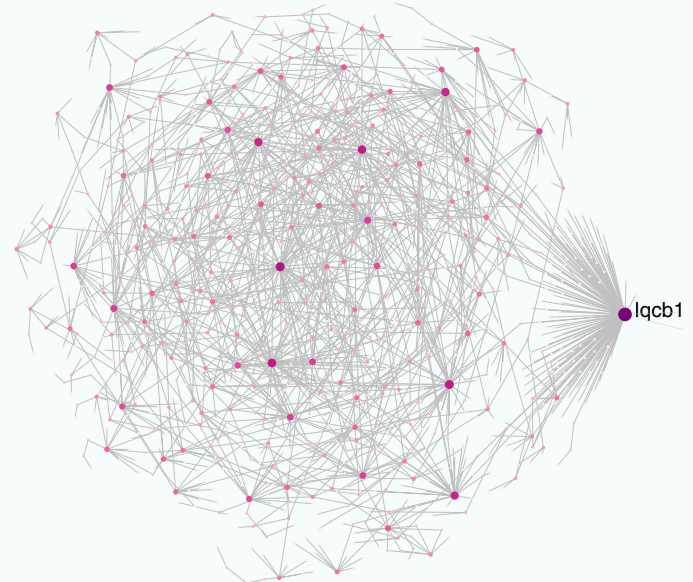

C Top GSEA enrichments for sex-regulation from RNA-seq (reactome)

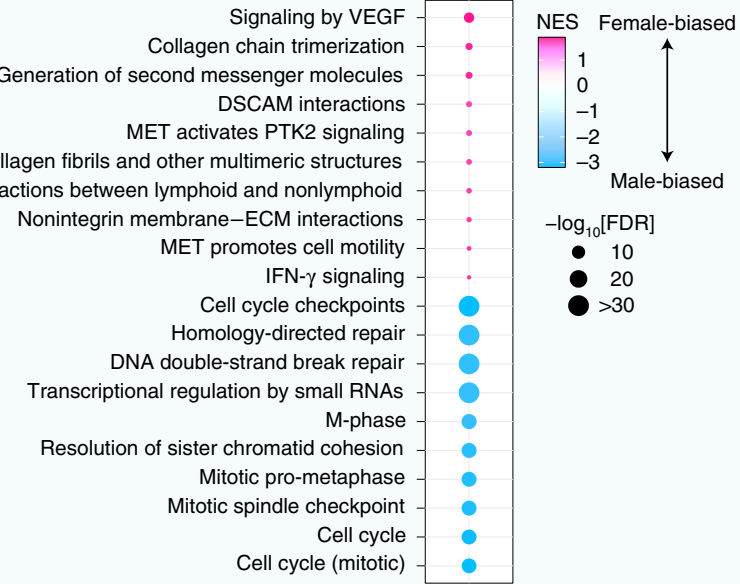

PSEA enrichments for sex regulation from metabolomics (KEGG)

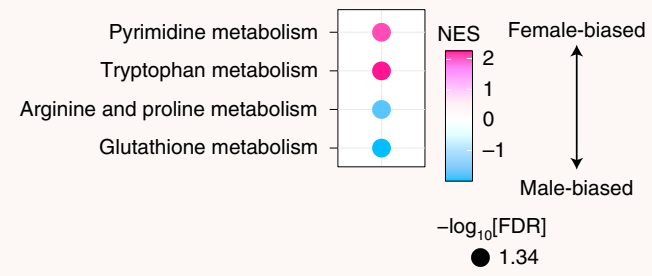

b Putative PPI network of male-biased expression in neutrophils (IMEx)

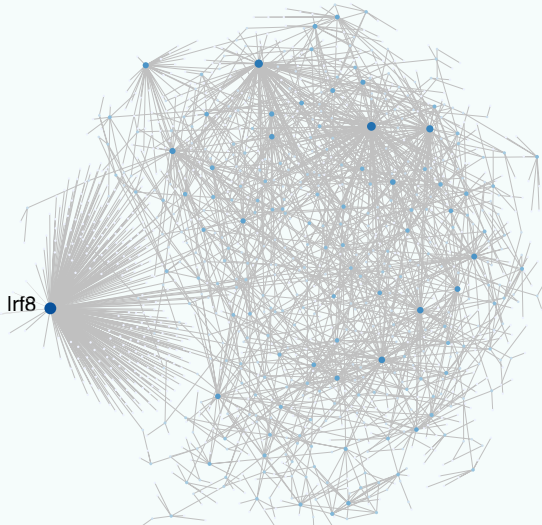

d Top GSEA enrichments for sex regulation from RNA-seq (TF targets)

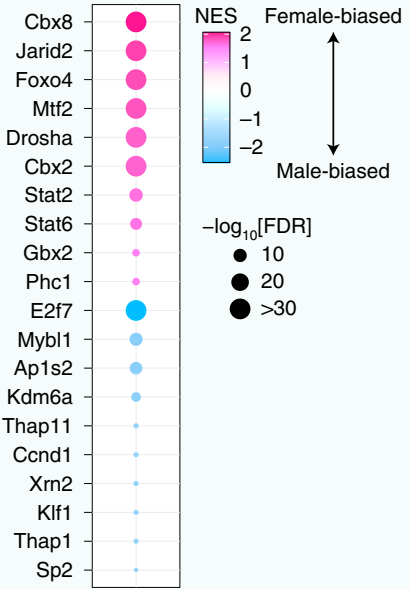

f Top LION enrichments for sex regulation from lipidomics

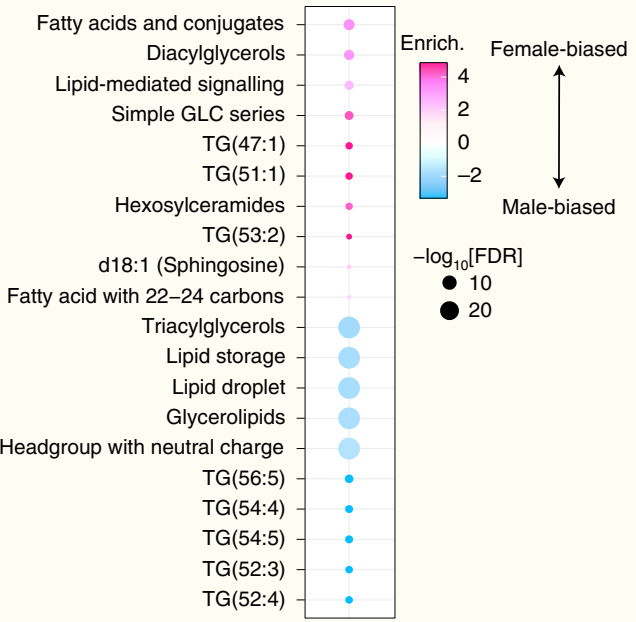

Fig. 3 | Sex-dimorphic pathways in bone-marrow neutrophils reveal differential regulation of chromatin-related pathways. a,b, NetworkAnalyst predicted PPI networks for genes displaying significant bias in gene expression by RNA-seq toward female (a) or male (b) neutrophils. Network edges are based on IMEx/InnateDB data, a knowledgebase specifically geared for analyses of innate immune networks ${ }^{119}$. $\mathbf{c}, \mathbf{d}$, Top enriched gene sets from Reactome (c) and transcription factor (TF) targets (d) using GSEA for differential RNA expression. DSCAM, down syndrome cell adhesion molecule; MET, MET proto-oncogene, receptor tyrosine kinase; VEGF, vascular endothelial growth factor. e, Phenotype set enrichment analysis (PSEA) for differential metabolite regulation. $\mathbf{f}$, Top LION functional enrichment analysis for differential lipid regulation. For $\mathbf{c}-\mathbf{f}$, the top ten most-upregulated and top ten mostdownregulated gene sets (if that many) are plotted for readability. Full lists and statistics available in Supplementary Table 4. Also see Extended Data Fig. 7. Shown are pathways with $\mathrm{FDR}<5 \%$. 
Next, we asked which pathways were regulated in a sex-dimorphic fashion in neutrophils (Fig. 3c, Extended Data Fig. 7a,b and Supplementary Table 4). Functional enrichment analysis revealed that significant female-biased pathways encompassed extracellular matrix (ECM) and cell surface-related pathways (Fig. 3c, Extended Data Fig. 7a,b and Supplementary Table $4 \mathrm{a}-\mathrm{d}$ ). Indeed, collagenencoding genes Col1a1 and Colla2 were expressed at higher levels in female neutrophils (Supplementary Table 1b). Collagen production, usually from fibroblasts, is a key event in fibrosis ${ }^{53}$ and neutrophils play an important role in the development of fibrotic lesions ${ }^{54}$. Alternatively, differential expression of cell-surface-related genes may also impact the ability of neutrophils to migrate through endothelial barriers ${ }^{55}$. Notably, autophagy-related gene sets were femalebiased in our dataset (Supplementary Table 4a,c,e). As mentioned above, autophagy control is critical for neutrophil biology $y^{35,36}$, including neutrophil degranulation ${ }^{37}$.

Chromatin- and cell-cycle-related pathways were overwhelmingly biased for higher expression in male neutrophils (Fig. 3c, Extended Data Fig. 7a,b and Supplementary Table 4a-c). Consistently, 21 histone-encoding genes showed significant malebiased expression (Supplementary Table 1b). The sex-dimorphic regulation of chromatin-related pathways suggests that sex may lead to differences in neutrophil chromatin metabolism.

We next investigated whether sex-dimorphic expression was correlated with predicted activity of TFs in neutrophils (Fig. $3 \mathrm{~d}$ and Supplementary Table 4d). Consistent with pathway analysis, targets of cell cycle-related E2f7 and Mybl ${ }^{39,56}$ were more highly expressed in male neutrophils (Fig. 3d). Female neutrophils showed higher expression of Foxo4 targets (Fig. 3d). Notably, the Insulin/Igf1 signaling pathway, which regulates Foxo TF activity, has been linked to multiple sex-dimorphic phenotypes ${ }^{57,58}$.

We next examined sex-dimorphism based on metabolomics and identified a number of pathways with significant sex-bias (Fig. 3e, Extended Data Fig. 7c and Supplementary Table 4f). Notably, metabolic pathways related to nucleotide metabolism were differentially regulated between sexes (Fig. 3e, Extended Data Fig. 7c and Supplementary Table 4f). Indeed, a number of sex-dimorphic metabolic peaks were predicted to represent nucleotide species (such as AMP, ATP, GMP; Supplementary Table 1d). Sex differences in nucleotide pools are consistent with transcriptomic observations of sex-dimorphism in (1) pathways directly related to nucleotide metabolism, which could lead to direct changes in nucleotide pools and (2) pathways responsive to nucleotide levels, such as signaling by Rho-GTPases, which can regulate neutrophil recruitment ${ }^{59}$ (Supplementary Table $4 \mathrm{a}-\mathrm{c}, \mathrm{e}$ ). Among nucleotides, the impact of adenine derivatives in neutrophils has been studied most extensively. ATP/ADP levels are regulated in neutrophils as a function of activation and 'cellular age' ${ }^{\prime 60}$. Adenine nucleotides from neutrophils exert anti-inflammatory effects ${ }^{61}$, increase endothelial barrier function, attenuate neutrophil adhesion to endothelial cells and modulate transendothelial migration ${ }^{62}$. Functional analysis of metabolomic data also suggested significant sex differences in amino-acid metabolism, such as tryptophan and arginine/proline metabolism (Fig. 3e and Extended Data Fig. 7c). Of note, arginine and tryptophan metabolism play important immunoregulatory roles $^{63}$; however, the final effect of differential metabolite levels from these pathways between sexes will need further investigation.

We then used the Lipid Ontology (LION) framework to analyze sex dimorphism in lipidomic profiles (Fig. 3f, Extended Data Fig. 1d and Supplementary Table 4g). Notably, male neutrophils were strongly enriched for triacylglycerols (TAGs) stored in lipid droplets, whereas female neutrophils were enriched for diacylglycerols (DAGs) (Fig. 3f, Supplementary Table 4g and Extended Data Fig. 1d). Consistently, genes associated with the Gene Ontology (GO) term 'negative regulation of lipid storage' were expressed at significantly higher levels in female neutrophils $\left(\mathrm{FDR}=4.33 \times 10^{-3}\right.$;
Supplementary Table 4c). Lipid droplets are crucial during neutrophil differentiation and as a source for inflammatory mediators $^{35,64}$. Adipose triglyceride lipase (Atgl), encoded by Pnpla2, regulates neutral lipid storage into lipid droplets in neutrophils ${ }^{65}$. Of note, Pnpla2 is expressed at higher levels in female neutrophils $(F D R=0.01$; Extended Data Fig. 7d and Supplementary Table 1b). Indeed, Atgl catalyzes the conversion of TAGs to DAGs, consistent with higher levels of DAGs in female neutrophils and of TAGs in male neutrophils (Fig. 3f, Extended Data Fig. 1d and Extended Data Fig. 7e). Lower levels of Pnpla2 (mimicking a 'masculinized' state) can lead to abnormal neutral lipid accumulation in neutrophils, increased chemotactic ability and reduced release of proinflammatory lipids ${ }^{65}$. In addition, Atg7 is crucial for generation of free fatty acids (FFAs) from lipid droplets in neutrophils and Atg7-deficient neutrophils show increased lipid-droplet storage ${ }^{35}$. Consistently, Atg7 is expressed at higher levels in female neutrophils (FDR =0.03; Extended Data Fig. 7d and Supplementary Table 1b). Finally, Lpin1 is crucial for synthesis of DAG from phosphatidic acid and plays a role in the regulation of inflammation ${ }^{66}$. Consistent with increased DAG levels, Lpin1 is also expressed at higher levels in female neutrophils $(F D R=0.03$; Extended Data Fig. 7d and Supplementary Table 1b). Thus, both lipidomics and transcriptomics data suggest that male versus female neutrophils have increased neutral lipid storage (or decreased usage) (Extended Data Fig. 7e). Because of the functional importance of lipid droplets, these observations are likely to have deep functional consequences on neutrophil function.

Finally, we performed an integrated analysis using IMPaLA, combining information from transcriptomics, metabolomics and lipidomics (Supplementary Table 1h). We observed joint enrichment of male-biased molecules in pathways linked to cell cycle, DNA metabolism and chromatin-related pathways (Supplementary Table $4 \mathrm{~h}$ ), further supporting the notion that neutrophil chromatin architecture may be regulated in a sex-dimorphic manner. In contrast, female-biased pathways were enriched for lipid metabolism (Supplementary Table $4 \mathrm{~h}$ ). These joint observations provide an integrated confirmation of our observations for individual 'omic' layers. Together, our analyses suggest that major aspects of neutrophils are regulated in a sex-dimorphic fashion and may ultimately underlie sex differences in immune responses.

Neutrophil chromatin organization changes with sex and age. Our enrichment analyses revealed that chromatin-related pathways were significantly modulated by sex and organismal age in neutrophils, which is expected to result in profound changes in chromatin architecture. Neutrophils hold their chromatin in a compacted polylobular nuclear architecture, which earned them the name of 'polymorphonuclear' cells ${ }^{28,29}$. More than just a gene expression regulatory layer, neutrophil chromatin directly participates in antimicrobial responses through $\mathrm{NETs}^{30,31}$. Thus, chromatin-metabolism differences could lead to profound changes in neutrophil biology. To directly evaluate neutrophil chromatin, we utilized ATAC-seq ${ }^{67}$, which takes advantage of adaptor-loaded Tn5 particles inserting into accessible chromatin, yielding information about local chromatin accessibility ${ }^{67}$ and higher-order organization ${ }^{68}$.

To evaluate chromatin landscapes, we isolated neutrophils from an independent cohort of young (4-5-month-old) and old (20-21-month-old) C57BL/6Nia female and male mice and performed ATAC-seq (Fig. 4a and Extended Data Fig. 8a-h). In contrast with transcriptomic, metabolomic and lipidomic data, MDS analysis on ATAC-seq showed that aging led to better sample separation than sex when examining local differences in chromatin accessibility (Extended Data Fig. 8d). In addition, there were substantially more regions with age-related versus sex-dimorphic differential accessibility (Extended Data Fig. 8e-h), consistent with numbers of differentially expressed genes with respect to age and sex 
(Extended Data Fig. 1a). Notably, enriched GO terms associated with differentially accessible regions with respect to organismal age (FDR $<5 \%)$ were mostly associated with regions with increased accessibility and encompassed terms related to immune response (Supplementary Table 3f). Significantly enriched GO terms for differential regions with respect to sex $(\mathrm{FDR}<5 \%)$ were all femalebiased and featured terms related to MAPK signaling and chromatin (Supplementary Table 4i).

As enrichment analyses suggested that overall aspects of chromatin are differentially regulated with organismal age and sex, we leveraged NucleoATAC ${ }^{68}$ to analyze neutrophil chromatin architecture. Notably, nucleosome 'v-plots' generated revealed a region at $\sim 145-150$ bp that captured more reads in male versus female neutrophils, regardless of animal age, suggesting global differences in nucleosome packaging (Extended Data Fig. 8a). Next, we leveraged indicators linked to chromatin compaction and organization around nucleosomes: (1) nucleosome occupancy, which indicates how frequently a position is occupied by a nucleosome across cells, where higher occupancy is associated to tighter chromatin structure, (2) nucleosome fuzziness, which indicates how well positioned the nucleosome is, where fuzzier nucleosomes correspond to looser chromatin and (3) inter-dyad distance, which measure DNA length between neighboring nucleosomes, with increased distance associated to looser chromatin.

Occupancy metaplots around transcriptional start sites (TSSs) of expressed genes revealed that, regardless of age, male neutrophils showed higher median nucleosomal occupancy compared to female neutrophils (Fig. 4b). Similarly, regardless of sex, aging was associated to increased median nucleosomal occupancy (Fig. 4c). More generally, male neutrophils had a slight, but significant, increase in nucleosomal occupancy compared to female neutrophils and median nucleosomal occupancy also slightly increased with organismal age (Fig. 4d and Extended Data Fig. 8i). We observed decreased nucleosome fuzziness in male versus female neutrophils, as well as in old versus young neutrophils (Fig. 4e). Finally, we observed shorter inter-dyad distance in male versus female, as well as old versus young neutrophils (Fig. 4f). Together, small (but consistent) increases in occupancy, decreases in fuzziness and decreases in inter-dyad distance, are indicative of an overall more compacted chromatin architecture in male versus female and old versus young neutrophils. Finally, when analyzing ATAC-seq fragments at accessible regions (including subnucleosomal fragments), we observed lower median fragment length in male and old neutrophils (Extended Data Fig. 8j). While this observation may seem contradictory, overall shorter ATAC-seq fragments in the libraries may result from relatively 'more accessible' nucleosomefree regions in the context of overall tighter chromatin.

To independently evaluate chromatin compaction, we leveraged our RNA-seq to examine transcription of repeats (Extended Data Fig. 8k,l and Supplementary Table 1g,h). Eukaryotic genomes contain large proportions of repeats, including transposons, whose transcription is usually tightly repressed through compaction $^{69}$. Consistent with a more compact chromatin architecture, male neutrophils showed lower transcription of repeats versus females (350 female-biased elements versus 12 male-biased; Extended Data Fig. 81 and Supplementary Table 1h). Similarly, old neutrophils also showed lower transcription of repeats versus young neutrophils (115 elements downregulated versus 27 upregulated with age; Extended Data Fig. 8k and Supplementary Table $1 \mathrm{~g})$. Interestingly, chromatin decompaction is a limiting step of NETosis $^{70}$, suggesting that baseline differences in chromatin compaction in neutrophils of different ages and sex may impact the dynamics and timeline of NETosis. However, chromatin profiling alone cannot predict the direction in which NETosis could be affected.

Based on transcriptome and ATAC-seq results, we predicted that NETosis inducibility should be regulated as a function of sex and organismal age. We evaluated NETosis inducibility in neutrophils isolated from young versus old, female versus male mice (Fig. 4g-i). We used flow cytometry to quantify cells with extracellularized DNA (NETosed or NETosing cells), after a $2 \mathrm{~h}$ incubation in the presence of phorbol 12-myristate 13-acetate (PMA), a known activator of NETosis, compared to vehicle (Fig. 4g). We first evaluated NETosis induction on unstimulated neutrophils (Fig. 4h). NETosis inducibility was significantly increased with organismal age in female cells $\left(P=4.5 \times 10^{-5}\right)$, while higher animal-to-animal variability and no significant change were observed in male cells (Fig. 4h). Young male neutrophils showed higher NETosis capacity than young female neutrophils $(P=0.057)$, a trend reversed with organismal aging, with old female neutrophils showing highest NETosis inducibility ( $P=0.029$; Fig. 4 h).

Increased NETosis in neutrophils from older female mice may seem contradictory with previous reports of decreased NETosis with organismal aging ${ }^{13,14}$. To note, previous studies evaluated NETosis after in vitro TNF- $\alpha$ priming ${ }^{13}$ or in conditions known to mimic TNF- $\alpha$ priming ${ }^{14,71}$ and did not evaluate sex as a biological variable. TNF- $\alpha$ is a proinflammatory cytokine and a potent inducer of NETosis ${ }^{13}$. To evaluate how TNF- $\alpha$ priming may impact NETosis across groups, we also performed NETosis experiments on cells pretreated with $10 \mathrm{ng} \mathrm{ml}^{-1}$ TNF- $\alpha$ (Fig. 4i). Although TNF- $\alpha$ priming

Fig. 4 | ATAC-seq analysis reveals age- and sex-related differences in the chromatin architecture of bone-marrow neutrophils. a, Setup scheme for ATAC-seq experiment. b,c, Metaplot analysis of median nucleosome occupancy (as calculated by NucleoATAC) around the TSS of neutrophil-expressed genes, to analyze sex- (b) or age-related (c) differences in neutrophil nucleosome occupancy. Note that increased occupancy is observed in male compared to female neutrophils, as well as in old compared to young neutrophils. Significance of the difference between median occupancy profiles at TSSs assessed using a two-sided Kolmogorov-Smirnov goodness-of-fit test for b,c. d, Box plot of nucleosome occupancy as calculated by NucleoATAC for high-confidence nucleosomes across groups. Also see Extended Data Fig. 8i. e, Box plot of nucleosome fuzziness as calculated by NucleoATAC for highconfidence nucleosomes across experimental groups. f, Box plot of nucleosome inter-dyad distance as calculated by NucleoATAC across experimental groups. The horizontal red line in $\mathbf{d}-\mathbf{f}$ shows the median value in neutrophils from young females for ease of comparison. Significance in nonparametric two-sided Wilcoxon rank-sum tests is reported for $\mathbf{d}-\mathbf{f}$. Grey $P$ values represent differences between male versus female neutrophils in each age group: pink (blue) $P$ values represent age-related differences in female (male) neutrophils. Also see Extended Data Fig. 8i,j. g, Workflow for in vitro NETosis inducibility experiment. SYTOX Green was used to stain extracellular DNA, a proxy for NETosing cells. $\mathbf{h}$, Box plot of NETosis induction in naive neutrophils. Each point represents one animal, median of four technical replicates. Animals from four independent cohorts, $n=16-19$ per group (variation due to animal death prior to experiment and technical failures of the flow cytometer on some samples; $n=17$ for young males and females; $n=16$ for old females; $n=19$ for old males). $\mathbf{i}$, Box plot of NETosis induction in neutrophils, primed with $10 \mathrm{ng} \mathrm{ml}^{-1}$ mouse TNF- $\alpha$ for 10 min before NETosis induction. Each point represents one animal, median of four technical replicates. Animals from four independent cohorts, $n=17$ per group. Significance in nonparametric twosided Wilcoxon rank-sum tests are reported for $\mathbf{h}, \mathbf{i}$. Grey $P$ values represent differences between male versus female neutrophils in each age group; pink (blue) $P$ values represent age-related differences in female (male) neutrophils. For all box plots in $\mathbf{d}-\mathbf{f}, \mathbf{h}, \mathbf{i}$, the center line represents the sample median, the box limits consist of the 25 th and 75 th percentiles, the whiskers span $1.5 \times$ the interquartile range. For $\mathbf{d}-\mathbf{f}$, for readability and consistent with practices in genomics, outliers are not shown on the graph although they are always taken into account for the statistics. 
yielded noisier data, NETosis inducibility was blunted with organismal age in female cells stimulated with TNF- $\alpha$ (Fig. 4i). In contrast, TNF- $\alpha$-primed male neutrophils trended toward decreased inducibility of NETosis with organismal age ( $P=0.092$; Fig. 4i). In TNF- $\alpha$-primed conditions, young male versus female neutrophils no longer showed differences in NETosis inducibility, but old female neutrophils still showed the highest overall NETosis inducibility (Fig. 4i). Together, we find that NETosis is differentially modulated as a function of organismal age and sex, consistent with observed differences in chromatin architecture.

a

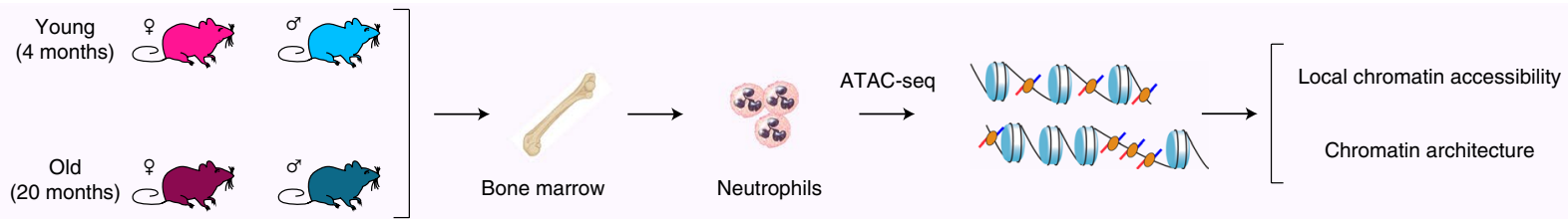

( $n=5$ per group)

b

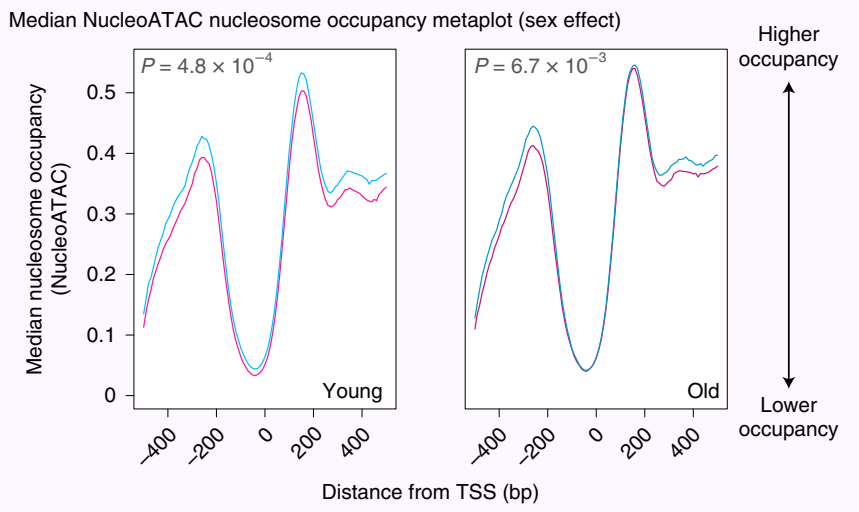

d

Nucleosome occupancy (high confidence)

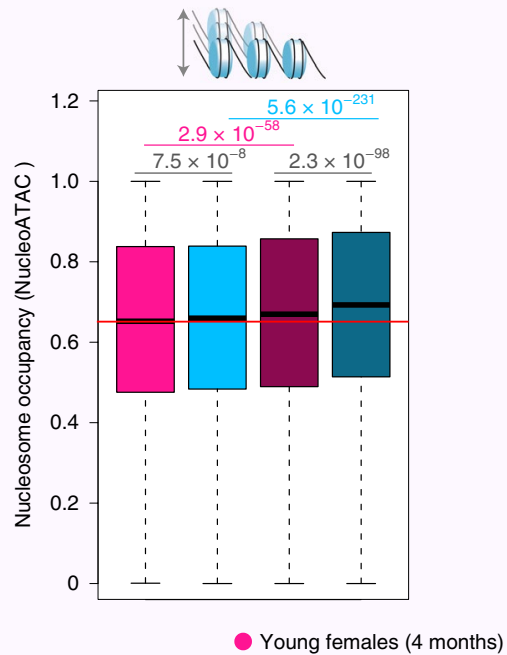

g

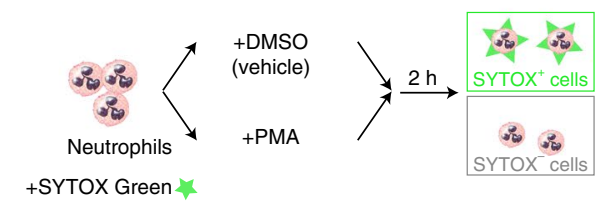

NETosis induction by PMA $=\frac{\text { PMA }\left(\% \text { SYTOX }{ }^{+} \text {cells }\right)}{\mathrm{DMSO}\left(\% \text { SYTOX }{ }^{+} \text {cells }\right)}$ e Nucleosome fuzziness

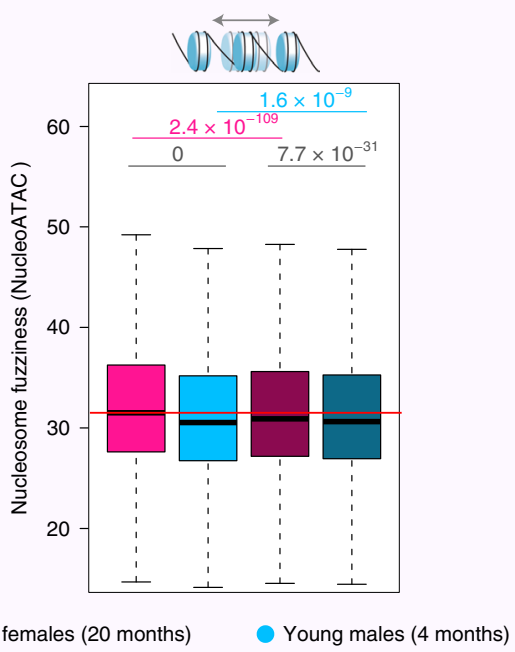

$\mathbf{h}_{\text {NETosis induction (naive neutrophils) }}$

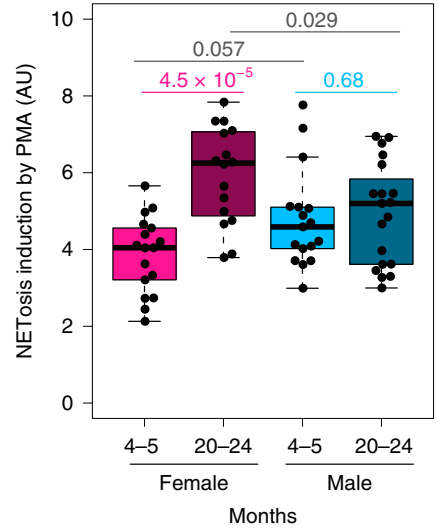

C Median NucleoATAC nucleosome occupancy metaplot (age effect)
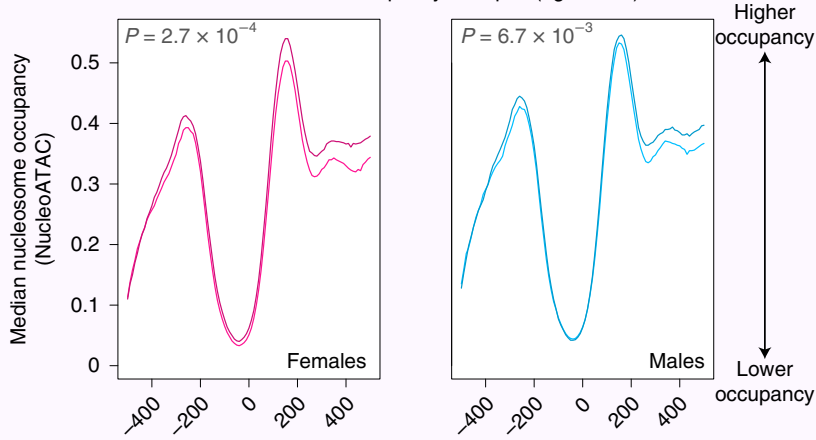

Distance from TSS (bp)

f Inter-dyad distance

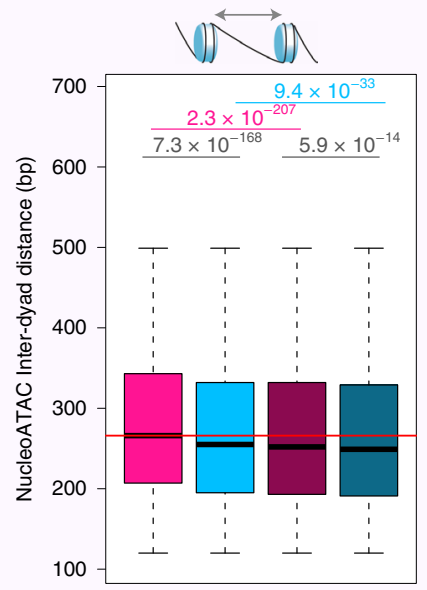

Old males (20 months)

i NETosis induction (TNF- $\alpha$ primed)

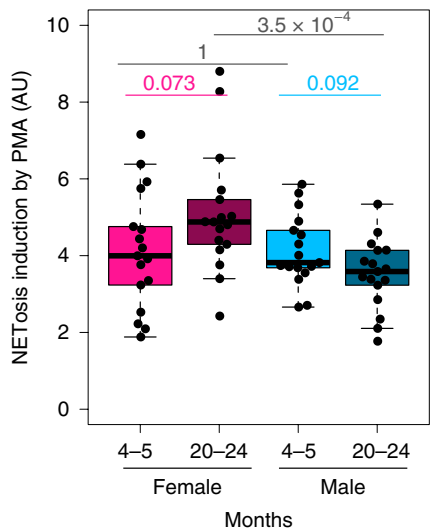




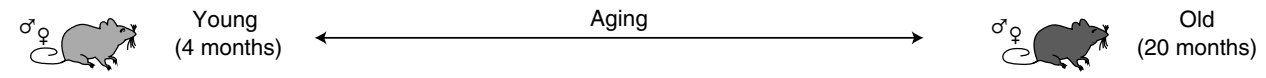

Scheme of classification pipeline (aging)

Genes

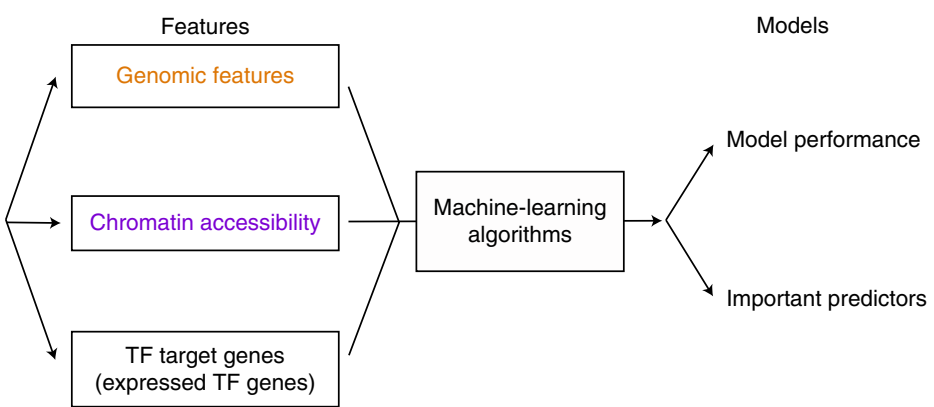

b Aging model performance (balanced accuracy)

Perfect
Random
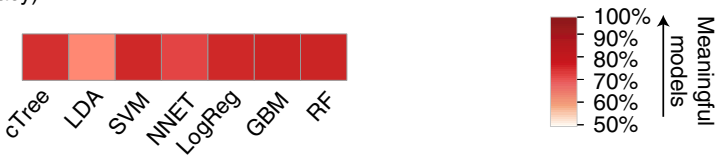

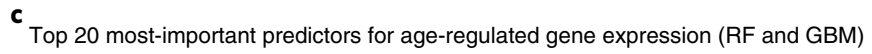

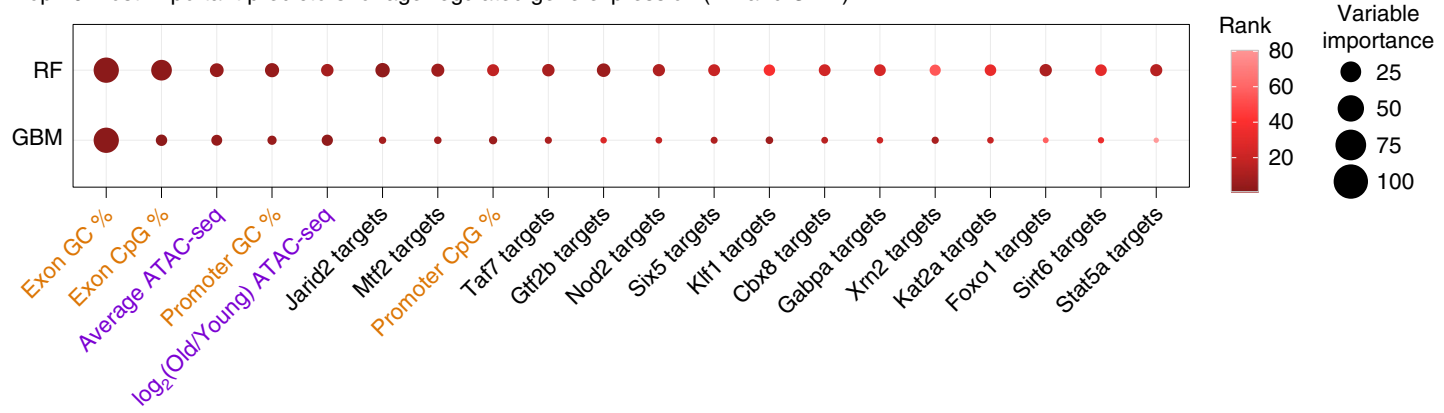

Fig. 5 | Machine-learning analysis reveals that age-regulated gene expression can be predicted by genomic and epigenomic features. a, Scheme of our machine-learning pipeline for upregulated versus downregulated genes in neutrophils with aging. $\mathbf{b}$, Balanced classification accuracy over different machine-learning model algorithms. The accuracy of the models is measured using held-out testing data. 'Random' accuracy illustrates the accuracy of a meaningless model ( $50 \%)$ and 'perfect' that of a model with no mistakes (100\%). All tests were more accurate than random (see other measures of model performance in Extended Data Fig. 9a-c and Supplementary Table 5a). CTree, conditional inference tree; LDA, linear discriminant analysis; SVM, support vector machine; NNET, neural network; LogReg, logistic regression. c, Top 20 most-important features from RF models and GBM models. A rank product approach was used to determine overall top predictive features from both models. High values for variable importance indicate most influential predictors.

Machine learning predicts features of neutrophils regulation. To understand whether age-related and sex-dimorphic gene expression can be predicted from genomic sequence, chromatin and/or regulation by TFs, we took advantage of machine learning (Figs. $5 \mathrm{a}-\mathrm{c}$ and $6 \mathrm{a}-\mathrm{c}$ and Extended Data Fig. 9a-f). Machine learning can help (1) to determine whether features contain information predictive of a state of interest (such as sex-dimorphic or age-regulated expression) and (2) to identify predictive features of such states. We used seven algorithms to learn models discriminating between (2) up- and downregulated expression with age and (3) male- or female-biased gene expression (conditional inference trees, linear discriminant analysis, neural networks, logistic regression, random forests (RFs), support vector machines and gradientboosting machines (GBMs)). The models were trained with features related to (1) genomic sequence (such as GC richness), (2) chromatin accessibility (such as changes in ATAC-seq promoter accessibility) and (3) TF target genes (the same as used with GSEA).

We first evaluated our ability to discriminate between genes upregulated versus downregulated during aging in neutrophils (Fig. 5a). Our models assigned genes to the correct transcriptional change with age $>64 \%$ of the time on testing data (Fig. $5 \mathrm{~b}$ and Supplementary Table 5a), significantly above random (50\%). High accuracy suggests that clear biological features differentiate between genes that tend to be upregulated versus downregulated with aging. To assess which features were most predictive of agerelated changes, we examined predictor contribution from RF and GBM models, which provide native evaluation of feature importance (Fig. $5 \mathrm{c}$ and Supplementary Table 5b). Consistent with the tight link between chromatin and gene expression ${ }^{72}$, the average promoter ATAC-seq signal (describing how 'open' the promoter is), as well as the fold difference in ATAC-seq signal between young and old neutrophils (age-related changes in openness) were among the top predictors for age-related changes (Fig. $5 \mathrm{c}$ and Supplementary Table $5 b)$. Key predictors also included CG/CpG content in the promoter and gene sequences (Fig. 5c), which is consistent with our previous work identifying promoter $\mathrm{CpG}$ content as a top predictor of age-related gene expression in mouse tissues ${ }^{18}$. Cytosines in $\mathrm{CpG}$ configuration are the primary targets of DNA methylation ${ }^{73}$. CpG DNA methylation (DNAme), catalyzed by DNA-methyltransferases (writers), can be removed by TET family proteins (erasers) and is interpreted through recognition by methyl-CpG binding proteins (readers $)^{73}$. Consistent with the notion that the predictive power 


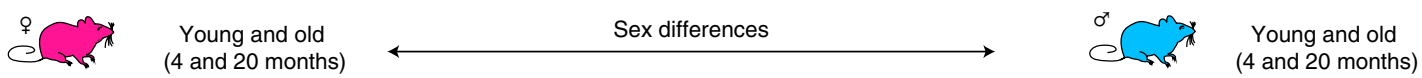

a Scheme of classification pipeline (sex dimorphism)

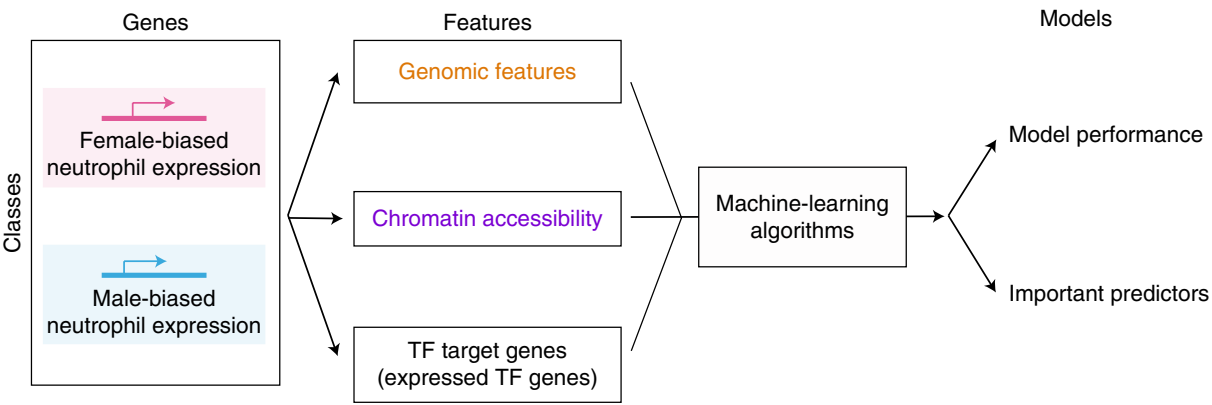

b Sex-dimorphism model performance (balanced accuracy)

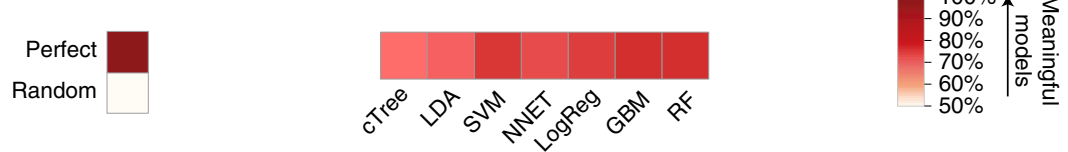

C Top 20 most-important predictors of sex-dimorphic gene expression (RF and GBM)

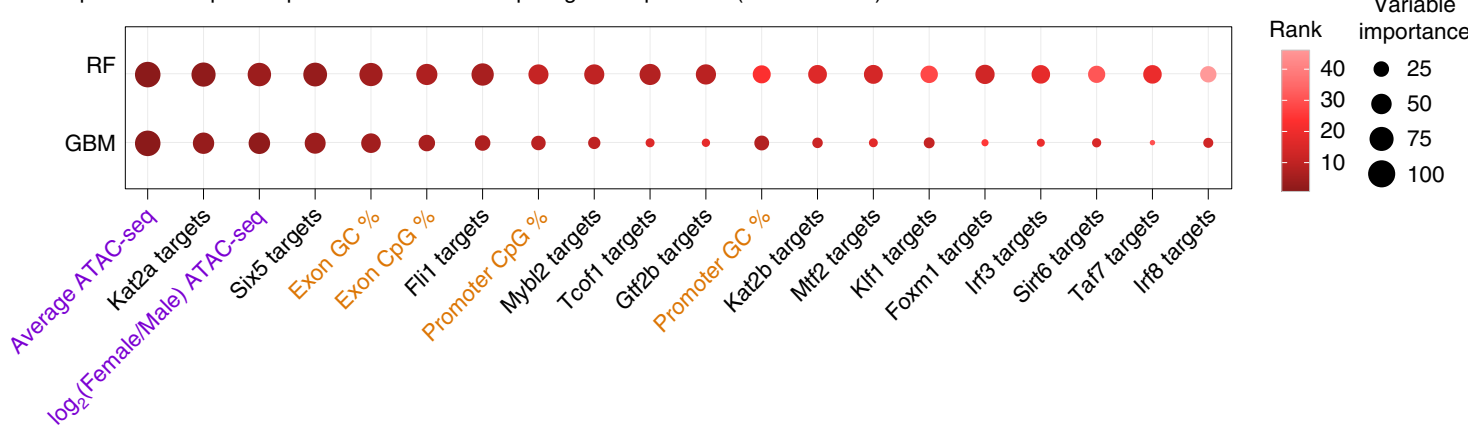

Fig. 6 | Machine-learning analysis reveals that sex-dimorphic gene expression can be predicted by genomic and epigenomic features. a, Scheme of our machine-learning pipeline for male versus female-biased gene expression in neutrophils. b. Balanced classification accuracy over different machine-learning model algorithms. The accuracy of the models is measured using held-out testing data. 'Random' accuracy illustrates the accuracy of a meaningless model $(\sim 50 \%)$ and 'perfect' that of a model with no mistakes (100\%). All tests were more accurate than random (see other measures of model performance in Extended Data Fig. 9d-f and Supplementary Table 5c). c, Top 20 most-important features from RF models and GBM models. A rank product approach was used to determine overall top predictive features from both models. High values for variable importance indicate most influential predictors.

of $\mathrm{CpG}$ content is related to changes in $\mathrm{CpG}$ methylation regulation, RNA-seq revealed significant downregulation with organismal aging of DNA-methyltransferase encoding Dnmt1, demethylationcatalyzing TET encoding Tet 1 and Tet2, as well as methyl-CpG binding domain proteins encoding $M b d 4$ and $M b d 5$ (FDR < $5 \%$; Supplementary Table 1a). The presence of putative age-related changes in DNAme is consistent with the notion that DNAme patterns can serve as 'aging clocks' ${ }^{\text {'4 }}$.

Key predictors of age-related expression changes also included whether a gene was a target of specific TFs: Stat5a, Mtf2, Sirt6 and Foxo1 (Fig. 5c and Supplementary Table 5b). Although causality cannot be inferred from machine learning, predictors provide a list of candidate drivers/mediators of programs related to organismal aging. Only a subset of top TF predictors were themselves differentially regulated with aging (such as Stat5a, Mtf2 and Nod2; Extended Data Fig. 9g and Supplementary Table 1a). Thus, it is possible that the activity of TF predictors without RNA-level regulation may occur through post-translational regulation. Notably, Stat5a mediates the effects of granulocyte-macrophage colony-stimulating factor (GM-CSF) on granulocyte differentiation ${ }^{75}$ and is crucial for mature neutrophil survival ${ }^{76}$. Although the role of Mtf2 in neutrophils has not been studied, Mtf2 interacts with Jarid2 (another top predictor) to promote repressive $\mathrm{H} 3 \mathrm{~K} 27$ methylation ${ }^{77}$, which mediates recruitment to unmethylated $\mathrm{CpGs}^{78}$. Nod2 is a patternrecognition receptor that recognizes muramyl dipeptide, the minimal common motif of bacterial peptidoglycans ${ }^{79}$. Although Nod2 is not a TF, it has profound transcriptional effects on gene expression, with target genes including cytokines and chemokines ${ }^{80,81}$. Indeed, Nod2 is an important contributor of neutrophil-mediated innate immunity ${ }^{81}$. Sirt6 is a histone deacetylase tightly linked to the regulation of mammalian aging and longevity ${ }^{82,83}$. Sirt6 can limit inflammation by deacetylating the promoters of nuclear factor (NF)- $\mathrm{B}$ targets $^{84}$ and reducing cytokine production ${ }^{85}$. Consistently, Sirt6 knockout mice show liver inflammation with neutrophil infiltration $^{86}$. Thus, changes in activity for top predictive TFs may drive aspects of age-related transcriptional remodeling.

We next asked whether sex-dimorphic gene expression could be predicted from genomic information, local chromatin states and/or TF regulation (Fig. 6a). As an additional feature, we also included the chromosomal location of a gene (sex chromosomes versus autosomes). Our models assigned genes to the correct sex-based expression bias $>70 \%$ of the time on held-out testing data (Fig. $6 \mathrm{~b}$ and Supplementary Table 5c), consistent with the notion that sex-biased genes share a common regulatory signature. To understand which 
features were most predictive of male- or female-biased expression, we again examined predictor contribution from RF and GBM models (Fig. $6 \mathrm{c}$ and Supplementary Table 5d). Notably, being located on a sex chromosome (X or $\mathrm{Y}$ ) was a poor predictor of sex-dimorphic gene expression (166th combined importance rank; Supplementary Table 5d), consistent with the fact that genetic context was not a crucial driver (Extended Data Fig. 1a). Similar to our age-related models, the average promoter ATAC-seq signal and the fold change between females versus males in ATAC-seq signal were also top predictors for sex-biased gene expression (Fig. 6c and Supplementary Table $5 \mathrm{~d}$ ). The GC/CpG content of promoter and genes sequences also showed strong predictive power, consistent with the sex-dimorphic expression of DNAme regulators (male-biased expression for Dnmt1 and Tet3; Supplementary Table 1b).

Finally, we asked which TFs were most predictive of sex-dimorphic gene expression in neutrophils (Fig. $6 \mathrm{c}$ and Supplementary Table 5d). Being a known target of Foxm1, Sirt6, Mtf2 or Mybl2 was highly predictive of male versus female neutrophil-biased gene expression, suggesting that these may drive aspects of the sex-dimorphic neutrophil biology. Similar to above, only a small subset of top predictors from our models were themselves significantly regulated at the transcriptional level (for example Foxm1, Irf8; Extended Data Fig. 9h and Supplementary Table 1b). Although its role hasn't been explored in neutrophils, Foxm1 regulates the expression of cell cycle-related genes ${ }^{87}$ and is highly expressed in late committed neutrophil precursors ${ }^{40}$. The transcription factor Mybl2 also regulates cell cycle-related genes ${ }^{88}$ and can modulate differentiation and cell fate decision of myeloid progenitors ${ }^{89}$. Thus, top predictors of sex-dimorphic neutrophil gene expression Foxm1 and Mybl2 might be linked to observed sex-dimorphic expression of cell cycle and chromatin-related genes (Fig. 3 and Extended Data Fig. 7). Being a target of Sirt6 was also predictive of sex-dimorphic gene expression, in line with sex-dimorphic phenotypes of Sirt6 knockout and overexpression mice $^{82,83}$. In addition, consistent with sex dimorphism in lipid storage (Fig. 3f), Sirt6 is tightly linked to lipid metabolism ${ }^{90}$ and can limit lipid-droplet accumulation in foam cells ${ }^{91}$. In addition, Sirt6 activity can be directly regulated by FFAs (such as myristic, oleic and linoleic acid) ${ }^{92}$. Consistently, female neutrophils have higher levels of FFAs, including FFA(14:0) and FFA(18:2) (myristic and linoleic acid) and may thus have higher basal Sirt6 activity levels (Supplementary Table 1e,f). Thus, our machine-learning analysis reveals candidate regulators that may drive neutrophil sex dimorphism and will deserve further investigation.

Primary granule differences in female versus male neutrophils. Notably, when analyzing the top genes with the largest fold difference between male versus female neutrophils, we noticed that genes encoding primary neutrophil granule components showed top male-biased expression (such as Elane, Mpo, Prtn3 and Ctsg; Supplementary Table 1b), suggesting potential sex differences in granule loading. Thus, we investigated potential differences in granule biogenesis (Fig. 7a,b and Extended Data Fig. 10a-d). Neutrophils store a number of proteases and antimicrobial proteins in (1) primary/azurophilic granules, (2) secondary/specific granules and (3) tertiary/gelatinase granules. To unbiasedly assess whether neutrophil granule biology is sex dimorphic, we compiled a list of granule components from the literature (Supplementary Table 6). Consistent with our first observation, GSEA revealed a robust trend for increased RNA expression of primary granulerelated genes in male versus female neutrophils, regardless of age (Fig. 7a,b). In contrast, no significant enrichments were observed for genes encoding components of secondary or tertiary granules (FDR > 5\%; Extended Data Fig. 10a-d). Notably, we confirmed that protein levels of neutrophil elastase Elane were higher in young males versus young females by western blot $\left(P=3.9 \times 10^{-3}\right.$ in two-sided Wilcoxon's test between young females and male samples), although this sex difference disappeared in older animals (Extended Data Fig. 5d,e).

Neutrophil degranulation is a key aspect of the response of neutrophils to pathogens ${ }^{93}$ and mice without a functional copy of Elane have increased mortality upon sepsis ${ }^{94}$. However, excessive levels of circulating neutrophil elastase can be deleterious, amplifying septic shock ${ }^{95}$. Based on RNA-seq, we hypothesized that higher Elane expression in neutrophils could lead to increased Elane circulating levels, both in basal conditions and upon an immune challenge. To test this hypothesis, we obtained female and male young adult C57BL/6J mice and injected them with sterile PBS or lipopolysaccharide (LPS; major component of Gram-negative bacteria cell wall), for $1-6 \mathrm{~h}$, to mimic a septic state ${ }^{96}$ (Fig. 7c). Notably, males showed significantly higher levels of serum neutrophil elastase at 3 and $6 \mathrm{~h}$ after LPS exposure (Fig. $7 \mathrm{~d}$ ) and in the PBS-injected controls (Fig. 7d,e). Thus, young males had overall higher serum protein levels of Elane.

Finally, we then took advantage of a published proteomics dataset of human blood neutrophils from 68 healthy donors ${ }^{97}$. As the biological sex of human donors was not specified, we used the reported protein levels of DDX3Y, a protein encoded on the Y chromosome, as a proxy for the likelihood that the sample was derived from a male donor (higher levels of DDX3Y associated with males). Consistent with our mouse RNA-seq and serum ELISA, we found significant correlation between DDX3Y levels (likelihood of the sample coming from a male donor) and expression of primary granule proteins ELANE, MPO and CTSG, although not of PRTN3 (Extended Data Fig. 10e-g). Thus, our results suggest that neutrophils derived from human males have higher protein expression of key primary granule components. These observations suggest that, at least for these components, transcriptomic trends are predictive of protein-level trends and that the male bias in primary granule components observed in our mouse data may be conserved in humans.

\section{Discussion}

A resource for the study of neutrophils across sex and aging. We have generated transcriptomic, metabolomic, lipidomic and epigenomic datasets using bone-marrow neutrophils from young and old, female and male animals. This dataset is a large multiomic dataset for the study of neutrophils and includes both sexes and organismal aging, rather than focusing only on one sex or age group. Using this resource, we observed that (1) neutrophils are extremely sex dimorphic and that (2) organismal age leads to large scale transcriptomic and epigenomic remodeling of neutrophils. Of note, when analyzed separately, males had over tenfold more significantly regulated genes with age than females, suggesting that the molecular rate of neutrophil aging differs between sexes. Observed changes may likely be downstream of signals that drive aging itself, but these changes will impact immune responses in aged animals, participating in immune dysfunction.

We then predicted increased release of neutrophil elastase in males in control and sepsis-like conditions. Excess release of neutrophil elastase is known to exacerbate inflammation and cause tissue damage ${ }^{98}$. Thus, this resource should help open new avenues of research and identify candidate mediators that underlie sex differences in lifelong immunity. Future studies should investigate how the differences in bone marrow neutrophils are maintained, erased or amplified in circulating blood neutrophils.

Sex and aging influence neutrophil chromatin metabolism. We observed that regulation of chromatin metabolism is a hallmark of neutrophil aging and a key aspect with sex-dimorphic regulation. Far from being a mere regulatory layer for gene expression, chromatin organization has profound implications on NETosis ${ }^{8}$. Our analyses suggest that female neutrophils have an overall 'looser' chromatin, 

RNA expression of primary granule-associated genes

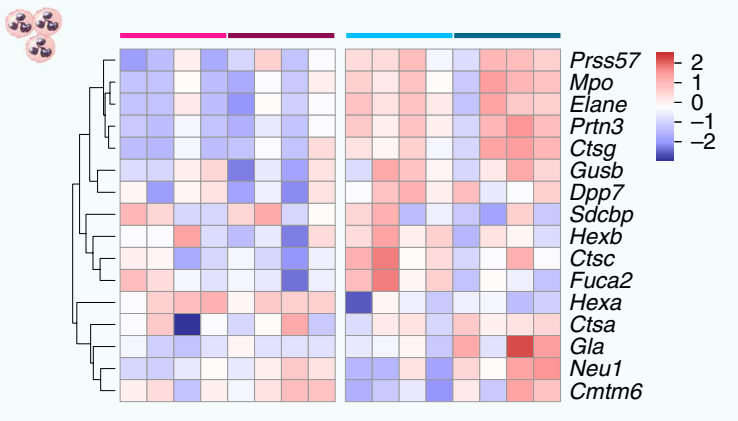
GSEA analysis of primary granule-associated genes

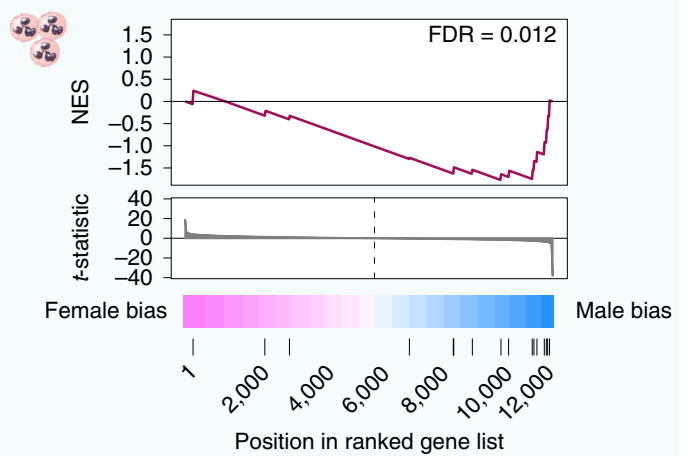

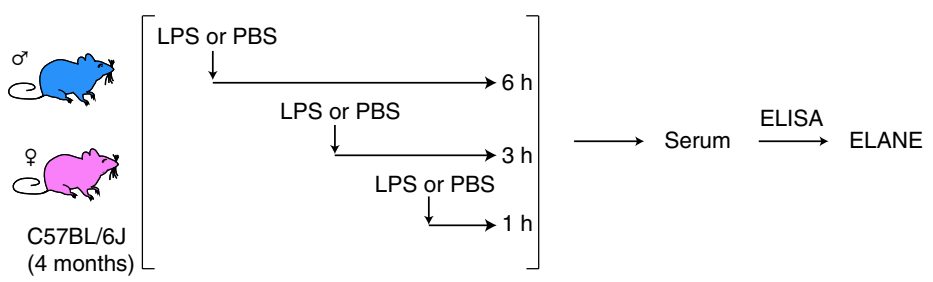

d

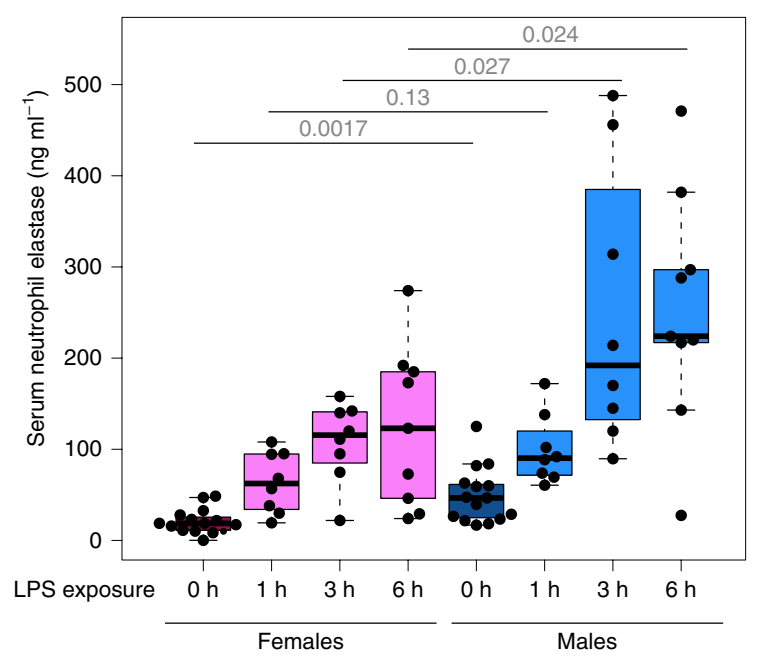

e Serum ELANE by ELISA (PBS)

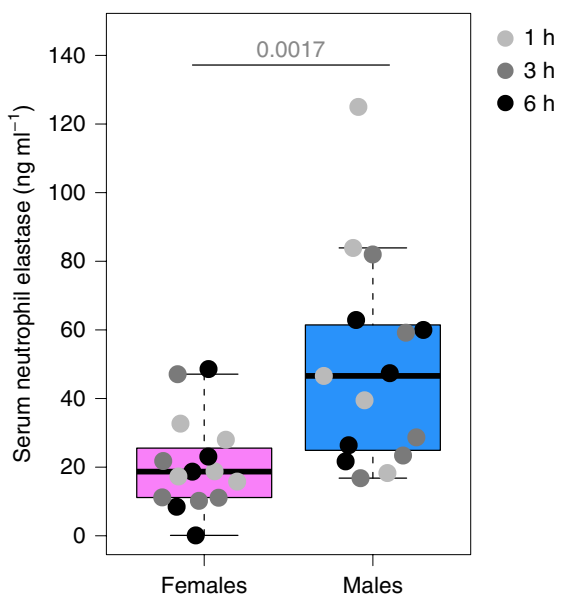

Fig. 7 | Male neutrophils express higher levels of primary granule genes, correlating with increased serum ELANE levels in control and septic mice. a, Heat map of normalized gene expression for primary (azurophilic) granule-related gene expression in our RNA-seq dataset. Also see Extended Data Fig. 10. b, GSEA of primary (azurophilic) granule-related gene expression reveals biased expression to male neutrophils. c, Setup scheme for serum ELANE measurement in control and sepsis-like mice. d,e, Analysis of ELANE protein levels in mouse serum by ELISA. Data are derived from three independent cohorts of young male versus female mice ( $n=5$ per sex and time point for 1,3 and $6 \mathrm{~h}$ PBS injection; $n=8$ per sex and time point for 1 and $3 \mathrm{~h}$ LPS and $n=9$ per sex and time point for 6 h LPS injection). For simplicity, all PBS-injected animals are reported as 'O h of LPS exposure' in $\mathbf{d}$ and are replotted with time-based color coding in e. Significance in nonparametric two-sided Wilcoxon rank-sum test. For box plots in d,e, the center line represents the sample median, the box limits consist of the 25 th and 75 th percentiles and the whiskers span $1.5 \times$ the interquartile range.

associated to the transcriptional upregulation of a number of transposable elements (TEs). Conversely (and regardless of sex), neutrophils from old individuals show increased chromatin condensation accompanied by reduced TE transcription. This contrasts with observation of age-related TE derepression in other contexts $^{19}$ and may reflect the unique neutrophil biology. Thus, it will be important to elucidate the mechanism driving differences in NETosis between sexes and throughout lifespan, with implications in aberrant chronic age-related inflammation ${ }^{10}$.

Machine learning as a powerful candidate-identification tool. By using machine learning, we showed that both age regulation and sex dimorphism in gene expression can be predicted accurately. The high accuracy of our models supports the notion of coordinated differences between (1) genes induced $v s$. repressed during aging and (2) genes expressed in a sex-dimorphic manner. Among important predictors of transcriptional states, we identified regulators whose activity change during organismal aging may underlie 'omic' profile remodeling throughout life (such as Foxo1 and Sirt6). We also identified putative mediators of sex-biased gene expression of neutrophils (such as Foxm1 and Mybl2). Although machine learning does not provide information about causality, predictors represent candidate mediators that could help shape neutrophil landscapes with aging or as a function of sex. Thus, it will be important to elucidate the potential role of these predictors to understand the emergence of age-related and sex-dimorphic phenotypes. 
Neutrophils as mediators for sex differences in immune aging. Accumulating evidence has shown that, even outside of reproduction, mammalian biology is extremely sex dimorphic ${ }^{99}$. This is especially relevant to aging, with large sex differences in baseline lifespan and in response to pro-longevity interventions ${ }^{58}$. Although this has not been broadly investigated with aging, pioneering studies have started to compare male and female cells, revealing profound sex dimorphism in gene regulation ${ }^{21,22}$. Consistently, accumulating evidence has shown that immune responses differ between sexes ${ }^{100,101}$.

Sex dimorphism in immunity is exemplified in the current health crisis, with men representing the majority of severe cases and deaths from COVID-19 (ref. ${ }^{102}$ ). Notably, NETs may drive aspects of inflammatory disease, lead to tissue damage ${ }^{8,30}$ and contribute to severe COVID-19 (ref. ${ }^{103}$ ). In addition, neutrophil dysfunction has been linked to the pathogenesis of a number of chronic diseases (such as macular degeneration ${ }^{104}$, stroke ${ }^{105}$, obstructive pulmonary disease ${ }^{106}$, atherosclerosis ${ }^{107}$ and cancer ${ }^{108}$ ). Thus, sex differences in neutrophils could constitute targets to optimize immune responses throughout life and help tailor therapeutics to men and women.

\section{Methods}

Mouse husbandry. All animals were treated and housed in accordance with the Guide for Care and Use of Laboratory Animals. All experimental procedures were approved by the University of Southern California’s Institutional Animal Care and Use Committee (IACUC) and are in accordance with institutional and national guidelines. Samples were derived from animals on approved IACUC protocol nos. 20770, 20804 and 21004.

For 'omics' analyses, male and female C57BL/6Nia mice (4-5- and 20-24-month-old animals) were obtained from the National Institute on Aging (NIA) colony at Charles Rivers. For the sepsis assays in young animals, male and female C57BL/6J mice (3-4-month-old animals) were obtained from Jackson Laboratories. Both Charles Rivers and Jackson Laboratories have specific pathogen-free facilities. Animals were acclimated at the specific-pathogen-free animal facility at University of Southern California (USC) for 2-4 weeks before any processing. The facility is on a 12-h light/dark cycle and animal housing rooms are maintained at $72^{\circ} \mathrm{F}$ and $30 \%$ humidity. All animals were euthanized between 8-11 am for 'omics' experiments, flow cytometry and NETosis assays. For the sepsis model experiments, animals were injected in the morning and euthanized between 4-6 pm. In all cases, animals were euthanized using a 'snaking order' across all groups to minimize batch-processing confounds due to circadian processes (including potential confounds due to neutrophil cellular 'age'). All animals were euthanized by $\mathrm{CO}_{2}$ asphyxiation followed by cervical dislocation.

Isolation of primary neutrophils from mouse bone marrow. The long bones of each mouse were collected and kept on ice in D-PBS (Corning) supplemented with $1 \%$ penicillin/streptomycin (Corning) until further processing. Muscle tissue was removed from the bones and bone marrow from cleaned bones was collected into clean tubes ${ }^{109}$. Red blood cells from the marrow were removed using Red Blood Cell Lysis buffer (Miltenyi Biotec, no. 130-094-183), according to the manufacturer's instructions, albeit with no vortexing step to avoid unscheduled neutrophil activation. The suspension was filtered on $70-\mu \mathrm{m}$ mesh filters (Miltenyi Biotec, no. 130-110-916) to retain only single cells for downstream processing. Neutrophils were isolated from other bone-marrow cells using MACS (Milteny Biotec, no. 130-097-658). Viability and yield were assessed using trypan blue exclusion and an automated COUNTESS cell counter (Thermo Fisher Scientific). Purified cells were pelleted at $300 \mathrm{~g}$ and snap-frozen in liquid nitrogen until processing for RNA, lipid or metabolite isolation.

Neutrophil purity estimates by flow cytometry. We used aging C57BL/6Nia mice from two independent cohorts to estimate potential differences in neutrophil purity with respect to sex and age. After an Fc-blocking step (Miltenyi Biotec, no. 130-092-575), MACS-purified neutrophils were then stained using APC-Ly6G (Invitrogen, no. 17-9668-80) and Vioblue-Cd11b (Miltenyi Biotec, no. 130-113238 ) at a 1:50 dilution according to the manufacturer's instructions. Stained cells were then analyzed by flow cytometry on a MACS Quant Analyzer 10 (Miltenyi Biotec). Flow cytometry results were processed using the FlowLogic v.7 software. Purity of MACS-isolated bone-marrow neutrophils in young and aged male and female animals are reported in Extended Data Fig. 3. Raw cytometry data were deposited on Figshare (https://doi.org/10.6084/m9.figshare.14043932.v1).

Purified neutrophil heterogeneity estimate by flow cytometry. We used flow cytometry analysis to evaluate the heterogeneity/maturity of our isolated neutrophils from two independent cohorts of aging C57BL/6Nia mice. We used a panel of antibodies designed to evaluate population heterogeneity of cells purified by MACS based on defined subpopulations of neutrophils in the literature ${ }^{110-112}$ (see below). Specifically, among live cell singlets, proneutrophils were defined as c-Kit ${ }^{+} \mathrm{Ly} 6 \mathrm{~g}^{-} \mathrm{Cd} 81^{+}$, with subpopulations of proneutrophil 1 defined as $\mathrm{c}-\mathrm{Kit}^{+} \mathrm{Ly} 6 \mathrm{G}^{-} \mathrm{Cd} 81^{+} \mathrm{CD} 11 \mathrm{~b}^{-} \mathrm{CD} 106^{-}$and pro-neutrophil 2 as c-Kit ${ }^{+} \mathrm{Ly} 6 \mathrm{G}^{-} \mathrm{Cd} 81^{+} \mathrm{CD} 11 \mathrm{~b}^{+} \mathrm{CD} 106^{+}$. Pre-neutrophils were defined as $\mathrm{Cd} 11 \mathrm{~b}^{+} \mathrm{Ly} 6 \mathrm{G}^{+} \mathrm{c}-\mathrm{Kit}^{+} \mathrm{Cxcr} 4^{+}$cells. Immature neutrophils were defined as

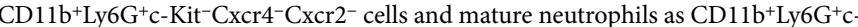
$\mathrm{Kit}^{-} \mathrm{Cxcr} 4^{-} \mathrm{Cxcr} 2^{+}$. Fresh versus 'aged' subsets of mature neutrophils were defined respectively as $\mathrm{CD} 11 \mathrm{~b}^{+} \mathrm{Ly}_{6 \mathrm{G}}{ }^{+} \mathrm{c}-\mathrm{Kit}^{-} \mathrm{Cxcr} 4^{-} \mathrm{Cxcr} 2^{+} \mathrm{CD} 62 \mathrm{~L}^{+}$versus $\mathrm{CD} 11 \mathrm{~b}^{+} \mathrm{Ly}_{6 \mathrm{G}}{ }^{+} \mathrm{c}-\mathrm{Kit}^{-} \mathrm{Cxcr} 4^{-} \mathrm{Cxcr} 2^{+} \mathrm{CD}^{-} 2 \mathrm{~L}^{-}$cells.

Before analyzing compositional heterogeneity on MACS-purified neutrophils, to account for spill-over from different lasers, compensation was performed using appropriate compensation beads (Miltenyi Biotec, no. 130-104-693 for Miltenyi Biotec antibodies; Thermo Fisher Scientific, no. 01-3333-42 for others). The compensation file was saved and loaded before analyzing neutrophils. Compensation was rerun anytime at least one fresh vial of antibody had to be used for the staining experiment.

Antibodies used for this heterogeneity panel were:

\section{- CD184 (CXCR4)-PE-Vio770 (clone REA107; Miltenyi Biotec, 130-102-914) at} 1:10 dilution

- CD81-PE (clone EAT2; Miltenyi Biotec, 130-102-632) at 1:10 dilution

- CD11b-Vioblue (clone REA592; Miltenyi Biotec, 130-113-810) at 1:50 dilution

- Ly6G-PerCP-Vio700 (clone REA526; Miltenyi Biotec, 130-117-500) at 1:50 dilution

- CD182 (CXCR2)-APC-Vio770 (clone REA942; Miltenyi Biotec, 130-115-637) at 1:50 dilution

- CD62L-FITC (clone REA828; Miltenyi Biotec, 130-112-835) at 1:50 dilution

- CD106 (VCAM-1)-APC (clone REA971; Miltenyi Biotec, 130-116-324) at 1:50 dilution

- CD117 (c-kit)-Brilliant Violet 510 (clone ACK2; BioLegend, 135119) at 1:20 dilution

After isolation by MACS, $2.5 \times 10^{5}$ cells were washed once by adding $1 \mathrm{ml}$ of PBS/EDTA 0.1\% BSA (MACS resuspension buffer) in a 5 -ml polystyrene roundbottom tube (Falcon, no. 352054), then centrifuged $300 \mathrm{~g}$ for $10 \mathrm{~min}$ at $4^{\circ} \mathrm{C}$. After an Fc-blocking step (Miltenyi Biotec, no. 130-092-575), MACS-purified neutrophils were then stained with antibodies for $20 \mathrm{~min}$ at $4{ }^{\circ} \mathrm{C}$ and excess antibody was washed away using resuspension buffer. Stained cells were then analyzed by flow cytometry on a MACS Quant Analyzer 10 (Miltenyi Biotec) and flow cytometry data were analyzed using FlowLogic v.7. Gating was determined using fluorescence-minus-one controls for each color used in the experiment to ensure that positive populations were solely associated with the antibody for that specific marker (Extended Data Fig. 4a). Due to the low amount of $\mathrm{c}-\mathrm{kit}^{+}$events in MACS-purified neutrophils, we also ran whole bone marrow (pooled by group in one cohort) with the same scheme to confirm that positive labeling by this antibody occurs normally on bone-marrow cells before purification (Extended Data Fig. 4b). Both sets of raw cytometry data, including fluorescence-minus-one controls and compensation files, were deposited to Figshare (https://doi.org/10.6084/m9.figshare.14043929.v1, https://doi. org/10.6084/m9.figshare.14043938.v1).

Estimate of neutrophil proportion in bone marrow in young female and male mice. We used aging C57BL/6Nia mice from two independent cohorts to estimate potential differences in neutrophil proportions within nucleated bone-marrow cells with respect to sex and age. An aliquot of bone-marrow cell suspension was obtained after bone-marrow extraction, red blood cell lysis and filtration on 70- $\mu \mathrm{m}$ mesh filters (see above). Cell composition analysis was obtained using the Hemavet 950FS at the USC Leonard Davis School of Gerontology mouse phenotyping core. Percentage of cells was used instead of absolute cell numbers to avoid confounding results due to animal size.

Evaluation of cell-cycle patterns of MACS-purified neutrophils by flow cytometry. We used aging C57BL/6Nia mice from two independent cohorts to estimate cell cycle proportions of MACS-purified neutrophils with respect to sex and age. We utilized a standard method using DNA content, as measured by propidium iodide staining of fixed cells, to estimate the phases of the cell cycle (G0/G1, S and G2/M). For each biological sample, $5 \times 10^{5}$ cells were aliquoted and fixed with $70 \%$ ethanol at $-20^{\circ} \mathrm{C}$ overnight. The next day, cells were washed with DPBS (Corning) and nuclear DNA was stained for $30 \mathrm{~min}$ at room temperature in labeling buffer ( $50 \mu \mathrm{g} \mathrm{ml}^{-1}$ propidium iodide (Alfa Aesar), $100 \mu \mathrm{g} \mathrm{ml}^{-1} \mathrm{RNaseA}$ (Invitrogen), $0.05 \%$ Triton X-100 (Sigma) in DPBS (Corning)). Stained cells were then analyzed by flow cytometry on a MACS Quant Analyzer 10 (Miltenyi Biotec) and flow cytometry data were analyzed using FlowLogic v.7. Raw cytometry data were deposited to Figshare (https://doi.org/10.6084/m9.figshare.14043926.v1).

RNA purification and RNA-seq library preparation. For RNA isolation, frozen cell pellets were resuspended in $1 \mathrm{ml}$ of Trizol reagent (Thermo Fisher Scientific) and total RNA was purified following the manufacturer's instructions. 
RNA quality was assessed using the Agilent Bioanalyzer platform at the USC Genome Core using the RNA integrity number. Then, $500 \mathrm{ng}$ of total RNA was subjected to ribosomal-RNA depletion using the NEBNext rRNA Depletion kit (New England Biolabs), according to the manufacturer's protocol. Strand specific RNA-seq libraries were then constructed using the SMARTer Stranded RNA-seq kit (Clontech), according to the manufacturer's protocol. Libraries were quality controlled on the Agilent Bioanalyzer 2100 platform at the USC Genome Core before multiplexing the libraries for sequencing. Paired-end 75-bp reads were generated on the Illumina NextSeq500 platform at the SC2 Core at CHLA (original cohort) or paired-end 150-bp reads were generated on the Illumina HiSeq-Xten platform at the Novogene Corporation (replicate cohort).

RNA-seq analysis pipeline. To best mimic the first RNA-seq cohort, paired-end 150 -bp reads from the replicate cohort of neutrophil RNA-seq were hard-trimmed to 75 bp using Fastx Trimmer. Both sets of paired-end reads were processed using Trimgalore v.0.4.4 (github.com/FelixKrueger/TrimGalore) (1) to retain only high quality bases with phred score $>15$ and (2) to eliminate biases due to priming by hard clipping the first six bases of each read. Only pairs with both reads retaining a length of $>45 \mathrm{bp}$ after trimming were retained for further processing. Trimmed reads were mapped to the mm 10 genome reference using STAR v.2.5.0 ${ }^{113}$. Read counts were assigned to genes from the UCSC mm10 reference using subread v.1.5.3 (ref. ${ }^{114}$ ) and were imported into R to perform differential gene expression analysis. Based on general RNA-seq processing guidelines, only genes with mapped reads in at least 6 out of 16 RNA-seq libraries were considered to be expressed and retained for downstream analysis. Due to high sample-to-sample variability, we used surrogate variable analysis to estimate and correct for unwanted experimental noise ${ }^{115}$. R package 'sva' v.3.34 was used to estimate surrogate variables and the removeBatchEffect function from 'limma' v.3.42.2 was used to regress out the effects of surrogate variables and RNA integrity differences (RNA integrity number scores) from raw read counts. The 'DESeq2' R package (DESeq2 v.1.26.0) was used for further processing of the RNA-seq data in $\mathrm{R}^{116}$. Notably, sex was encoded as a categorical variable (female versus male) and age was encoded as a continuous numerical variable. Genes with FDR $<5 \%$ were considered statistically significant and are reported in Supplementary Table 1a,b.

Dimensionality reduction for exploratory data analysis. To perform MDS analysis ${ }^{117}$, we used a distance metric between samples based on the Spearman's rank correlation value (1-Rho), which was then provided to the core R command 'cmdscale'. Dimensionality reduction was applied to pre-normalized data, as described in relevant sections.

Replicate and public neutrophil RNA-seq comparison. To assess the robustness of transcriptional changes linked to biological sex and/or organismal age, we used (1) a small replicate RNA-seq cohort of primary bone-marrow neutrophils with all four conditions, (2) a dataset from mouse spleen neutrophils from young adult female versus males from the ImmGen consortium (6-week-old animals; GEO datasets GSE124829) 22 and (3) a dataset from human blood neutrophils (ages unknown; GEO Datasets GSE145231) ${ }^{24}$ (Extended Data Fig. 2a,b). Reads were mapped to mm 10 and hg38, respectively using STAR and reads were summarized to genes using subread as before. DESeq2-normalized fold changes were then used to estimate differential gene expression as a function of age and of sex. For mouse datasets, the comparison directly assessed the DESeq2-normalized fold changes for genes with FDR $<5 \%$ in the original cohort RNA-seq. For the human dataset, orthologs of significant mouse genes in humans were identified using the R 'biomaRt' v.2.42.1 package and DESeq2-normalized fold changes were then compared.

Putative protein-protein interaction network analysis. Genes with significant regulation according to our DEseq2 analysis (FDR $<5 \%)$ were used as input for network analysis with NetworkAnalyst v.3.0 (ref. ${ }^{118}$ ). For age-related gene regulation, significant genes and DEseq 2 calculated $\log _{2}$ (fold change) were used as input for a single network analysis. For sex-dimorphic gene regulation, female- and male-biased gene lists were used as separate inputs for the analysis. To avoid network clusters due to sex-chromosome-encoded genes, only significant autosomal genes were included in the sex-dimorphism gene expression networks In both cases, NetworkAnalyst was set up to use PPI information derived from IMEx/InnateDB data, a knowledgebase specifically geared for analyses of innate immune networks ${ }^{119}$, to construct putative PPI networks. In each case, the largest subnetwork determined by NetworkAnalyst was used in figures and analyses.

Functional enrichment analysis (transcriptomics). We used the GSEA paradigm ${ }^{120}$ through the 'phenotest' v.1.34.0 R package. GO term annotations were obtained from ENSEMBL through Biomart (Ensembl 99) and gene-term association with only author statement support (GO evidence codes 'TAS' and 'NAS') or unclear evidence (GO evidence code 'ND') were filtered out. Other annotations were obtained from the Molecular Signature Database v.7.0 (Reactome and KEGG) ${ }^{120,121}$ and the Harmonizome database (ChEA, JASPAR, ENCODE, GEO TF targets) ${ }^{122}$. To improve target coverage, we summarized putative TF targets, including FOXO TF targets compiled in our previous study ${ }^{18}$, to have a reduced, unique and nonredundant list of TF targets summarized from all these sources. The DEseq $2 t$-statistic was used to generate the ranked list of genes for functional enrichment analysis, both for the sex and aging effects. For ease of reading, only the top ten most-significant pathways with negative NES and top ten most-significant pathways with positive NES are shown in figures if more than that passed the FDR $<5 \%$ significance threshold. All significant gene sets are reported in Supplementary Table 3 (aging) and Supplementary Table 4 (sex). In parallel, functional enrichment was also independently assessed using the ingenuity pathway analysis portal, using genes with a significant male or female bias $(F D R<5 \%)$

Western blot analysis of Padi4 and Elane protein levels. To prepare neutrophil lysates, $\sim 5$ million cells were collected from aging C57BL/6Nia mice from two independent cohorts ( $n=9$ animals per group). Flash-frozen cell pellets were resuspended in $200 \mu \mathrm{l}$ ice-cold lysis buffer ( $10 \mathrm{mM}$ Tris- $\mathrm{HCl}$ (pH 8.0), 1\% SDS, $1 \times$ Halt Protease and Phosphatase Inhibitor Cocktail (Life Technologies, no. 78442)) and incubated on ice for $30 \mathrm{~min}$. Samples were sonicated (Thermo Fisher Scientific, no. FB120; $60 \%$ power, $30 \mathrm{~s} \mathrm{ON} / 120 \mathrm{~s}$ OFF, four cycles) and centrifuged at $16,000 \mathrm{~g}$ for $20 \mathrm{~min}$ at $4^{\circ} \mathrm{C}$. Supernatant was collected and boiled in $4 \times$ Laemmli Sample Buffer (Bio-Rad, no. 1610747). Proteins were separated on 10\% SDS-PAGE gels and wet-transferred onto PVDF membranes (GE Healthcare, no. 10600021). To assess loading and transfer efficiency, membranes were stained using the Ponceau S solution (Sigma, P7170) for $2 \mathrm{~min}$ at room temperature. Membranes were blocked using $5 \%$ milk (Carnation) in $1 \times$ TBST buffer (TBS (Alfa Aesar J62938), supplemented with $0.05 \%$ Tween-20 (Bio-Rad no. 161-0781)) for $1 \mathrm{~h}$ at room temperature. Membranes were incubated with primary antibodies diluted in 5\% milk in TBST (anti- $\beta$-actin: Cell Signaling D6A8 at 1:5,000 dilution; antiELANE: Abcam ab68672 at 1:1,000 dilution; and anti-PADI4: Abcam, ab96758 at $1: 3,000$ dilution) for $16 \mathrm{~h}$ at $4^{\circ} \mathrm{C}$. After three 5 -min washes using $1 \times \mathrm{TBST}$ buffer, membranes were incubated with an HRP-conjugated secondary antibody (goat anti-rabbit IgG H\&L, Abcam, ab205718 at 1:10,000 dilution) for $1 \mathrm{~h}$ at room temperature. For visualization, membranes were treated with chemiluminescence solution according to manufacturer's manual (Bio-Rad, no. 1705062 and Thermo Fisher Scientific, no. 34580) and images were taken using Azure Biosystems c280.

For band intensity quantification, ImageJ (v.1.53) was used. Grayscale converted images were imported to ImageJ and intensity was measured from all bands. Background intensity was subtracted from each measurement. Anti- $\beta$ actin-relative values from each membrane were normalized by the median value of the specific membrane to mitigate membrane-specific variations.

All raw original images and cropped equivalents, as well as ImageJ quantification, have been made available on Figshare (https://doi.org/10.6084/ m9.figshare.14154665.v1) and as a source data file for Extended Data Fig. 5.

Chemicals for LC-MS. LC-MS-grade solvents and mobile-phase modifiers were obtained from Thermo Fisher Scientific (water, acetonitrile, methanol, methyl tertbutyl ether (MTBE)) and Sigma-Aldrich (ammonium acetate).

Metabolite and lipid extraction from neutrophils. Metabolites and lipids were extracted from neutrophil cell pellets and analyzed in a randomized order. Extraction was performed using a biphasic separation protocol with ice-cold methanol, MTBE and water ${ }^{123}$. Briefly, $300 \mu \mathrm{l}$ of methanol spiked-in with 54 deuterated internal standards provided with the Lipidyzer platform (SCIEX, cat. no. 5040156, LPISTDKIT-101) was added to the cell pellet, samples were vigorously vortexed for $20 \mathrm{~s}$ and sonicated in a water bath three times for $30 \mathrm{~s}$ on ice. Lipids were solubilized by adding $1,000 \mu \mathrm{l}$ of MTBE and incubated under agitation for $1 \mathrm{~h}$ at $4{ }^{\circ} \mathrm{C}$. After addition of $250 \mu \mathrm{l}$ of ice-cold water, the samples were vortexed for $1 \mathrm{~min}$ and centrifuged at $14,000 \mathrm{~g}$ for $5 \mathrm{~min}$ at $20^{\circ} \mathrm{C}$. The upper phase containing the lipids was then collected and dried down under nitrogen. The dry lipid extracts were reconstituted with $300 \mu \mathrm{l}$ of $10 \mathrm{mM}$ ammonium acetate in 9:1 methanol:toluene for analysis. The lower phase containing metabolites was subjected to further protein precipitation by adding four times of ice-cold 1:1:1 isopropanol:acetonitrile:water spiked in with 17 labeled internal standards and incubating for $2 \mathrm{~h}$ at $-20^{\circ} \mathrm{C}$. The supernatant was dried down to completion under nitrogen and resuspended in $100 \mu \mathrm{l}$ of 1:1 methanol:water for analysis.

Untargeted LC-MS metabolomics. Data acquisition. Metabolic extracts were analyzed four times using hydrophilic liquid chromatography (HILIC) and reversephase liquid chromatography (RPLC) separation in both positive and negative ionization modes as previously described ${ }^{124}$. Data were acquired on a Thermo Q Exactive plus mass spectrometer equipped with a HESI-II probe and operated in full MS scan mode. MS/MS data were acquired on pool samples consisting of an equimolar mixture of all the samples in the study. HILIC experiments were performed using a ZIC-HILIC column $2.1 \times 100 \mathrm{~mm}, 3.5 \mu \mathrm{m}, 200 \AA$ (Merck Millipore) and mobile-phase solvents consisting of $10 \mathrm{mM}$ ammonium acetate in 50:50 acetonitrile:water (A) and $10 \mathrm{mM}$ ammonium acetate in 95:5 acetonitrile:water (B). RPLC experiments were performed using a Zorbax SBaq column $2.1 \times 50 \mathrm{~mm}, 1.7 \mu \mathrm{m}, 100 \AA$ (Agilent Technologies) and mobile-phase solvents consisting of $0.06 \%$ acetic acid in water $(\mathrm{A})$ and $0.06 \%$ acetic acid in methanol (B). Data quality was ensured by (1) injecting 6- and 12-pool samples 
to equilibrate the LC-MS system before running the sequence for RPLC and HILIC, respectively, (2) sample randomization for metabolite extraction and data acquisition and (3) checking mass accuracy, retention time and peak shape of internal standards in every samples.

Data processing. Data from each mode were independently analyzed using Progenesis QI software v.2.3 (Nonlinear Dynamics). Metabolic features from blanks and that didn't show sufficient linearity upon dilution were discarded. Only metabolic features present in $>33 \%$ of the samples in each group were kept for further analysis and missing values were imputed by drawing from a random distribution of small values in the corresponding sample ${ }^{125}$.

Metabolic feature annotation. Annotation confidence levels for each metabolite were provided following the Metabolomics Standards Initiative (MSI) confidence scheme. Peak annotation was first performed by matching experimental $\mathrm{m} / \mathrm{z}$, retention time and MS/MS spectra to an in-house library of analytical-grade standards (level 1). Remaining peaks were identified by matching experimental $\mathrm{m} / \mathrm{z}$ and fragmentation spectra to publicly available databases including the Human Metabolome Database (HMDB) (http://www.hmdb.ca/), MassBank of North America (MoNA) (http://mona.fiehnlab.ucdavis.edu/) and MassBank (http://www.massbank.jp/) using the R package 'MetID' (v.0.2.0) ${ }^{126}$ (level 2). Briefly, metabolic feature tables from Progenesis QI were matched to fragmentation spectra with an $m / z$ and a retention time window of $\pm 15 \mathrm{ppm}$ and $\pm 30 \mathrm{~s}$ (HILIC) and \pm 20 s (RPLC), respectively. When multiple MS/MS spectra matched a single metabolic feature, all matched MS/MS spectra were used for the identification. Next, MS1 and MS2 pairs were searched against public databases and a similarity score was calculated using the forward dot-product algorithm, which takes into account both fragments and intensities. Metabolites were reported if the similarity score was $>0.4$. Level 3 corresponds to unknown metabolites.

Targeted lipidomics with the Lipidyzer platform. Data acquisition. Lipid extracts were analyzed using the Lipidyzer platform that comprises a 5500 QTRAP System equipped with a SelexION differential mobility spectrometry interface (SCIEX) and a high flow LC-30AD solvent delivery unit (Shimazdu). A full description of the method is available elsewhere ${ }^{123}$. Briefly, the lipid molecular species were identified and quantified using multiple reaction monitoring (MRM) and positive/negative switching. Two acquisition methods were employed covering ten lipid classes; method 1 had SelexION voltages turned on, whereas method 2 had SelexION voltages turned off. Lipidyzer data were reported by the Lipidomics Workflow Manager (LWM) v.1.0.5.0 software, which calculates concentration in $\mathrm{nmol} \mathrm{g}^{-1}$ for each detected lipid as average intensity of the analyte MRM/average intensity of the most structurally similar internal standard MRM multiplied by its concentration. Data quality was ensured by (1) tuning the differential mobility spectrometry compensation voltages using a set of lipid standards (SCIEX, no. 5040141) after each cleaning, more than $24 \mathrm{~h}$ of idling or $3 \mathrm{~d}$ of consecutive use, (2) performing a quick system suitability test (QSST) (SCIEX, no. 50407) before each batch to ensure acceptable limit of detection for each lipid class, (3) sample randomization for lipid extraction and data acquisition and (4) triplicate injection of lipids extracted from a reference plasma sample (SCIEX, no. 4386703) at the beginning of the batch.

Data preprocessing. Lipids detected in fewer than $66 \%$ of the samples in each group were discarded and missing values were imputed in each class by drawing from a random distribution of small values in the corresponding sample ${ }^{125}$.

Differential analysis of metabolomics and lipidomics data. Metabolomics and lipidomics datasets were first normalized to the total protein content as determined by BCA assay (Pierce, no. 23225) to account for differential starting material quantity. Then, variance stabilizing normalization was applied to the data using 'limma' v.3.42.2, as recommended by previous studies ${ }^{127,128}$. Differential analysis for metabolomic or lipidomic features was performed using 'limma' in R. Features with an FDR $<5 \%$ were considered to be statistically significant.

For analysis of the lipidomics data by lipid class (Supplementary Fig. 1d and Supplementary Table 1f), lipids were summarized by class after BCA and VSN corrections. To determine regulation at the class level, because of the small number of analyzed classes, a basic linear model approach was used through base R 'Im' function and FDR correction was applied using the base R 'p.adjust' function. Lipid classes with an FDR $<5 \%$ were considered statistically significant.

Functional enrichment analysis for metabolomics. As only one metabolite was significantly regulated with aging, we focused on analyzing differentially regulated metabolite sets with respect to sex. To analyze the directional regulation of metabolic pathways from untargeted metabolomics, we used R package 'MetaboAnalystR' v.2.0.4 (ref. ${ }^{129}$ ) to perform PSEA ${ }^{130}$ of KEGG metabolic pathways. Using the 'mummichog' method ${ }^{131}$, we provided an input table of metabolic peaks represented by mass over charge ratios $(\mathrm{m} / z)$ and retention time, limma-derived $P$ value and $t$-scores and analysis mode (negative or positive ion) to have maximum sensitivity for the functional enrichment analysis of untargeted metabolomic data. All significant gene sets $(\mathrm{FDR}<5 \%)$ are reported in Supplementary Table $4 \mathrm{f}$.
Integrated functional enrichment analysis for RNA-seq and metabolomics using IMPaLA. Based on the differential analyses results in RNA-seq and metabolomics, we focused on analyzing differentially regulated genes and metabolites with respect to sex. To provide an integrated view of RNA-seq and metabolomics results, we took advantage of the IMPaLA paradigm ${ }^{132}$. We used the IMPaLA v.12 web interface (http://impala.molgen.mpg.de/), which evaluates overrepresentation of genes and metabolites across 5,055 annotated pathways from 12 databases, with default parameters. For the joint analysis, we used genes with FDR $<5 \%$ with respect to biological sex, using gene symbols as input IDs. For metabolic features, we selected only the manually validated species (levels 1 and 2) with FDR $<5 \%$ with respect to biological sex. Finally, we also selected lipid species FDR $<5 \%$ with respect to biological sex. Importantly, we used HMDB IDs as input IDs for the metabolomic/lipidomic side of the analysis, thus analyzing only features with corresponding HMDB IDs. Joint analysis of significant features with a male bias, or, separately, those with female bias, were uploaded separately to the server. All pathways with an overall combined FDR $<5 \%$ are reported in Supplementary Table $4 \mathrm{~h}$.

Functional enrichment analysis for lipidomics. Since there was no significant difference in lipid composition with aging, we focused on analyzing differentially regulated lipid sets with respect to sex. The Lipid Ontology (LION) website was used to perform functional enrichment analysis of lipids ${ }^{133}$. Lipid features with FDR $<5 \%$ with a male or female bias were uploaded to the server. For ease of reading, only the ten most-significant pathways with male and with female bias are shown in Fig. 3f. All significant LION terms are reported in Supplementary Table $4 \mathrm{~g}$.

Neutrophil ATAC-seq library generation. An independent cohort of C57BL/6Nia mice was used to assess age- and sex-related differences in chromatin architecture using ATAC-seq. To assay potential differences in the chromatin landscape of mouse bone marrow neutrophils across age and sex, we used the omni-ATAC protocol starting from 50,000 MACS-purified cells ${ }^{134}$. Libraries were quality controlled on the Agilent Bioanalyzer 2100 platform at the USC Genome Core before pooling. Libraries were multiplexed and sequenced on the Illumina HiSeqXten platform as paired-end 150-bp reads at the Novogene Corporation.

ATAC-seq preprocessing pipeline. Paired-end ATAC-seq reads were adaptertrimmed using NGmerge 0.2 (ref. ${ }^{135}$ ), which clips overhanging reads in a sequence-independent fashion and has been recommended for use in ATAC-seq preprocessing. Trimmed paired-end were mapped to the mm10 genome build using bowtie 2 (v.2.2.6) ${ }^{136}$. After alignment, PCR duplicates were removed using the 'rmdup' function of samtools (v.1.5). To minimize analytical artifacts from uneven sequencing depth between biological samples/libraries, libraries were randomly downsampled to the same depth for downstream analyses using PicardTools (v.2.20.3) or samtools (v.1.5). We used the peak finding function of HOMER (v.4.11) to identify ATAC-seq accessible regions ${ }^{137}$.

Differential accessibility analysis with ATAC-seq. We extracted a normalized read count matrix from our downsampled bam files over merged HOMER regions using R package 'Diffbind' v.2.14 (ref. ${ }^{138}$ ). We used this extracted matrix for downstream analyses. Because of sample-to-sample variability, we used surrogate variable analysis ${ }^{115}$ to estimate and correct for experimental noise, similar to the RNA-seq analysis. R package 'sva' v.3.34 was used to estimate surrogate variables and the 'removeBatchEffect' function from 'limma' v.3.42.2 was used to regress out the effects of surrogate variables, PCR duplicate content and variation in reads mapping to peaks according to Diffbind (fraction of reads in peaks). The 'DESeq2' $\mathrm{R}$ package (v.1.26.0) was used for further processing of ATAC-seq data. Regions with FDR $<5 \%$ were considered statistically significant.

Functional enrichment analysis of differentially accessible regions from ATACseq. We used the significantly differentially accessible peaks identified by DESeq2 at $\mathrm{FDR}<5 \%$ to analyze functional enrichments linked to these regions. For this purpose, we leveraged the 'GREAT' v.4.0.4 tool $^{139}$, which is specifically optimized to identify functional enrichment starting from genomic regions. We used all ATACseq accessible regions as the background for enrichment. All other options were left as default parameters. Results were exported as 'tsv' and processed in R to filter significant regions at FDR $<5 \%$. Filtered results are reported in Supplementary Tables $3 \mathrm{f}$ and $4 \mathrm{i}$.

Chromatin architecture analysis from ATAC-seq datasets. To analyze the underlying chromatin architecture differences between neutrophils isolated from different biological groups, we used the NucleoATAC v.0.3.4 software ${ }^{68}$. As NucleoATAC requires high sequencing depth to reliably measure nucleosome profiles, we pooled depth-matched reads from each biological group to attain this depth as recommended by authors of the software ${ }^{68}$. We extracted similar metrics from the NucleoATAC output to what was described in previous studies using this software to investigate chromatin architecture ${ }^{140}$. All comparisons between groups were tested for statistical significance using a two-tailed Wilcoxon rank-sum test (nonparametric test). 
Repetitive element transcription analysis. To evaluate potential differential regulation of transposable elements, we used the 'analyzeRepeats.pl' functionality of the HOMER software to count STAR-mapped reads over repetitive elements ${ }^{137}$. To allow for whole-library normalization, reads mapping over transcripts were also counted using the same HOMER script. Read counts were imported into R to estimate differential repeat expression using the DESeq2 (v.1.26.0) R package ${ }^{116}$.

Neutrophil NETosis assay. We used a flow cytometry-based NETosis assay protocol using extracellularized DNA staining by SYTOX Green to quantify cells undergoing NETosis, adapted from a flow-cytometry-based protocol ${ }^{141}$ and a 96-well plate plate-reader protocol ${ }^{142}$, both with neutrophils in suspension. Briefly, MACS-purified neutrophils were resuspended in a concentration of $2 \times 10^{6}$ cells ml ${ }^{-1}$ in neutrophil culture medium (RPMI 1640 without phenol red (Hyclone), supplemented with $1 \%$ penicillin/streptomycin (Corning) and $0.1 \%$ BSA (Akron Biotechnology)). We then used $1 \times 10^{6}$ cells, aliquoted into sterile microcentrifuge tubes, one per starting biological sample. SYTOX green (Thermo Fisher Scientific, no. S7020) was added to each sample to a final concentration of $200 \mathrm{nM}$. Neutrophil medium supplemented with dimethylsulfoxide (DMSO) (vehicle) or 50 nM PMA (Sigma, no. P1565) was added to each tube. Tubes were slowly inverted three times to mix. Then, $2 \times 10^{5}$ cells were seeded in technical quadruplicates in wells of a sterile black 96-well plate (Greiner BioOne) and incubated in a humidified incubator with $5 \% \mathrm{CO}_{2}$ at $37^{\circ} \mathrm{C}$ for $2 \mathrm{~h}$. The fraction of cells positive for SYTOX Green in each well was quantified using the MACSQuant10 and analyzed using Flowlogic v.7. To account for differences in basal levels of NETosis across samples, NETosis was expressed as induction of NETosis as (median of PMA technical quadruplicates) / (median of DMSO technical quadruplicates). The raw cytometry dataset was deposited to Figshare (https://doi.org/10.6084/m9.figshare.14043923.v1).

TNF- $\alpha$-primed NETosis analysis. To compare our results with previously published results on NETosis in aged neutrophils ${ }^{13,14}$, which were obtained with direct TNF- $\alpha$ priming ${ }^{13}$ or in conditions known to mimic TNF- $\alpha$ priming ${ }^{14}$ (thioglycolate elicitation of peritoneal neutrophils ${ }^{71}$ ), we also performed NETosis analysis in primed conditions, using a slightly modified protocol from above to include a priming step. Specifically, MACS-purified neutrophils cells were resuspended in neutrophil culture medium and first pre-warmed after isolation in a humidified incubator with $5 \% \mathrm{CO}_{2}$ at $37^{\circ} \mathrm{C}$ for $15 \mathrm{~min}$. Then, $2 \times 10^{6}$ cells were aliquoted into a sterile microcentrifuge tube and primed with $10 \mathrm{ng} \mathrm{ml}^{-1}$ mouse TNF- $\alpha$ (PeProTech, no. 315-01 A) in a humidified incubator with $5 \% \mathrm{CO}_{2}$ at $37^{\circ} \mathrm{C}$ for $15 \mathrm{~min}$. Cells were further aliquoted, supplemented with SYTOX green, DMSO/ PMA, incubated and analyzed as above. The raw cytometry dataset was deposited to Figshare (https://doi.org/10.6084/m9.figshare.14043923.v1).

Feature extraction for machine-learning analysis. For each significantly age-regulated or sex-dimorphic gene in neutrophils according to DEseq2 $($ FDR $<5 \%)$, we extracted a number of features associated with that gene. First, we took advantage of our ATAC-seq data to evaluate the chromatin architecture of neutrophils (see above). For each gene, we extracted (1) the average promoter accessibility by ATAC-seq in fragments per kilobase of transcript per million mapped reads across all four conditions (promoters were defined as the region between -500 bp to +150 bp with regard to annotated transcriptional start sites in mm10 build according to HOMER), as well as (2) the average $\log _{2}$ (fold change) in accessibility between old and young neutrophils (age-regulation models) or the average $\log _{2}$ (fold change) in accessibility between female and male neutrophils (sex-dimorphism models). Second, we took advantage of gene sets coverage known of predicted targets of transcriptions factors: ChEA, ENCODE, JASPAR and GEO TF perturbation information from the Harmonizome database ${ }^{122}$ and transcriptional targets of FOXO transcription factors from GEO experiments compiled in our previous study ${ }^{18}$. To reduce extraneous features, we engineered a TF target feature following these steps: (1) TF targets were summarized from all putative sources, (2) only TFs with evidence of expression in primary neutrophils according to RNA-seq were retained as features and (3) only TFs with at least 25 targets across significant age-related genes (age-regulation models) or sex-dimorphic genes (sexdimorphism models) were retained. This process yielded 357 (age-regulation models) and 249 (sex-dimorphism models) TF target sets used as features for model training. Finally, we included several DNA sequence features for each gene: the percentage of CG nucleotide and the percentage of CpG dinucleotide in promoters and exons, computed using HOMER. To note, HOMER was only able to provide information for 3,421 of 3,653 genes with significant age regulation and 1,636 of 1,734 genes with significant sex-dimorphic regulation. Finally, to determine whether location of the gene on autosomes versus chromosomes was a key predictive factor for sex-dimorphic gene expression, we also included, for the sex-dimorphic gene expression models, a feature encoding whether the gene is located on autosomes or sex chromosomes (X or Y).

Machine-learning analysis for age-regulated or sex-dimorphic genes. We trained machine-learning classification models for two questions: (1) models for ageregulated gene expression and (2) models for sex-dimorphic gene expression.
For (1), age-regulation machine-learning models were built to learn whether up- or downregulated genes could be discriminated using genomic features and predict potential master regulators. For (2), sex-dimorphism machine-learning models were built to learn whether female and male-biased genes could be discriminated using genomic features and predict potential master regulators.

In both cases, we built classification models using seven classification algorithms as implemented through R package 'caret' (v.6.0-86). Auxiliary R packages were used with caret to implement neural networks (v.7.3-13), RFs (v.4.614), GBMs (v.2.1.5), radial kernel support vector machines (v.0.9-29), sparse linear discriminant analysis (v.0.1-9), conditional inference trees ('party', v.1.3-4) and regularized logistic regression ('LiblineaR', v.2.10-8). Caret was allowed to optimize final model parameters on training data using tenfold cross validation. Accuracies, sensitivities, specificities and area under the curve (using package 'pROC',

v.1.16.2) for all trained classifiers were estimated using a test set of randomly held-out one-third of the data (not used in the training phase) obtained using the 'createDataPartition' function. Feature importance estimation was only conducted using tree-based RF and GBM methods, as other algorithms do not natively allow for it. The feature importance was computed and scaled by caret for the RF and GBM models.

Reanalysis of DIA proteomics data from human neutrophils. We obtained dataindependent acquisition (DIA) proteomics results from the supplemental material of a recent study ${ }^{97}$. We used normalized expression values from the article's supplemental material and used reported 'donor-specific Spectronaut protein expression values' for further analysis. To exclude potential confounders linked to disease, we used only data from 68 healthy donors and excluded data from patients with disease. As the article did not report the biological sex of donors, we used detected expression levels of DDX3Y, a Y-chromosome-encoded protein, as a proxy for the likelihood of the sample belonging to a male donor. We then examined rank correlation statistics between protein expression of DDX3Y compared to that of human orthologs of top male-biased primary granules genes from our mouse RNA-seq data (ELANE, MPO, PRTN3 and CTSG). The significance of the Spearman rank correlation is reported.

Mouse sepsis model through intraperitoneal LPS injection. Young adult (3-4-month-old) C57BL/6J mice were exposed to LPS, a pathogen-associated molecular pattern found in the cell wall of Gram-negative bacteria, to elicit a sepsis-like response ${ }^{96}$. Briefly, mice were injected intraperitoneally with $2 \mu \mathrm{g}$ of LPS per gram of body weight, using a sterile LPS stock (Sigma, no. L5293) diluted in PBS and control animals were injected with sterile $\mathrm{PBS}^{96}$. Animals were monitored hourly for up to $6 \mathrm{~h}$ after injection for the occurrence and severity of endotoxemia ${ }^{96}$. We did not observe gross differences in the state of female versus male animals injected with LPS over the course of the experiment, although it is possible that such differences would have emerged at longer time points. After killing by $\mathrm{CO}_{2}$ asphyxiation and cervical dislocation at 1,3 and $6 \mathrm{~h}$ after injection, blood was collected from the heart. A total of 80 animals across three independent experimental days were used ( $n=8$ animals per sex, age and time group for LPS injections; $n=5$ animals per sex, age and time group for control PBS injections). To obtain serum for downstream analysis, the blood was left to clot for $\geq 1 \mathrm{~h}$ at room temperature in MiniCollect Serum SeparatorTubes (Greiner Bio-One). Serum was then separated from the clot using centrifugation at $2,000 \mathrm{~g}$ for $10 \mathrm{~min}$ and frozen at $-80^{\circ} \mathrm{C}$ until further use.

Quantification of serum neutrophil elastase (ELANE) by ELISA. Quantitative evaluation of circulating ELANE levels was performed from serum samples. ELISA quantification of serum ELANE levels was performed using Abcam's Mouse Neutrophil Elastase SimpleStep ELISA kit (ab252356) according to the manufacturer's instructions. Technical replicates from the same sample were averaged as one value before statistical analysis and plotting.

Statistics and reproducibility. All statistical analyses were conducted using $\mathrm{R}$ v.3.6.0-3.6.3. For all 'omic' analyses, results were corrected for multiple testing by the use of FDR significance correction. All statistical analyses were performed as specified in figure legends and in the corresponding methods section. We used nonparametric statistical tests whenever possible to avoid assuming normality of data distributions. No statistical methods were used to predetermine sample size, since effect sizes and variance were not known a priori. No data passing quality control checks were excluded from the analyses. All 'omic' samples were performed on independent biological samples, usually from one cohort of animals to minimize known issues linked to batch effects in such analyses ${ }^{143}$. As one exception, RNA-seq of bone marrow neutrophils was independently replicated on a smaller cohort, as neutrophils are known to be RNA-poor, which could add more than usual biological variability (Extended Data Fig. 2). With the exception of a test of the heterogeneity flow cytometry panel on bone-marrow cells (used to validate c-kit staining; Extended Data Fig. 4b and Supplementary Table 2b), all other experiments were performed on animals from at least two independent cohorts.

For each experiment, animals were processed in an alternating/snaking order rather than in large homogeneous groups to minimize group, batch and circadian effects. For observational aging studies, no randomization was possible as groups 
were biologically determined. For LPS injection experiments, animals were randomly allocated to the PBS or LPS group for time point of euthanasia, on a cage-level basis (animals from the same cage were randomly attributed to LPS or PBS injections; for each cohort, co-housed animals were used for the same time point; cages were randomly attributed to a specific time point for euthanasia). This process was performed independently for each of the three cohorts for LPS-induced endotoxemia experiments. Blinding was not relevant to the studies conducted as data were collected by automated systems (sequencing, MS, flow cytometry) or were in numeric form (ImageJ quantification of western blot bands, ELISA absorbance values), which cannot be influenced by subjectivity from the experimenter.

Reporting Summary. Further information on research design is available in the Nature Research Reporting Summary linked to this article.

\section{Data availability}

Sequencing data have been submitted to the Sequence Read Archive accessible through BioProject PRJNA630663. Untargeted metabolomics data were uploaded to metabolomics workbench DataTrack ID 2089. The lipidomics data are available as Supplementary Table 7 and were deposited in Figshare (https://doi.org/10.6084/ m9.figshare.14524278). Raw flow cytometry data were deposited to Figshare (https://doi.org/10.6084/m9.figshare.14043938.v1, https://doi.org/10.6084/ m9.figshare.14043932.v1, https://doi.org/10.6084/m9.figshare.14043929.v1, https://doi.org/10.6084/m9.figshare.14043926.v1 and https://doi.org/10.6084/ m9.figshare.14043923.v1). Western blot raw and cropped images have been deposited to Figshare (https://doi.org/10.6084/m9.figshare.14154665). We reanalyzed publicly available neutrophil RNA-seq data from Gene Expression Omnibus datasets GSE1248296 (6-week-old mouse spleen neutrophil samples) and GSE145231 (human blood neutrophil samples) and human neutrophil DIA proteomics data (https://www.ncbi.nlm.nih.gov/pmc/articles/PMC6442368/ bin/141289_1_supp_251888_pjqfps.xlsx). Functional gene annotations were obtained from ENSEMBL Biomart (https://www.ensembl.org/biomart/martview/), the Molecular Signature Database (http://www.gsea-msigdb.org/gsea/msigdb/ collections.jsp) and Harmonizome (https://maayanlab.cloud/Harmonizome/ download). Mass spectrometry peaks were identified by matching experimental $m / z$ and fragmentation spectra to publicly available databases, including HMDB (http://www.hmdb.ca/), MoNA (http://mona.fiehnlab.ucdavis.edu/) and MassBank (http://www.massbank.jp/). Source data for Extended Data Fig. 5d,e are provided with the paper.

\section{Code availability}

The analytical code is available on the Benayoun Laboratory GitHub repository (https://github.com/BenayounLaboratory/Neutrophil_Omics_2020). All R code was run using $\mathrm{R}$ v.3.6.0-3.6.3.

Received: 19 July 2020; Accepted: 10 June 2021; Published online: 19 July 2021

\section{References}

1. Nah, E. H., Kim, S., Cho, S. \& Cho, H. I. Complete blood count reference intervals and patterns of changes across pediatric, adult, and geriatric ages in Korea. Ann. Lab. Med. 38, 503-511 (2018).

2. Furze, R. C. \& Rankin, S. M. Neutrophil mobilization and clearance in the bone marrow. Immunology 125, 281-288 (2008).

3. Shah, B., Burg, N. \& Pillinger, M. H. in Kelley and Firestein's Textbook of Rheumatology 10th edn (eds Firestein, G. S., Budd, R. C., Gabriel, S. E., McInnes, I. B. \& O’Dell, J. R.) Ch 11 (Elsevier, 2017).

4. Ballesteros, I. et al. Co-option of neutrophil fates by tissue environments. Cell 183, 1282-1297 (2020).

5. Lahoz-Beneytez, J. et al. Human neutrophil kinetics: modeling of stable isotope labeling data supports short blood neutrophil half-lives. Blood 127, 3431-3438 (2016)

6. Pillay, J. et al. In vivo labeling with $2 \mathrm{H}_{2} \mathrm{O}$ reveals a human neutrophil lifespan of 5.4 days. Blood 116, 625-627 (2010)

7. Zhang, D. et al. Neutrophil ageing is regulated by the microbiome. Nature 525, 528-532 (2015).

8. Sollberger, G., Tilley, D. O. \& Zychlinsky, A. Neutrophil extracellular traps: the biology of chromatin externalization. Dev. Cell 44, 542-553 (2018).

9. Soehnlein, O., Steffens, S., Hidalgo, A. \& Weber, C. Neutrophils as protagonists and targets in chronic inflammation. Nat. Rev. Immunol. 17, 248-261 (2017).

10. Franceschi, C. \& Campisi, J. Chronic inflammation (inflammaging) and its potential contribution to age-associated diseases. J. Gerontol. A Biol. Sci. Med. Sci. 69, S4-S9 (2014).

11. Lu, R. J., Wang, E. K. \& Benayoun, B. A. Functional genomics of inflamm-aging and immunosenescence. Brief. Funct. Genomics https://doi. org/10.1093/bfop/elab009 (2021).

12. Tseng, C. W. \& Liu, G. Y. Expanding roles of neutrophils in aging hosts. Curr. Opin. Immunol. 29, 43-48 (2014)
13. Hazeldine, J. et al. Impaired neutrophil extracellular trap formation: a novel defect in the innate immune system of aged individuals. Aging Cell 13, 690-698 (2014).

14. Tseng, C. W. et al. Innate immune dysfunctions in aged mice facilitate the systemic dissemination of methicillin-resistant S. aureus. PLOS ONE 7, e41454 (2012).

15. Sapey, E. et al. Phosphoinositide 3-kinase inhibition restores neutrophil accuracy in the elderly: toward targeted treatments for immunosenescence. Blood 123, 239-248 (2014).

16. Simmons, S. R., Bhalla, M., Herring, S. E., Tchalla, E. Y. I. \& Bou Ghanem, E. N. Older but not wiser: The age-driven changes in neutrophil responses during pulmonary infections. Infect. Immun. https://doi.org/10.1128/ IAI.00653-20 (2021)

17. McLaughlin, M. E., Kao, R., Liener, I. E. \& Hoidal, J. R. A quantitative in vitro assay of polymorphonuclear leukocyte migration through human amnion membrane utilizing 111in-oxine. J. Immunol. Methods 95, 89-98 (1986)

18. Benayoun, B. A. et al. Remodeling of epigenome and transcriptome landscapes with aging in mice reveals widespread induction of inflammatory responses. Genome Res. 29, 697-709 (2019).

19. Lai, R. W. et al. Multi-level remodeling of transcriptional landscapes in aging and longevity. BMB Rep. 52, 86-108 (2019).

20. Klein, S. L. \& Flanagan, K. L. Sex differences in immune responses. Nat. Rev. Immunol. 16, 626-638 (2016).

21. Marquez, E. J. et al. Sexual-dimorphism in human immune system aging. Nat. Commun. 11, 751 (2020).

22. Gal-Oz, S. T. et al. ImmGen report: sexual dimorphism in the immune system transcriptome. Nat. Commun. 10, 4295 (2019).

23. Markman, J. L. et al. Loss of testosterone impairs anti-tumor neutrophil function. Nat. Commun. 11, 1613 (2020).

24. Gupta, S. et al. Sex differences in neutrophil biology modulate response to type I interferons and immunometabolism. Proc. Natl Acad. Sci. USA 117, 16481-16491 (2020).

25. Frisch, B. J. et al. Aged marrow macrophages expand platelet-biased hematopoietic stem cells via interleukin-1B. JCI Insight https://doi. org/10.1172/jci.insight.124213 (2019).

26. Kolaczkowska, E. The older the faster: aged neutrophils in inflammation. Blood 128, 2280-2282 (2016).

27. Adrover, J. M., Nicolas-Avila, J. A. \& Hidalgo, A. Aging: a temporal dimension for neutrophils. Trends Immunol. 37, 334-345 (2016).

28. Chen, $\mathrm{X}$. et al. ATAC-see reveals the accessible genome by transposasemediated imaging and sequencing. Nat. Methods 13, 1013-1020 (2016).

29. Denholtz, M. et al. Upon microbial challenge, human neutrophils undergo rapid changes in nuclear architecture and chromatin folding to orchestrate an immediate inflammatory gene program. Genes Dev. 34, 149-165 (2020).

30. Papayannopoulos, V. Neutrophil extracellular traps in immunity and disease. Nat. Rev. Immunol. 18, 134-147 (2018).

31. Brinkmann, V. et al. Neutrophil extracellular traps kill bacteria. Science 303, 1532-1535 (2004)

32. Papayannopoulos, V., Metzler, K. D., Hakkim, A. \& Zychlinsky, A. Neutrophil elastase and myeloperoxidase regulate the formation of neutrophil extracellular traps. J. Cell Biol. 191, 677-691 (2010).

33. Amulic, B. et al. Cell-cycle proteins control production of neutrophil extracellular traps. Dev. Cell 43, 449-462 (2017).

34. Lopez-Otin, C., Blasco, M. A., Partridge, L., Serrano, M. \& Kroemer, G. The hallmarks of aging. Cell 153, 1194-1217 (2013).

35. Riffelmacher, T. et al. Autophagy-dependent generation of free fatty acids is critical for normal neutrophil differentiation. Immunity 47, 466-480 (2017)

36. Park, S. Y. et al. Autophagy primes neutrophils for neutrophil extracellular trap formation during sepsis. Am. J. Respir. Crit. Care Med. 196, 577-589 (2017).

37. Bhattacharya, A. et al. Autophagy is required for neutrophil-mediated inflammation. Cell Rep. 12, 1731-1739 (2015).

38. Mitxelena, J. et al. An E2F7-dependent transcriptional program modulates DNA damage repair and genomic stability. Nucleic Acids Res. 46, 4546-4559 (2018)

39. Yuan, R. et al. Cyclin F-dependent degradation of E2F7 is critical for DNA repair and G2-phase progression. EMBO J. 38, e101430 (2019).

40. Kim, M.-H. et al. A late-lineage murine neutrophil precursor population exhibits dynamic changes during demand-adapted granulopoiesis. Sci. Rep. 7, 39804-39804 (2017)

41. Brown, A. K. \& Webb, A. E. Regulation of FOXO factors in mammalian cells. Curr. Top. Dev. Biol. 127, 165-192 (2018).

42. Dong, G. et al. FOXO1 regulates bacteria-induced neutrophil activity. Front Immunol. 8, 1088 (2017).

43. Thiam, H. R. et al. NETosis proceeds by cytoskeleton and endomembrane disassembly and PAD4-mediated chromatin decondensation and nuclear envelope rupture. Proc. Natl Acad. Sci. USA 117, 7326 (2020).

44. Rohrbach, A. S., Slade, D. J., Thompson, P. R. \& Mowen, K. A. Activation of PAD4 in NET formation. Front. Immunol. 3, 360 (2012). 
45. Cuthbert, G. L. et al. Histone deimination antagonizes arginine methylation. Cell 118, 545-553 (2004).

46. Li, P. et al. Regulation of p53 target gene expression by peptidylarginine deiminase 4. Mol. Cell Biol. 28, 4745 (2008).

47. Denis, H. et al. Functional connection between deimination and deacetylation of histones. Mol. Cell Biol. 29, 4982-4993 (2009).

48. Christophorou, M. A. et al. Citrullination regulates pluripotency and histone H1 binding to chromatin. Nature 507, 104-108 (2014).

49. Hossain, D., Barbelanne, M. \& Tsang, W. Y. Requirement of NPHP5 in the hierarchical assembly of basal feet associated with basal bodies of primary cilia. Cell Mol. Life Sci. 77, 195-212 (2020).

50. Marquis, J. F. et al. Interferon regulatory factor 8 regulates pathways for antigen presentation in myeloid cells and during tuberculosis. PLoS Genet. 7, e1002097 (2011)

51. Yáñez, A., Ng, M. Y., Hassanzadeh-Kiabi, N. \& Goodridge, H. S. IRF8 acts in lineage-committed rather than oligopotent progenitors to control neutrophil vs monocyte production. Blood 125, 1452-1459 (2015)

52. Salem, S., Salem, D. \& Gros, P. Role of IRF8 in immune cells functions, protection against infections, and susceptibility to inflammatory diseases. Hum. Genet. 139, 707-721 (2020).

53. Wynn, T. A. Cellular and molecular mechanisms of fibrosis. J. Pathol. 214 199-210 (2008).

54. Gregory, A. D. et al. Neutrophil elastase promotes myofibroblast differentiation in lung fibrosis. J. Leukoc. Biol. 98, 143-152 (2015)

55. Muller, W. A. Transendothelial migration: unifying principles from the endothelial perspective. Immunol. Rev. 273, 61-75 (2016).

56. Oh, I. H. \& Reddy, E. P. The myb gene family in cell growth, differentiation and apoptosis. Oncogene 18, 3017-3033 (1999).

57. Penniman, C. M. et al. Loss of FoxOs in muscle reveals sex-based differences in insulin sensitivity but mitigates diet-induced obesity. Mol. Metab. 30, 203-220 (2019).

58. Austad, S. N. \& Bartke, A. Sex differences in longevity and in responses to anti-aging interventions: a mini-review. Gerontology 62, 40-46 (2015).

59. Baker, M. J., Pan, D. \& Welch, H. C. Small GTPases and their guaninenucleotide exchange factors and GTPase-activating proteins in neutrophil recruitment. Curr. Opin. Hematol. 23, 44-54 (2016).

60. Richer, B. C., Salei, N., Laskay, T. \& Seeger, K. Changes in neutrophil metabolism upon activation and aging. Inflammation 41, 710-721 (2018).

61. Eltzschig, H. K. et al. Endogenous adenosine produced during hypoxia attenuates neutrophil accumulation: coordination by extracellular nucleotide metabolism. Blood 104, 3986-3992 (2004).

62. Eltzschig, H. K. et al. ATP release from activated neutrophils occurs via connexin 43 and modulates adenosine-dependent endothelial cell function. Circ. Res. 99, 1100-1108 (2006).

63. Mondanelli, G., Iacono, A., Allegrucci, M., Puccetti, P. \& Grohmann, U. Immunoregulatory interplay between arginine and tryptophan metabolism in health and disease. Front. Immunol. 10, 1565 (2019).

64. Jarc, E. \& Petan, T. A twist of FATe: lipid droplets and inflammatory lipid mediators. Biochimie 169, 69-87 (2020).

65. Schlager, S. et al. Adipose triglyceride lipase acts on neutrophil lipid droplets to regulate substrate availability for lipid mediator synthesis. J. Leukoc. Biol. 98, 837-850 (2015).

66. Meana, C. et al. Lipin-1 integrates lipid synthesis with proinflammatory responses during TLR activation in macrophages. J. Immunol. 193, 4614 (2014).

67. Buenrostro, J. D., Giresi, P. G., Zaba, L. C., Chang, H. Y. \& Greenleaf, W. J. Transposition of native chromatin for fast and sensitive epigenomic profiling of open chromatin, DNA-binding proteins and nucleosome position. Nat. Methods 10, 1213-1218 (2013).

68. Schep, A. N. et al. Structured nucleosome fingerprints enable highresolution mapping of chromatin architecture within regulatory regions. Genome Res. 25, 1757-1770 (2015)

69. Janssen, A., Colmenares, S. U. \& Karpen, G. H. Heterochromatin: guardian of the genome. Annu. Rev. Cell Dev. Biol. 34, 265-288 (2018).

70. Neubert, E. et al. Chromatin swelling drives neutrophil extracellular trap release. Nat. Commun. 9, 3767 (2018).

71. Itou, T., Collins, L. V., Thoren, F. B., Dahlgren, C. \& Karlsson, A. Changes in activation states of murine polymorphonuclear leukocytes (PMN) during inflammation: a comparison of bone marrow and peritoneal exudate PMN. Clin. Vaccine Immunol. 13, 575-583 (2006).

72. Consortium, E. P. An integrated encyclopedia of DNA elements in the human genome. Nature 489, 57-74 (2012).

73. Rausch, C., Hastert, F. D. \& Cardoso, M. C. DNA modification readers and writers and their interplay. J. Mol. Biol. 432, 1731-1746 (2020).

74. Horvath, S. \& Raj, K. DNA methylation-based biomarkers and the epigenetic clock theory of ageing. Nat. Rev. Genet. 19, 371-384 (2018).

75. Feldman, G. M. et al. STAT5A-deficient mice demonstrate a defect in granulocyte-macrophage colony-stimulating factor-induced proliferation and gene expression. Blood 90, 1768-1776 (1997)
76. Kimura, A. et al. The transcription factors STAT5A/B regulate GM-CSFmediated granulopoiesis. Blood 114, 4721-4728 (2009).

77. Zhang, Z. et al. PRC2 complexes with JARID2, MTF2, and esPRC2p48 in ES cells to modulate ES cell pluripotency and somatic cell reprogramming. Stem Cells 29, 229-240 (2011).

78. Perino, M. et al. MTF2 recruits polycomb repressive complex 2 by helical-shape-selective DNA binding. Nat. Genet. 50, 1002-1010 (2018).

79. Girardin, S. E. et al. Nod2 is a general sensor of peptidoglycan through muramyl dipeptide (MDP) detection. J. Biol. Chem. 278, 8869-8872 (2003)

80. Billmann-Born, S. et al. Genome-wide expression profiling identifies an impairment of negative feedback signals in the Crohn's disease-associated NOD2 variant L1007fsinsC. J. Immunol. 186, 4027 (2011).

81. Jeong, Y. J. et al. Nod2 and Rip2 contribute to innate immune responses in mouse neutrophils. Immunology 143, 269-276 (2014).

82. Kanfi, Y. et al. The sirtuin SIRT6 regulates lifespan in male mice. Nature 483, 218-221 (2012).

83. Peshti, V. et al. Characterization of physiological defects in adult SIRT6 mice. PLoS ONE 12, e0176371 (2017).

84. Kawahara, T. L. et al. SIRT6 links histone H3 lysine 9 deacetylation to NF-кB-dependent gene expression and organismal life span. Cell 136, 62-74 (2009).

85. Lappas, M. Anti-inflammatory properties of sirtuin 6 in human umbilical vein endothelial cells. Mediators Inflamm. 2012, 597514 (2012).

86. Xiao, C. et al. Progression of chronic liver inflammation and fibrosis driven by activation of c-JUN signaling in Sirt6 mutant mice. J. Biol. Chem. 287, 41903-41913 (2012).

87. Chen, X. et al. The forkhead transcription factor FOXM1 controls cell cycle-dependent gene expression through an atypical chromatin binding mechanism. Mol. Cell Biol. 33, 227 (2013).

88. Zhan, M. et al. The B-MYB transcriptional network guides cell cycle progression and fate decisions to sustain self-renewal and the identity of pluripotent stem cells. PLoS ONE 7, e42350 (2012).

89. Baker, S. J. et al. B-myb is an essential regulator of hematopoietic stem cell and myeloid progenitor cell development. Proc. Natl Acad. Sci. USA 111, 3122-3127 (2014).

90. Jung, S. M. et al. Non-canonical mTORC2 signaling regulates brown adipocyte lipid catabolism through SIRT6-FoxO1. Mol. Cell 75, 807-822 (2019).

91. He, J. et al. SIRT6 reduces macrophage foam cell formation by inducing autophagy and cholesterol efflux under ox-LDL condition. FEBS J. 284, 1324-1337 (2017).

92. Feldman, J. L., Baeza, J. \& Denu, J. M. Activation of the protein deacetylase SIRT6 by long-chain fatty acids and widespread deacylation by mammalian sirtuins. J. Biol. Chem. 288, 31350-31356 (2013).

93. Lacy, P. Mechanisms of degranulation in neutrophils. Allergy Asthma Clin. Immunol. 2, 98-108 (2006).

94. Belaaouaj, A. et al. Mice lacking neutrophil elastase reveal impaired host defense against Gram-negative bacterial sepsis. Nat. Med. 4, 615-618 (1998)

95. Okeke, E. B. et al. Inhibition of neutrophil elastase prevents neutrophil extracellular trap formation and rescues mice from endotoxic shock. Biomaterials 238, 119836 (2020).

96. Raduolovic, K., Mak’Anyengo, R., Kaya, B., Steinert, A. \& Niess, J. H. Injections of lipopolysaccharide into mice to mimic entrance of microbialderived products after intestinal barrier breach. J. Vis. Exp. https://doi. org/10.3791/57610 (2018).

97. Grabowski, P. et al. Proteome analysis of human neutrophil granulocytes from patients with monogenic disease using data-independent acquisition. Mol. Cell Proteom. 18, 760 (2019).

98. Chua, F. \& Laurent, G. J. Neutrophil elastase: mediator of extracellular matrix destruction and accumulation. Proc. Am. Thorac. Soc. 3 424-427 (2006).

99. Sampathkumar, N. K. et al. Widespread sex dimorphism in aging and age-related diseases. Hum. Genet. 139, 333-356 (2020).

100. Kovats, S. Estrogen receptors regulate innate immune cells and signaling pathways. Cell Immunol. 294, 63-69 (2015)

101. Angele, M. K., Pratschke, S., Hubbard, W. J. \& Chaudry, I. H. Gender differences in sepsis: cardiovascular and immunological aspects. Virulence 5, 12-19 (2014).

102. Scully, E. P., Haverfield, J., Ursin, R. L., Tannenbaum, C. \& Klein, S. L. Considering how biological sex impacts immune responses and COVID-19 outcomes. Nat. Rev. Immunol. 20, 442-447 (2020).

103. Barnes, B. J. et al. Targeting potential drivers of COVID-19: Neutrophil extracellular traps. J. Exp. Med. 217, e20200652 (2020).

104. Ghosh, S. et al. Neutrophils homing into the retina trigger pathology in early age-related macular degeneration. Commun. Biol. 2, 348 (2019).

105. Roy-O'Reilly, M. A. et al. Aging exacerbates neutrophil pathogenicity in ischemic stroke. Aging 12, 436-461 (2020). 
106. Meijer, M., Rijkers, G. T. \& van Overveld, F. J. Neutrophils and emerging targets for treatment in chronic obstructive pulmonary disease. Expert Rev. Clin. Immunol. 9, 1055-1068 (2013).

107. Ionita, M. G. et al. High neutrophil numbers in human carotid atherosclerotic plaques are associated with characteristics of rupture-prone lesions. Arterioscler. Thromb. Vasc. Biol. 30, 1842-1848 (2010).

108. Treffers, L. W., Hiemstra, I. H., Kuijpers, T. W., van den Berg, T. K. \& Matlung, H. L. Neutrophils in cancer. Immunol. Rev. 273, 312-328 (2016).

109. Amend, S. R., Valkenburg, K. C. \& Pienta, K. J. Murine hind limb long bone dissection and bone marrow isolation. JoVE https://doi. org/10.3791/53936 (2016).

110. Kwok, I. et al. Combinatorial single-cell analyses of granulocyte-monocyte progenitor heterogeneity reveals an early uni-potent neutrophil progenitor. Immunity 53, 303-318 (2020).

111. Evrard, M. et al. Developmental analysis of bone marrow neutrophils reveals populations specialized in expansion, trafficking, and effector functions. Immunity 48, 364-379 (2018).

112. Adrover, J. M. et al. A neutrophil timer coordinates immune defense and vascular protection. Immunity 50, 390-402 (2019).

113. Dobin, A. et al. STAR: ultrafast universal RNA-seq aligner. Bioinformatics 29, 15-21 (2013)

114. Liao, Y., Smyth, G. K. \& Shi, W. featureCounts: an efficient general purpose program for assigning sequence reads to genomic features. Bioinformatics 30, 923-930 (2014).

115. Leek, J. T. \& Storey, J. D. Capturing heterogeneity in gene expression studies by surrogate variable analysis. PLoS Genet. 3, 1724-1735 (2007)

116. Love, M. I., Huber, W. \& Anders, S. Moderated estimation of fold change and dispersion for RNA-seq data with DESeq2. Genome Biol. 15, 550 (2014).

117. Chen, Y. \& Meltzer, P. S. Gene expression analysis via multidimensional scaling. Curr. Protoc. Bioinformatics https://doi.org/10.1002/0471250953. bi0711s10 (2005)

118. Zhou, G. et al. NetworkAnalyst 3.0: a visual analytics platform for comprehensive gene expression profiling and meta-analysis. Nucleic Acids Res. 47, W234-W241 (2019).

119. Breuer, K. et al. InnateDB: systems biology of innate immunity and beyond-recent updates and continuing curation. Nucleic Acids Res. 41, D1228-D1233 (2013).

120. Subramanian, A. et al. Gene set enrichment analysis: a knowledge-based approach for interpreting genome-wide expression profiles. Proc. Natl Acad. Sci. USA 102, 15545 (2005)

121. Liberzon, A. et al. Molecular signatures database (MSigDB) 3.0. Bioinformatics 27, 1739-1740 (2011).

122. Rouillard, A. D. et al. The Harmonizome: a collection of processed datasets gathered to serve and mine knowledge about genes and proteins. Database 2016, baw100 (2016).

123. Contrepois, K. et al. Cross-platform comparison of untargeted and targeted lipidomics approaches on aging mouse plasma. Sci. Rep. 8, 17747 (2018).

124. Contrepois, K., Jiang, L. \& Snyder, M. Optimized analytical procedures for the untargeted metabolomic profiling of human urine and plasma by combining hydrophilic interaction (HILIC) and reverse-phase liquid chromatography (RPLC)-mass spectrometry. Mol. Cell Proteomics 14, 1684-1695 (2015).

125. Tyanova, S. et al. The Perseus computational platform for comprehensive analysis of (prote)omics data. Nat. Methods 13, 731-740 (2016).

126. Shen, X. et al. Metabolic reaction network-based recursive metabolite annotation for untargeted metabolomics. Nat. Commun. 10, 1516 (2019).

127. Jauhiainen, A. et al. Normalization of metabolomics data with applications to correlation maps. Bioinformatics 30, 2155-2161 (2014).

128. $\mathrm{Li}, \mathrm{B}$. et al. Performance evaluation and online realization of data-driven normalization methods used in LC/MS-based untargeted metabolomics analysis. Sci. Rep. 6, 38881 (2016).

129. Chong, J. \& Xia, J. MetaboAnalystR: an R package for flexible and reproducible analysis of metabolomics data. Bioinformatics 34, 4313-4314 (2018).

130. Ried, J. S. et al. PSEA: phenotype set enrichment analysis-a new method for analysis of multiple phenotypes. Genet. Epidemiol. 36, 244-252 (2012).

131. Li, S. et al. Predicting network activity from high throughput metabolomics. PLoS Comput. Biol. 9, e1003123 (2013).

132. Kamburov, A., Cavill, R., Ebbels, T. M. D., Herwig, R. \& Keun, H. C. Integrated pathway-level analysis of transcriptomics and metabolomics data with IMPaLA. Bioinformatics 27, 2917-2918 (2011).

133. Molenaar, M. R. et al. LION/web: a web-based ontology enrichment tool for lipidomic data analysis. Gigascience https://doi.org/10.1093/gigascience/ giz061 (2019).

134. Corces, M. R. et al. An improved ATAC-seq protocol reduces background and enables interrogation of frozen tissues. Nat. Methods 14, 959-962 (2017).
135. Gaspar, J. M. NGmerge: merging paired-end reads via novel empiricallyderived models of sequencing errors. BMC Bioinf. 19, 536 (2018).

136. Langmead, B. \& Salzberg, S. L. Fast gapped-read alignment with Bowtie 2. Nat. Methods 9, 357-359 (2012).

137. Heinz, S. et al. Simple combinations of lineage-determining transcription factors prime cis-regulatory elements required for macrophage and B cell identities. Mol. Cell 38, 576-589 (2010).

138. Ross-Innes, C. S. et al. Differential oestrogen receptor binding is associated with clinical outcome in breast cancer. Nature 481, 389-393 (2012).

139. McLean, C. Y. et al. GREAT improves functional interpretation of cis-regulatory regions. Nat. Biotechnol. 28, 495-501 (2010).

140. King, H. W., Fursova, N. A., Blackledge, N. P. \& Klose, R. J. Polycomb repressive complex 1 shapes the nucleosome landscape but not accessibility at target genes. Genome Res. 28, 1494-1507 (2018).

141. Masuda, S. et al. Measurement of NET formation in vitro and in vivo by flow cytometry. Cytometry A 91, 822-829 (2017).

142. Carmona-Rivera, C. \& Kaplan, M. J. Induction and Quantification of NETosis. Curr. Protoc. Immunol. 115, 14.41.1-14.41.14 (2016).

143. Goh, W. W. B., Wang, W. \& Wong, L. Why batch effects matter in omics data, and how to avoid them. Trends Biotechnol. 35, 498-507 (2017).

\section{Acknowledgements}

We thank D. Campo and S. Patel at the University of Southern California (USC) genome core for assistance in next-generation sequencing library quality control on the Agilent Bioanalyzer platform; F. Li at the SC2 Core at CHLA for help with sequencing of transcriptomic libraries on the Illumina NextSeq550; E. Christensen from Miltenyi Biotec for help designing and optimizing the flow cytometry panel. We thank T. Morgan and G. Navarette from the USC Leonard Davis School of Gerontology mouse phenotyping core for assistance with CBC analyses on the Hemavet 950FS. We acknowledge the use of the HPC resource at USC for computational analyses. We thank C. Lee (USC), K. Yen (USC), S. P. Curran (USC), M. Vermulst (USC), H. Goodridge (Cedars-Sinai Medical Center) and E. Bou Ghanem (University at Buffalo) for helpful insights and feedback on our study. Finally, we thank laboratory members A. Adler, C. Boriboun, C. McGill and E. K. Wang for helpful discussions and feedback on the study. We apologize for any papers not cited. This work was supported by a Diana Jacobs Kalman/AFAR Scholarships for Research in the Biology of Aging (to R.J.L.), GCRLE2020 post-doctoral fellowship from the Global Consortium for Reproductive Longevity and Equality at the Buck Institute, made possible by the Bia-Echo Foundation (to M.K.), NIA T32 AG052374 and NSF graduate research fellowship DGE-1842487 (to J.I.B.) and NIA R00 AG049934, Pew Biomedical Scholar award no. 00034120, an innovator grant from the Rose Hills foundation and the Kathleen Gilmore Biology of Aging research award (to B.A.B). This work was also partially supported by the National Cancer Institute Cancer Center Support Grant P30 CA014089 through the use of shared resources. The authors acknowledge the Center for Advanced Research Computing at the USC for providing computing resources that have contributed to the research results reported within this publication (https://carc.usc.edu).

\section{Author contributions}

S.T. and B.A.B. designed the study. R.J.L., S.T., M.K., N.K. and B.A.B. isolated and/or processed neutrophil samples. R.J.L. and B.A.B. performed flow cytometry experiments to characterize neutrophil phenotypes. K.C. experimentally processed samples for metabolomic and lipidomic profiling and pre-processed resulting data. M.K. performed neutrophil protein extraction and western blotting experiments. K.C. and M.E. performed manual validation/identification of significant metabolites from untargeted metabolomics data. R.L. and B.A.B. conducted the 'sepsis-like' experiment. J.I.B performed HEMAVET quantifications of cell types on bone-marrow cell suspensions. B.A.B. performed data analyses and wrote the manuscript with input from all other authors. All authors edited and commented on the manuscript.

\section{Competing interests}

The authors declare no competing interests.

\section{Additional information}

Supplementary information The online version contains supplementary material available at https://doi.org/10.1038/s43587-021-00086-8.

Correspondence and requests for materials should be addressed to B.A.B.

Peer review information Nature Aging thanks Janet Lord, Hongbo Luo and the other, anonymous, reviewer(s) for their contribution to the peer review of this work.

Reprints and permissions information is available at www.nature.com/reprints. Publisher's note Springer Nature remains neutral with regard to jurisdictional claims in published maps and institutional affiliations.

(c) The Author(s), under exclusive licence to Springer Nature America, Inc. 2021 
a Number of features with significant sex- or age-differences in mouse BM neutrophils (FDR<5\%)

\begin{tabular}{|c|c|c|c|c|}
\hline & $\begin{array}{r}\mathrm{RN} \\
\text { All chromosomes }\end{array}$ & Autosomes & Metabolomics & Lipidomics \\
\hline $\begin{array}{l}\text { Aging regulation } \\
\text { - Upregulated } \\
\text { - Downregulated }\end{array}$ & $\begin{array}{l}1681 \\
1972\end{array}$ & & $\begin{array}{l}1 \\
0\end{array}$ & $\begin{array}{l}0 \\
0\end{array}$ \\
\hline $\begin{array}{l}\text { Sex-dimorphic regulation } \\
\text { - Female-biased } \\
\text { - Male-biased }\end{array}$ & $\begin{array}{c}1073 \\
661 \\
\end{array}$ & $\begin{array}{c}1033 \\
638 \\
\end{array}$ & $\begin{array}{l}134 \\
204\end{array}$ & $\begin{array}{l}70 \\
63 \\
\end{array}$ \\
\hline
\end{tabular}

b Xist RNA-seq expression

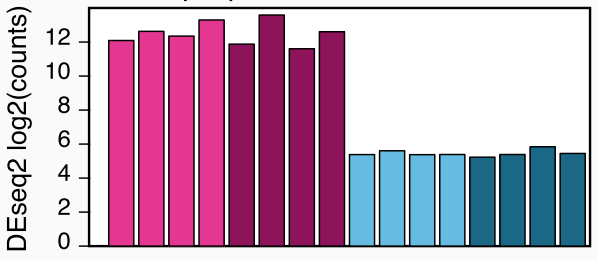

C Ddx3y RNA-seq expression

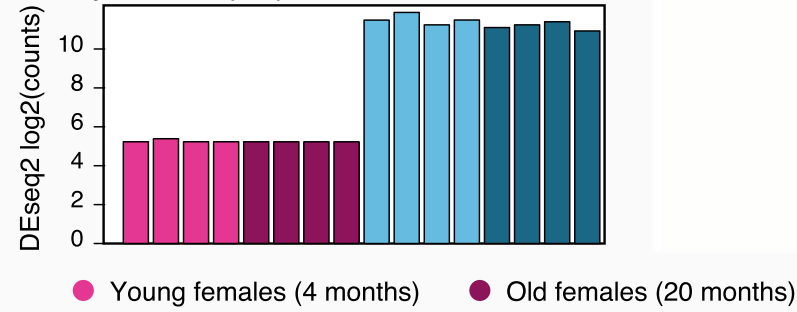

Position of genes with sex-dimorphic expression (FDR $<5 \%$ )

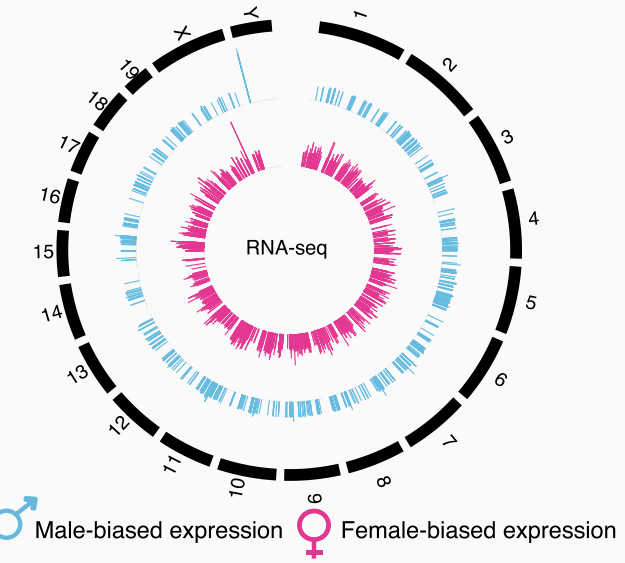

g Convergent age-related changes between sexes (FDR $<5 \%$ )

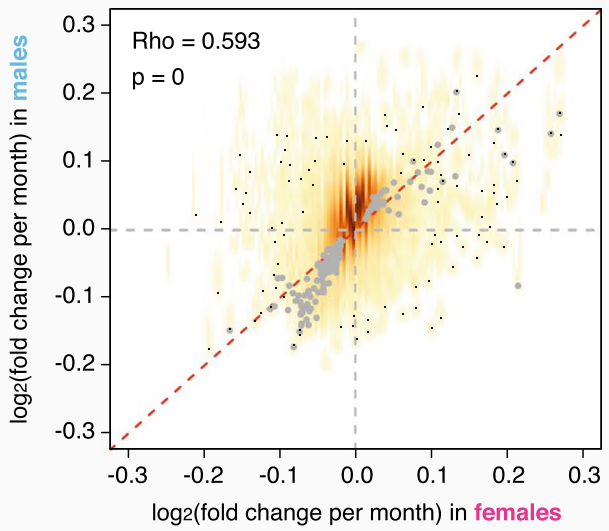

Extended Data Fig. 1 | See next page for caption. d Analysis of lipidomics data by lipid class

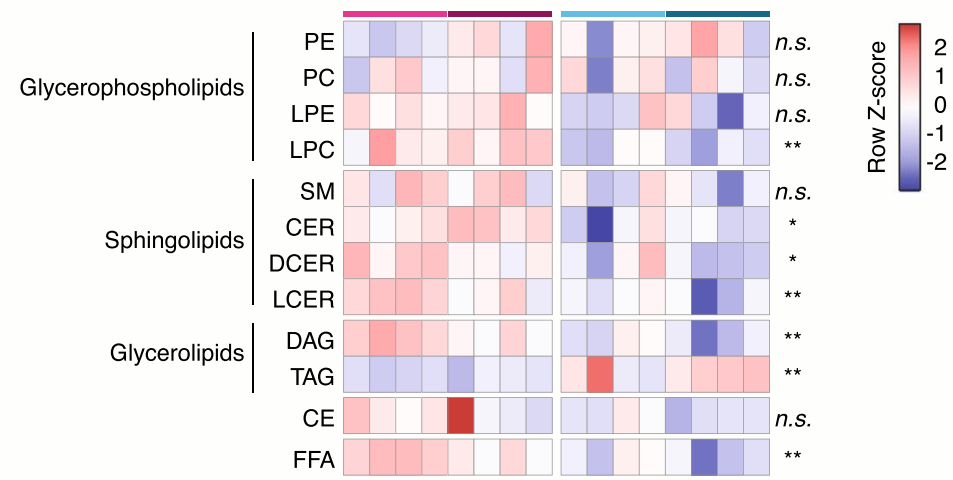

Young males (4 months) Old males (20 months) f Heatmap of age-regulated gene expression 3653 genes (FDR $<5 \%)$

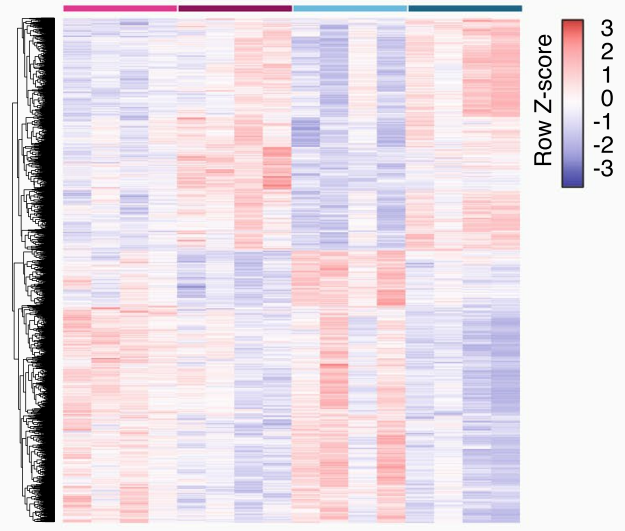

h Divergent age-related changes between sexes (FDR $<5 \%$ )

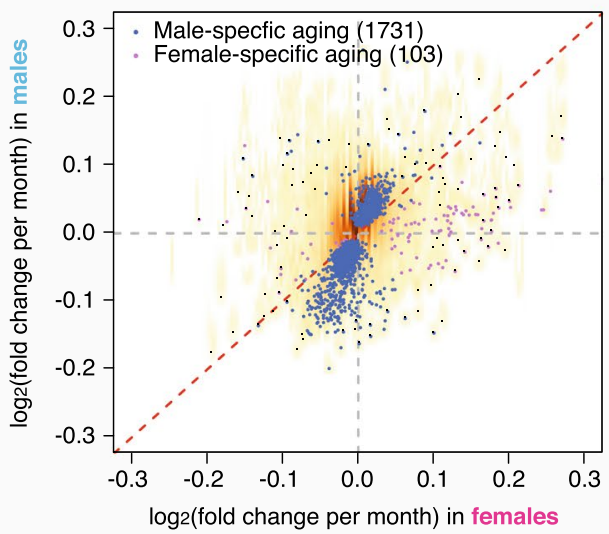


Extended Data Fig. 1 | A multi-omic analysis of primary mouse bone marrow neutrophils during aging and with respect to sex (continued). a, Table of significant 'omic' features as a function of organismal age and sex in our datasets based on DEseq2 (RNA-seq) or limma (Metabolomics and lipidomics), at FDR $<5 \%$. For the analysis of sex-dimorphism in gene expression, the number of significant genes located on autosomes (that is not on chromosomes $\mathrm{X}$ or $\mathrm{Y}$ ) is also reported. $\mathbf{b}$-c, Barplot of DESeq2-normalized log2 counts for Xist (b) and Ddx3y (c), showing the expected pattern between male and female samples, which acts as a quality check for our dataset. $\mathbf{d}$, Heatmap of lipidomic changes summarized by lipid classes. Significance of difference as a function of age or sex was evaluated using a linear model, and the significance of the sex coefficient (as reported by the R 'Im' function) is reported on the line of the heatmap. ${ }^{\star}: p<0.05 ;{ }^{\star *}: p<0.01 ; n . s .: p \geq 0.05$. See also Supplementary Table 1F. $\mathbf{e}$, Circular genome plot of the positions of genes with significant sex-biased gene expression in neutrophils (FDR $<5 \%$ ). $\mathbf{f}$, Heatmap of significant age-regulated genes (DESeq2 FDR $<5 \%$ ). $\mathbf{g}$-h, Correlation plot of age-related gene expression change according to DESeq2 in female vs. male neutrophils from RNA-seq, showing genes with (g) significant and concordant age-regulation in both sexes at FDR $5 \%$, or (h) genes with divergent age-regulation between sexes (FDR $<5 \%$ in one sex, and FDR $>15 \%$ in the other). Spearman Rank correlation (Rho), and significance of this correlation are reported in (g). Importantly, age was inputted into the DEseq2 model as a continuous numerical variable (expressed in months), which yields fold change values per month of life. 
a Fold-change in expression for genes with FDR $<5 \%$ in Cohort 1 with biological sex (RNA-seq)

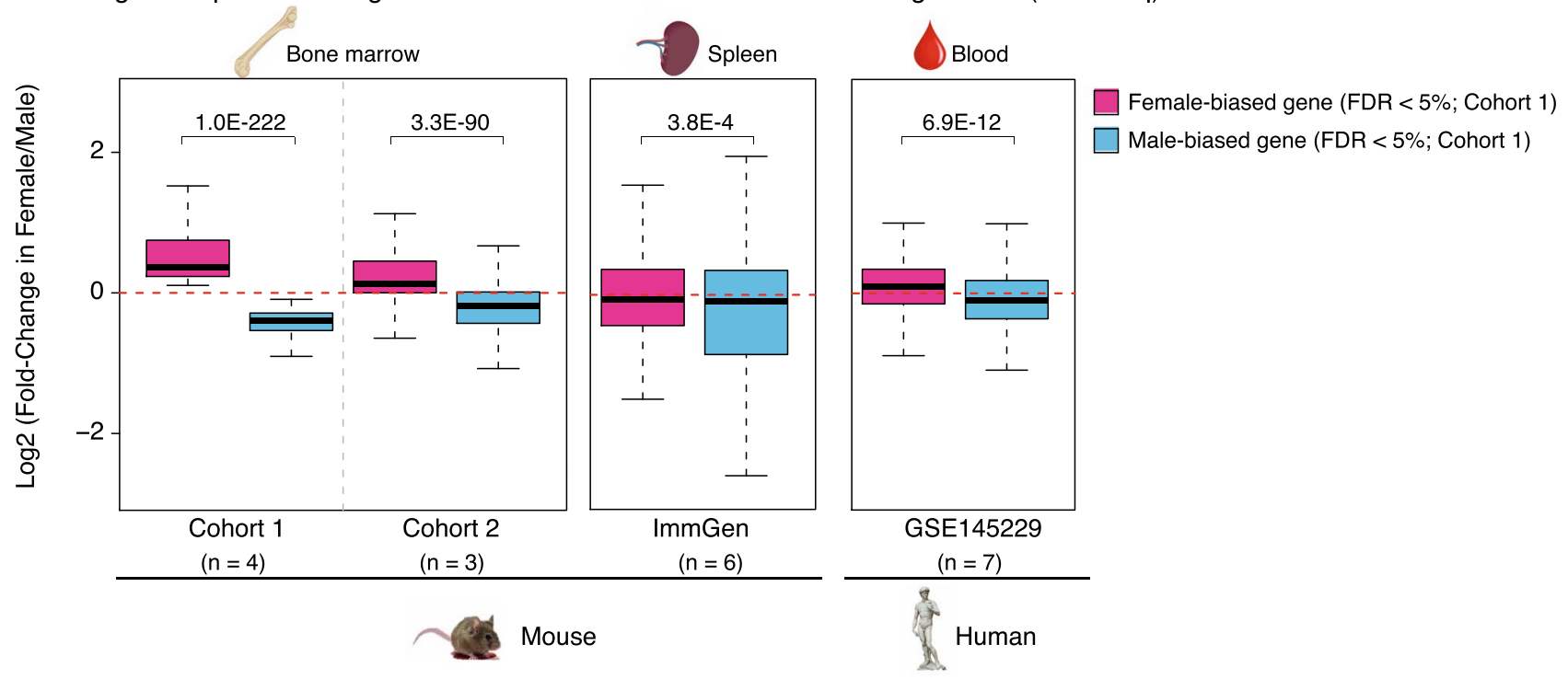

\section{b Fold-change in expression for genes with FDR $<5 \%$ in Cohort 1 with aging (RNA-seq)}

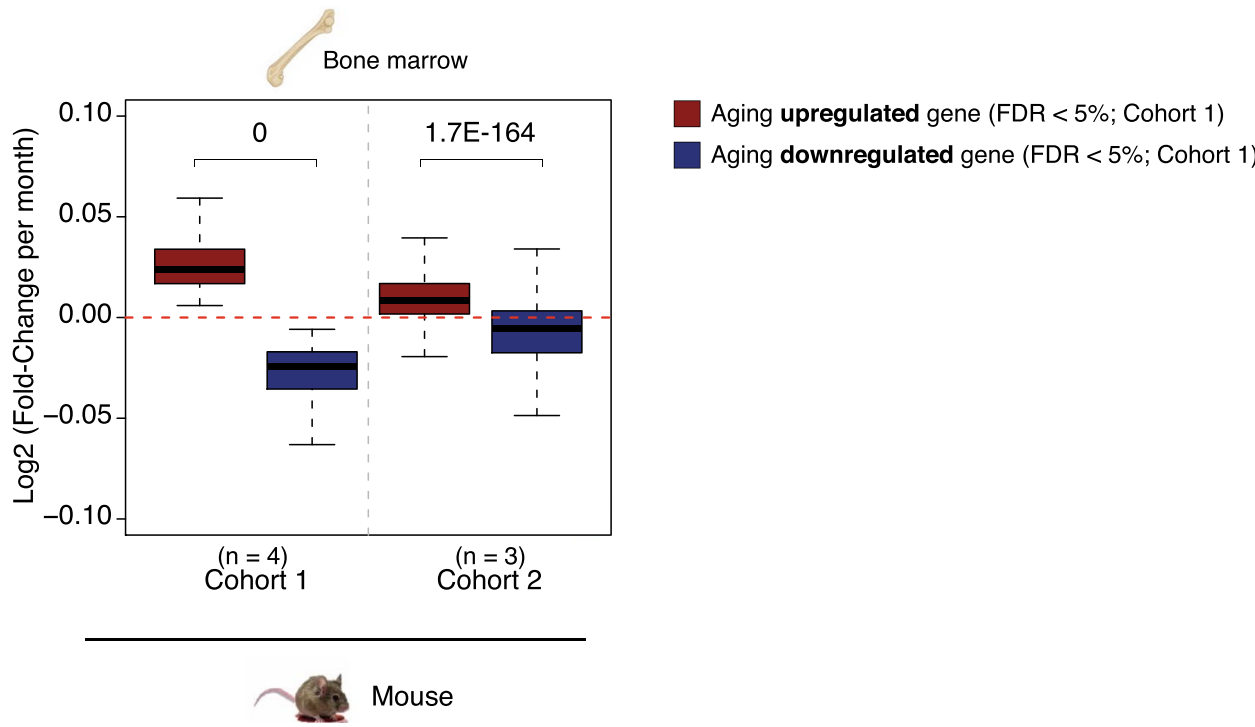

Extended Data Fig. 2 | Comparison to other neutrophil RNA-seq datasets. a, DESeq2 normalized $\log _{2}$ fold changes as a function of biological sex for differentially expressed mouse genes from our original cohort (FDR $<5 \%$ ) (and their human orthologs) across datasets [see Methods]. The original mouse bone marrow neutrophil cohort data are plotted for comparison as the leftmost panel. On the right are plotted corresponding log ${ }_{2}$ fold change values from our own smaller replication cohort of mouse bone marrow neutrophils ( $n=3$ per group), from mouse spleen neutrophils from ImmGen ${ }^{22}$ and a human blood neutrophil cohort ${ }^{24}$. P-values were calculated using a two-sided Wilcoxon test between female-biased and male-biased genes from our original cohort across new datasets, to test the robustness of such differences between our original cohort and new datasets. b. DESeq2 normalized $\log _{2}$ fold changes per month during aging for differentially age-regulated mouse genes from our original cohort (FDR $\left.<5 \%\right)$. The original mouse bone marrow neutrophil cohort data are plotted for comparison as the leftmost panel. On the right are plotted corresponding log fold change values from our own smaller replication cohort of mouse bone marrow neutrophils ( $n=3$ per group). P-values were calculated using a two-sided Wilcoxon test between upregulated vs. downregulated genes, to test the robustness of such differences between our original cohort $\log _{2}$ fold changes and a new dataset. The center line represents the sample median, the box limits consist of the 25th and 75th percentiles, the whiskers span $1.5 x$ the interquartile range, and each RNA-seq sample is represented by a point on the graph. 


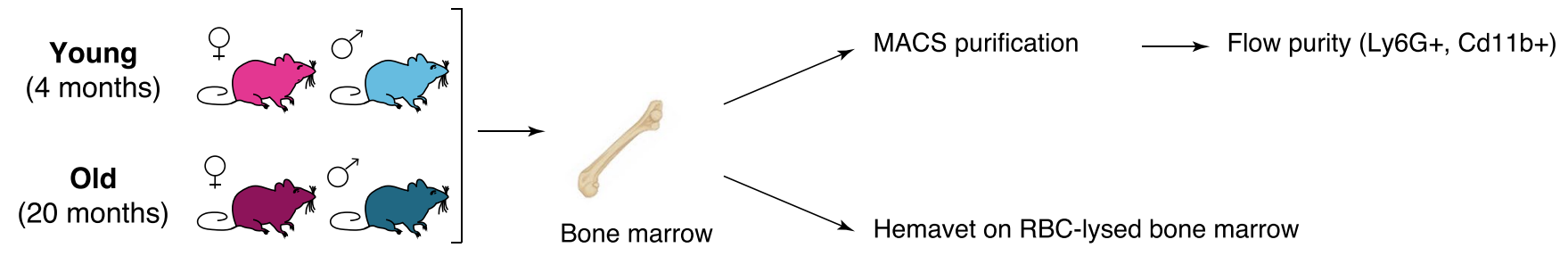

a Example gating for neutrophil purity flow (young female)

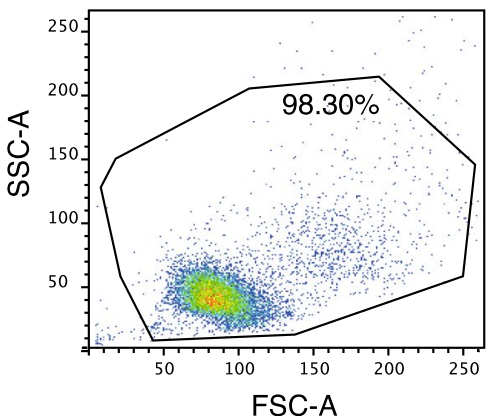

b Neutrophil purity by flow cytometry $(2$ cohorts)

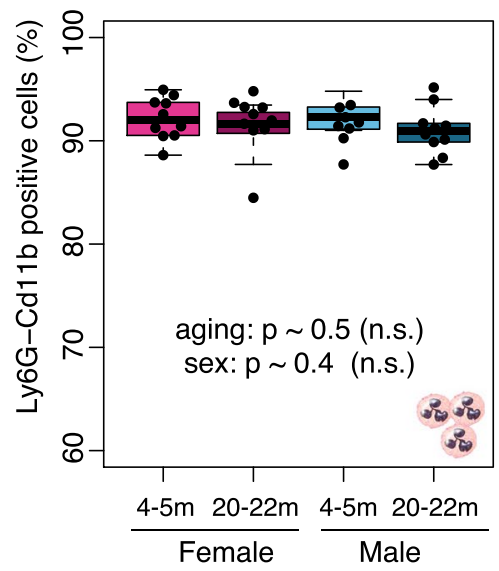

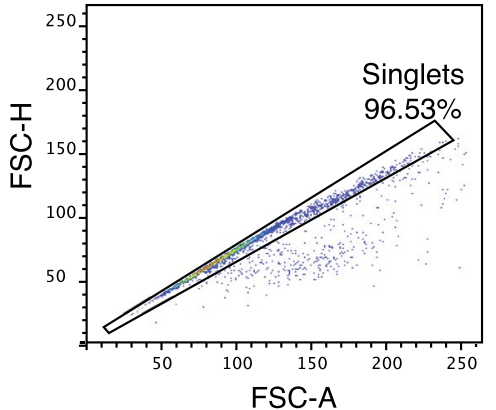

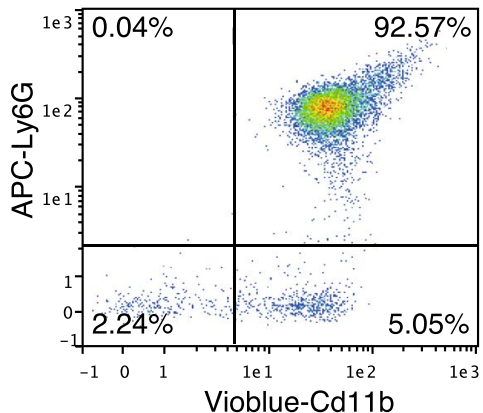

C Neutrophils proportion in bone marrow by HEMAVET (2 cohorts)

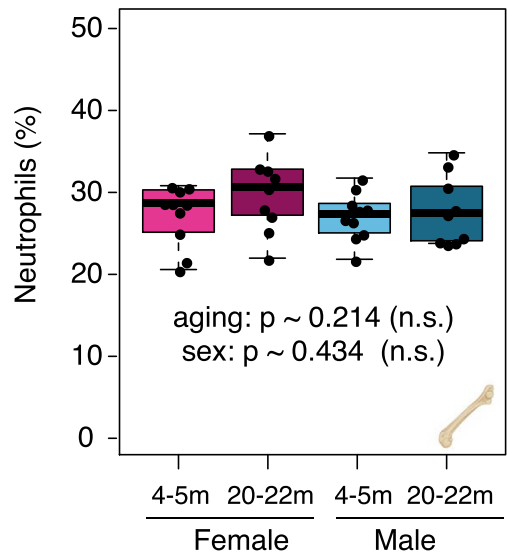

Extended Data Fig. 3 | Bone marrow neutrophil purity is not impacted by animal sex or organismal age. a, Representative flow cytometry gating strategy of bone-marrow neutrophils purified using MACS from a young female mouse. Neutrophils are expected to be double positive for Cd11b and Ly6G. b, Neutrophil purity from 2 independent cohorts of aging male vs. female mice ( $n=8$ for old females, and $n=10$ per group for all other groups), determined as the Cd11b + Ly6G + population. c, Proportion of neutrophils among bone marrow nucleated cells according to the Hemavet $950 \mathrm{FS}$ from 2 independent cohorts of aging male vs. female mice ( $n=9$ for old females, and $n=10$ per group for all other groups). Statistical analysis for (b) and (c) derived from a linear modeling analysis with sex and age as covariates, similar to our 'omic' analysis models, with significance of coefficients as reported by the R 'Im' function. The center line represents the sample median, the box limits consist of the 25 th and 75 th percentiles, the whiskers span $1.5 x$ the interquartile range, and each RNA-seq sample is represented by a point on the graph. 


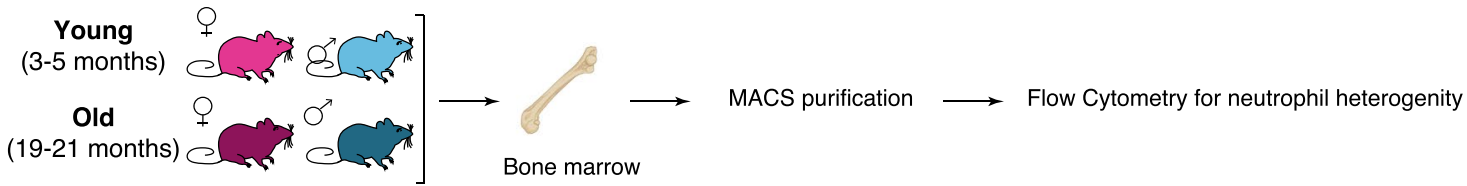

A Example gating for neutrophil heterogenity panel on MACS purified neutrophils (young female)
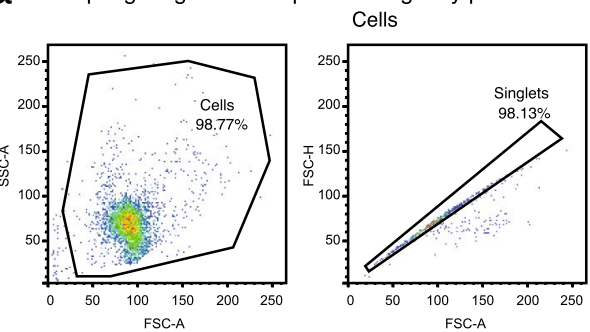

Singlets

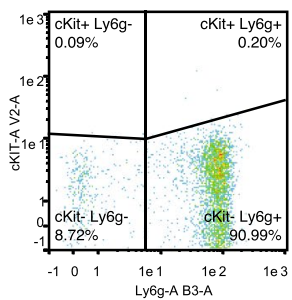

$\mathrm{Cd} 11 \mathrm{~b}+\mathrm{Ly} 6 \mathrm{~g}+$
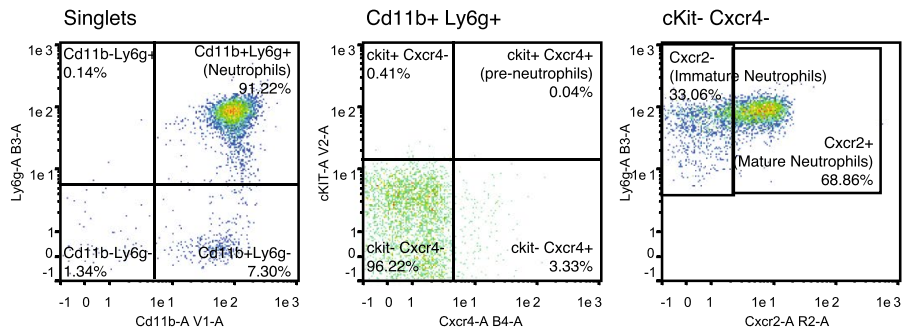
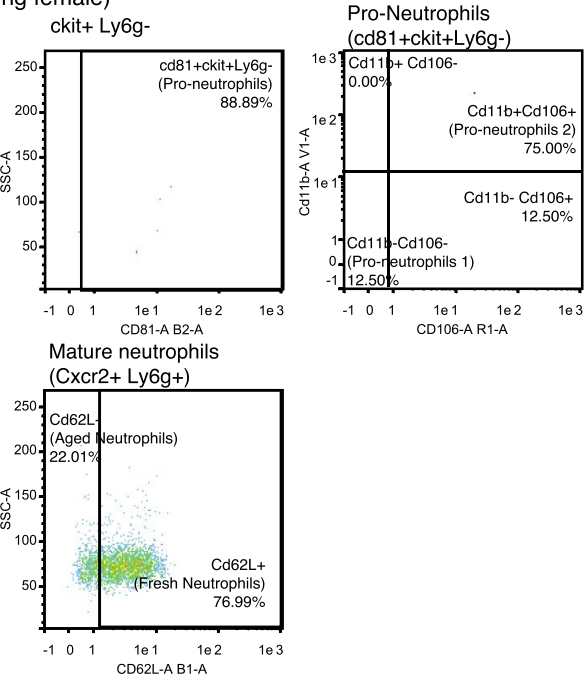

b Example gating for neutrophil heterogenity panel on RBC-lysed bone marrow cells (young female pool)

Cells

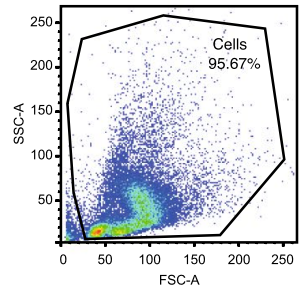

Singlets

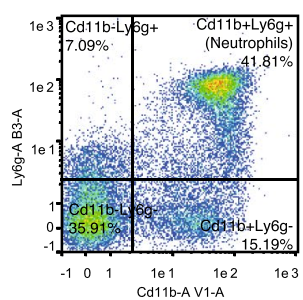

C Mature vs. Immature neutrophils (2 cohorts)

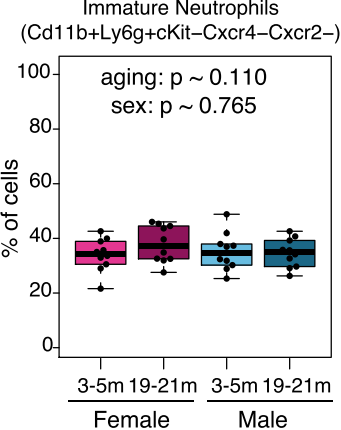

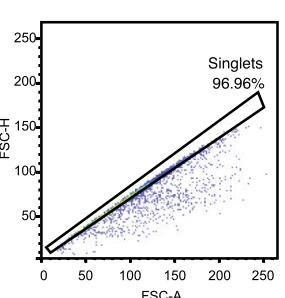

Cd11b+ Ly6g+

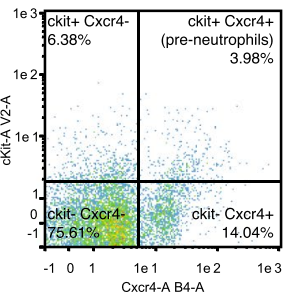

Mature Neutrophils

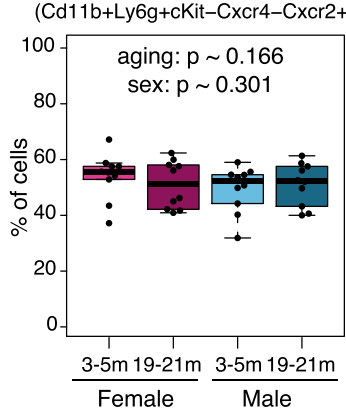

Singlets

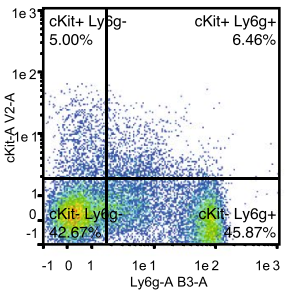

cKit- Cxcr4-

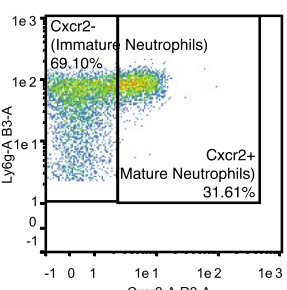

ckit+ Ly6g-

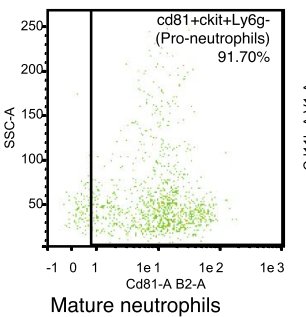

Mature neutrophils (Cxcr2+ Ly6g+)

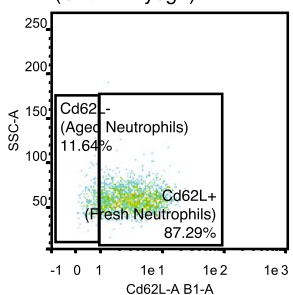

Pro-Neutrophils (cd81+ckit+Ly6g-)

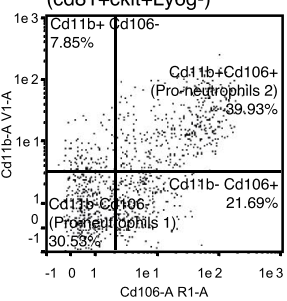

$\begin{array}{llllll}-1 & 0 & 1 & 1 \mathrm{e} 1 & 1 \mathrm{e} 2 & 1 \mathrm{e}\end{array}$

d Proportion of «fresh" vs «aged» mature neutrophils (2 cohorts)

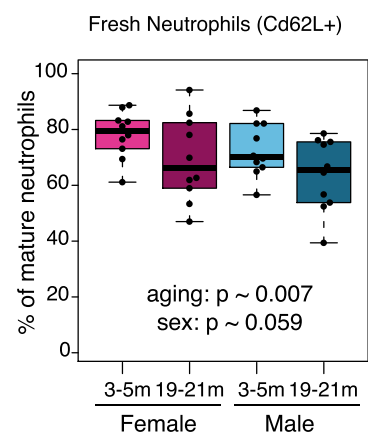

Aged Neutrophils (Cd62L-)

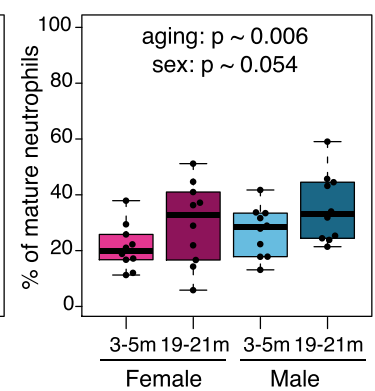

Extended Data Fig. 4 | See next page for caption. 
Extended Data Fig. 4 | Analysis of MACS-purified bone marrow neutrophil heterogeneity. a, Representative flow cytometry gating strategy of bonemarrow neutrophils purified using MACS from a young female mouse. Populations were defined according to previously published markers (see methods). Specifically, among live cell singlets, pro-neutrophils were defined as C-Kit+ ${ }^{+} \mathrm{Ly} 6 \mathrm{~g}-\mathrm{Cd} 81^{+}$, with subpopulation of pro-neutrophil 1 defined as c-Kit ${ }^{+}$Ly6G-Cd81+CD11b-CD106- and pro-neutrophils 2 as C-Kit ${ }^{+}$Ly6G-Cd81+CD11b+CD106 ${ }^{+}$. Pre-neutrophils were defined as Cd11 b ${ }^{+} \mathrm{Ly}^{-} 6 \mathrm{G}^{+} \mathrm{C}-\mathrm{Kit}^{+} \mathrm{C} \times \mathrm{Cr} 4^{+}$ cells. Immature neutrophils were defined as $\mathrm{CD} 11 \mathrm{~b}^{+} \mathrm{Ly} 6 \mathrm{G}^{+} \mathrm{C}-\mathrm{Kit}{ }^{-} \mathrm{CxCr} 4^{-} \mathrm{CxCr2}{ }^{-}$cells, and mature neutrophils as $\mathrm{CD} 11 \mathrm{~b}^{+} \mathrm{Ly} 6 \mathrm{G}^{+} \mathrm{C}-\mathrm{Kit}^{-} \mathrm{CxCr} 4^{-} \mathrm{CxCr} 2^{+}$. Fresh vs.

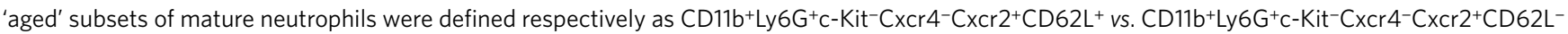
cells. The gate from which cells are plotted is indicated above each flow cytometry plot. $\mathbf{b}$, Representative flow cytometry gating strategy of RBC-lysed bone marrow cells from a young female mouse. The gate from which cells are plotted is indicated above each flow cytometry plot. This analysis was run as a control for the presence/abundance of $\mathrm{cKit}^{+}$cells, as MACS-purified cells had extremely low numbers of these cells. c, Amounts of immature (left) vs. mature (right) neutrophil among all MACS-purified cells from 2 independent cohorts of aging male vs. female mice ( $n=10$ per group). d, Proportion of 'fresh' vs. 'aged' neutrophils among mature neutrophils from 2 independent cohorts of aging male vs. female mice ( $n=10$ per group). Statistical analysis for (c) and (d) derived from a linear modeling analysis with sex and age as covariates, similar to our 'omic' analysis models, with significance of coefficients as reported by the R ' $I \mathrm{~m}$ ' function. The center line represents the sample median, the box limits consist of the 25th and 75th percentiles, the whiskers span $1.5 x$ the interquartile range, and each RNA-seq sample is represented by a point on the graph. Also see Supplementary Table 2 for quantifications of all populations. 
a Top GSEA enrichments for age-regulation from RNA-seq (Gene Ontology)

GO:0032456_endocytic recycling G0:0005788_endoplasmic reticulum lumen GO:1901800_positive regulation of proteasomal protein catabolic process GO:0090527_actin filament reorganization GO:0043522 leucine zipper domain binding GO:0006544_glycine metabolic process GO:0034976_response to endoplasmic reticulum stress GO:0050897 cobalt ion binding GO:0042605_peptide antigen binding GO:2000249_regulation of actin cytoskeleton reorganization GO:0000776_kinetochore GO:0005643_nuclear pore GO:0007094 mitotic spindle assembly checkpoint GO:0000070_mitotic sister chromatid segregation GO:0017056_structural constituent of nuclear pore GO.0000777 condensed chromosome kinetochore GO:0000724_double-strand break repair via homologous recombination GO:0000775_chromosome, centromeric region GO:0007059_chromosome segregation GO:0005694_chromosome

b Top GSEA enrichments for age-regulation from RNA-seq (KEGG)

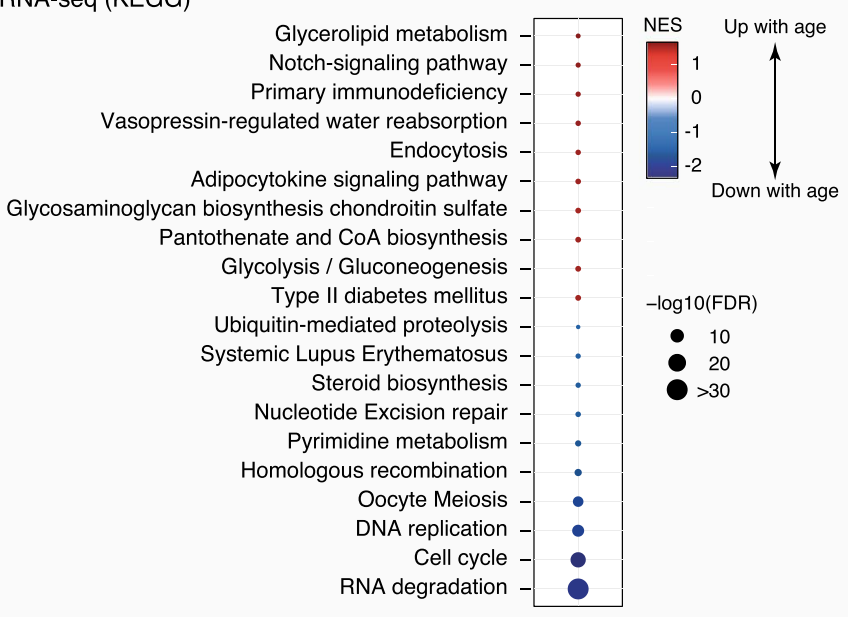

C Padi4 gene expression(RNA-seq) d Representative Western Blots

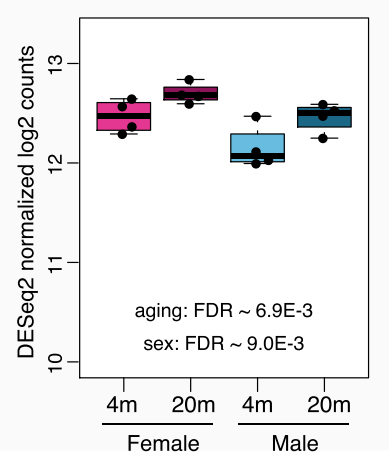

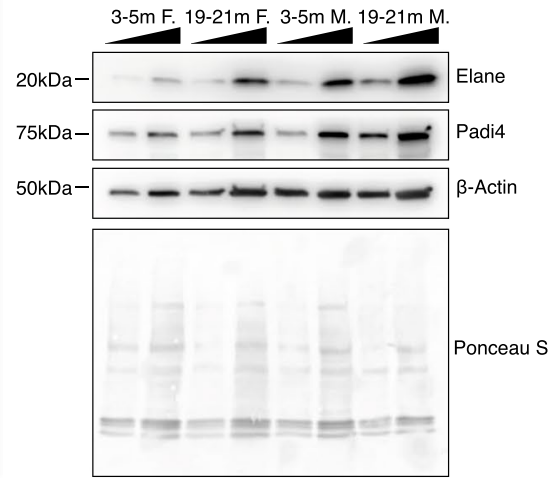

e Western Blot protein expression quantification

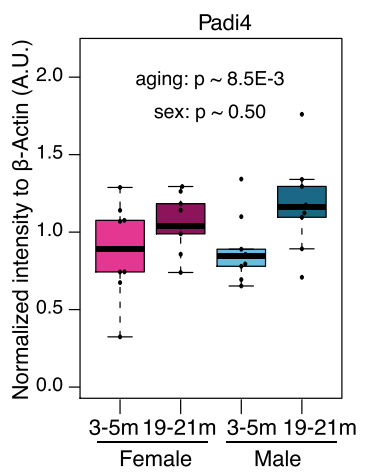

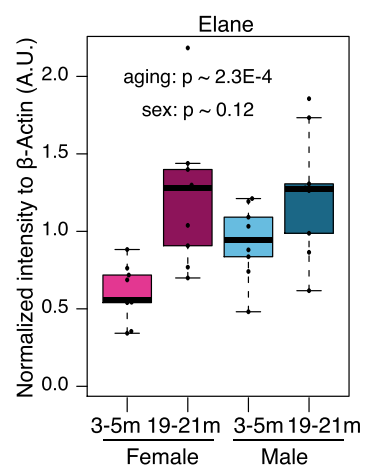

Extended Data Fig. 5 | See next page for caption. 
Extended Data Fig. 5 | Regulated pathways in bone marrow neutrophils during aging reveals downregulation of chromatin-related pathways (continued). a-b, Top enriched gene sets from Gene Ontology (a) and KEGG (b) using GSEA for differential RNA expression. Only the top 10 most upand top 10 most downregulated gene sets are plotted for readability. Full lists and statistics available in Supplementary Table 2. Shown pathways with FDR $<5 \%$. c, Boxplot of Padi4 transcriptional levels from RNA-seq. Significance: DESeq2 FDR values for regulation as a function of aging or biological sex. The center line represents the sample median, the box limits consist of the 25 th and 75 th percentiles, the whiskers span $1.5 x$ the interquartile range, and each RNA-seq sample is represented by a point on the graph ( $n=4$ per group). d, Representative Western Blot images for primary bone marrow neutrophils for Elane, Padi4 and $\beta$-actin (loading control), as well as Ponceau S staining of PVDF blotting membrane. The membrane was cut along predicted molecular weights, and each strip was probed for each specific protein in that range. e, Boxplot of quantification of Western Blot for Padi4 (left) and Elane (right) from primary bone marrow neutrophils. Data from 2 independent cohorts of aging male vs. female mice ( $n=9$ per group). Two concentrations of the same sample were loaded side by side for each biological sample. Statistical analysis derived from a linear modeling analysis with sex and age as covariates, similar to our 'omic' analysis models, with significance of coefficients as reported by the R 'Im' function. The center line represents the sample median, the box limits consist of the 25th and 75th percentiles, the whiskers span 1.5x the interquartile range, and each RNA-seq sample is represented by a point on the graph. All uncropped western blot images and quantification data is been made available on Figshare (https://doi. org/10.6084/m9.figshare.14154665.v1). NES: Normalized Enrichment Score (for GSEA analysis). FDR: False Discovery Rate. 
a Example neutrophil propidium lodide cell cycle analysis (young male)
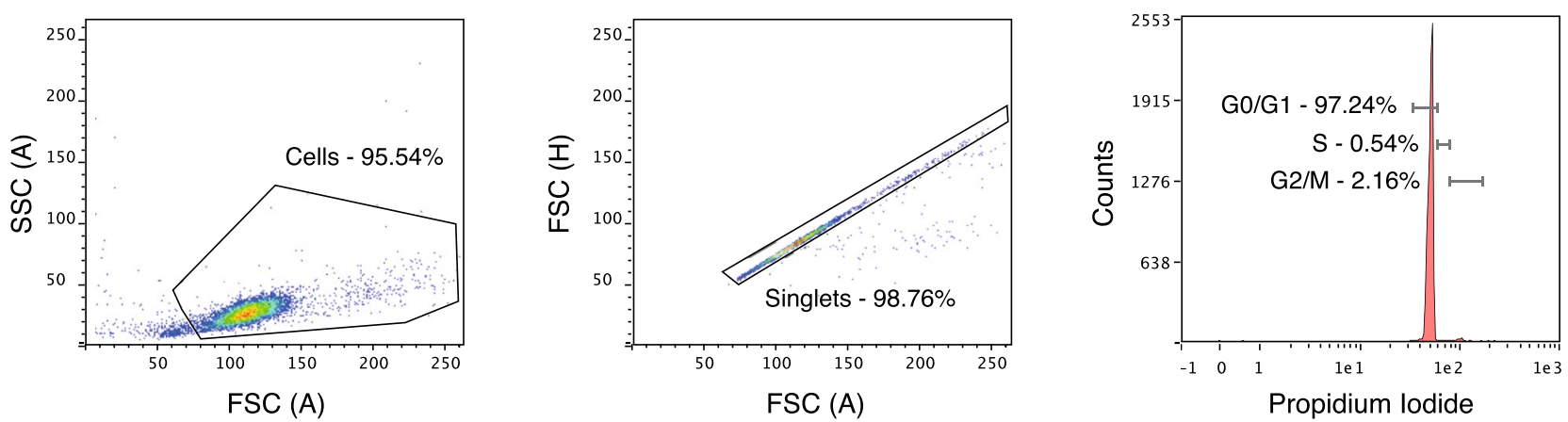

b Observed cell cycle phases in MACS-purified neutrophils
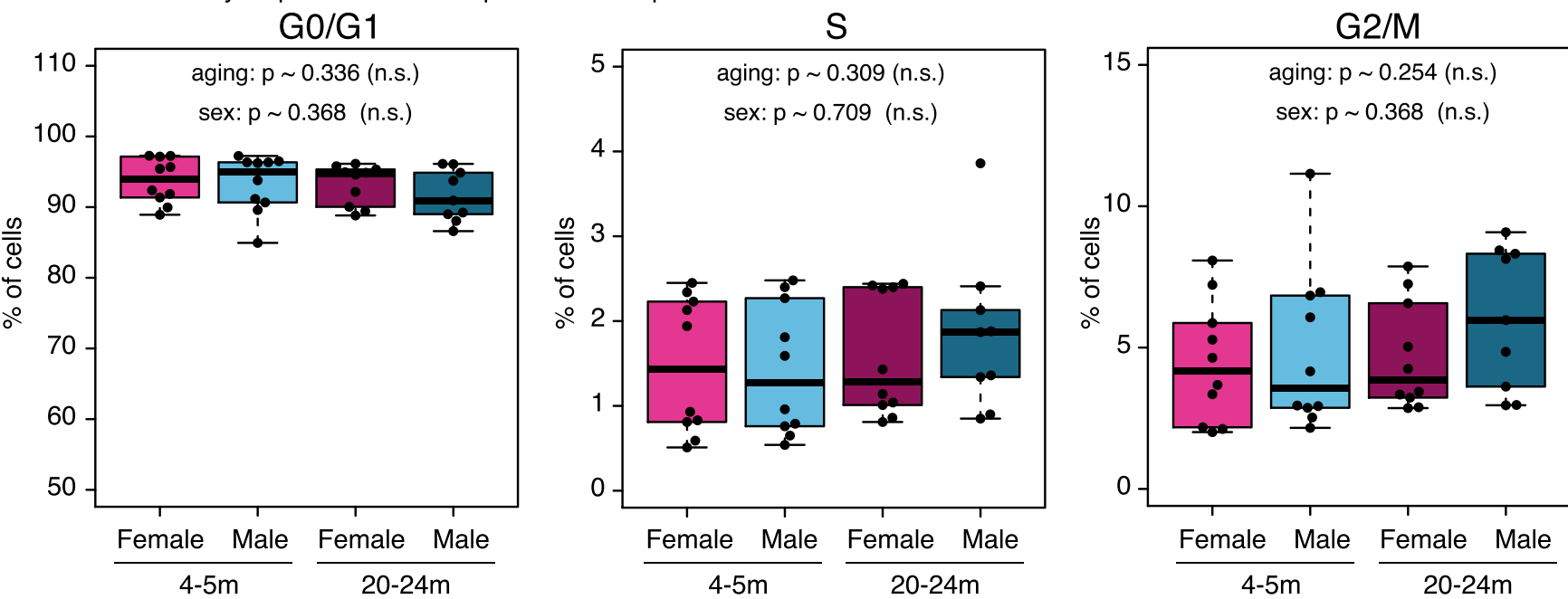

Extended Data Fig. 6 | The distribution of cell cycle phases of MACS-purified bone marrow neutrophil is not impacted by animal sex or organismal age. a, Representative flow cytometry gating strategy of bone-marrow neutrophils purified using MACS from a young male mouse stained using Propidium lodide to assess cell-cycle phase based on DNA content. Neutrophils are expected to be largely post-mitotic. $\mathbf{b}$, Boxplots of MACS-purified bone marrow neutrophil cell cycle distribution (GO/G1, S or G2/M) from 2 independent cohorts of aging male vs. female mice $(n=9$ for old males, and $n=10$ per group for all other groups), determined by DNA content. The overwhelming majority of samples have $>90 \%$ cells in G0/G1, and there are no trends associated to organismal age or biological sex. Statistical analysis for (b) derived from a linear modeling analysis with sex and age as covariates, similar to our 'omic' analysis models, with significance of coefficients as reported by the R 'Im' function. The center line represents the sample median, the box limits consist of the 25 th and 75 th percentiles, the whiskers span $1.5 x$ the interquartile range, and each RNA-seq sample is represented by a point on the graph. 
a Top GSEA enrichments for sex-regulation from RNA-seq (Gene Ontology)

GO:0002376_immune system process GO:0002476_antigen processing and presentation of endogenous peptide antigen via MHC class Ib GO:0050853_B cell receptor signaling pathway GO:0035556_intracellular signal transduction GO:0007411_axon guidance GO:0046977_TAP binding GO:0016477_cell migration

GO:0002250 adaptive immune response GO:0009897_external side of plasma membrane GO:0006955_immune response GO:0000775 chromosome, centromeric region GO:0000776_kinetochore GO:0005694_chromosome GO:0000777 condensed chromosome kinetochore GO:0007059_chromosome segregation GO:0000922 spindle pole GO:0007049_cell cycle GO:0006281_DNA repair

GO:0000070 mitotic sister chromatid segregation GO:0051301_cell division

b Top GSEA enrichments for sex-regulation from RNA-seq (KEGG)

Intestinal immune network for IgA production T-cell receptor signaling pathway Leukocyte transendothelial migration

Primary immunodeficiency GnRH signaling pathway MAPK signaling pathway VEGF signaling pathway Cell adhesion molecules (CAM) Cytokine-cytokine receptor interaction Focal adhesion Systemic lupus erythematosus DNA replication Cell cycle Mismatch repair Ribosome

Nucleotide excision repair Aminoacyl tRNA biosynthesis Homologous recombination Spliceosome

Parkinson's disease

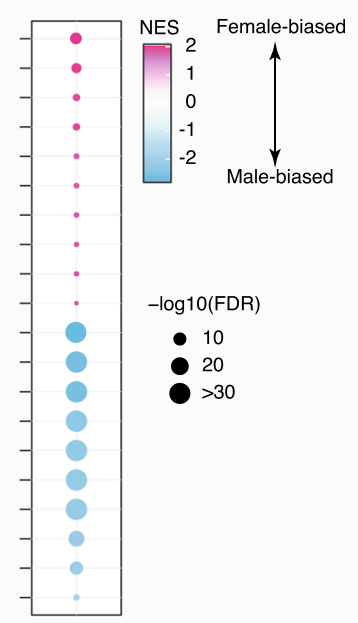

d Boxplot of lipid droplet-related gene expression in neutrophils (RNA-seq)

C MetaboAnalyst PSEA scatter plot (KEGG)
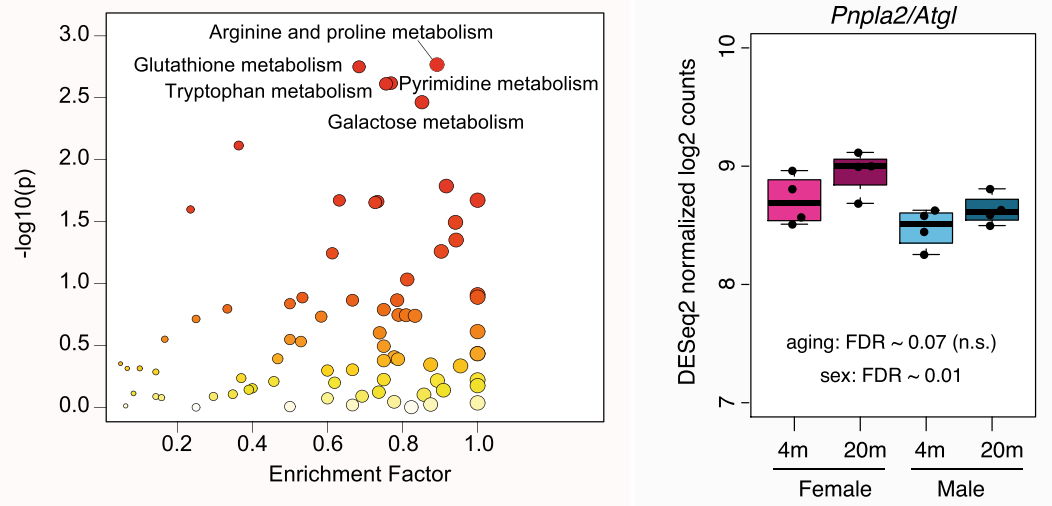
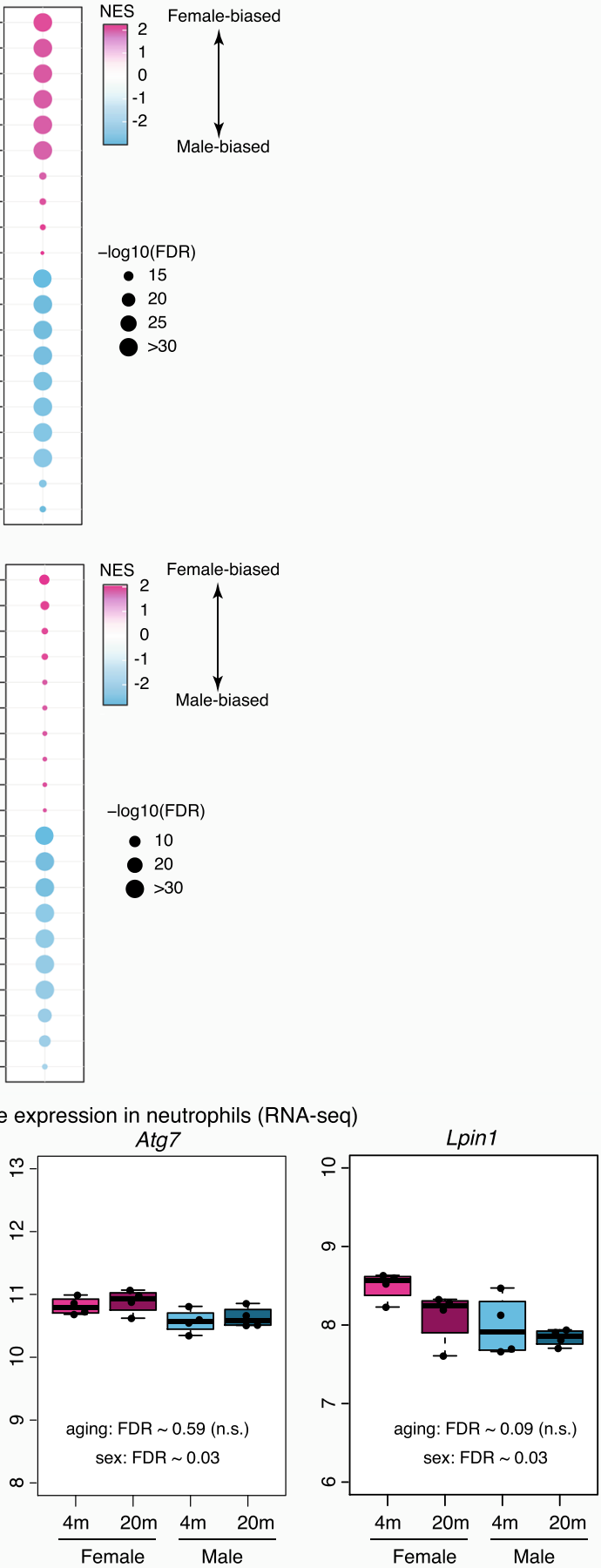

e
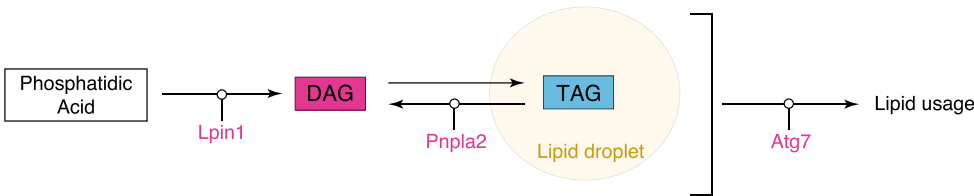

\section{Extended Data Fig. 7 | See next page for caption.}


Extended Data Fig. 7 | Sex-dimorphic pathways in bone marrow neutrophils reveal differential regulation of chromatin-related pathways (continued). a-b, Top enriched gene sets from Gene Ontology (a) and KEGG (b) using GSEA for differential RNA expression as a function of sex. Only the top 10 most up- and top 10 most downregulated gene sets are plotted for readability. Full lists and statistics available in Supplementary Table 2. All shown pathways and genes such as FDR <5\%. c, MetaboAnalyst PSEA scatterplot for KEGG pathways from metabolomics. NES: Normalized Enrichment Score (for GSEA analysis). FDR: False Discovery Rate. d, Boxplot of transcriptional levels from our RNA-seq for Pnpla2/Atgl, Atg7 and Lpin1, which are sex-dimorphic lipid metabolism-related genes. The significance is derived from DESeq2, and we report the corresponding FDR values for regulation as a function of aging or biological sex. The center line represents the sample median, the box limits consist of the 25th and 75th percentiles, the whiskers span $1.5 x$ the interquartile range, and each RNA-seq sample is represented by a point on the graph ( $n=4$ per group). $\mathbf{e}$, Summary scheme of discussed lipid usage in female vs. male neutrophils based on lipidomic and transcriptomic data. Catalyzed reactions are derived from Wikipathway WP3901 (Lipid droplet metabolism) and ${ }^{35}$. 
a Nucleosome "shadow" (NucleoATAC v-plots)
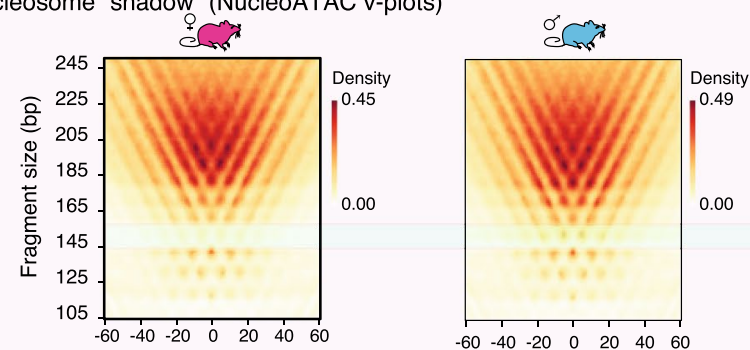

b Xist-associated peaks ATAC-seq signal

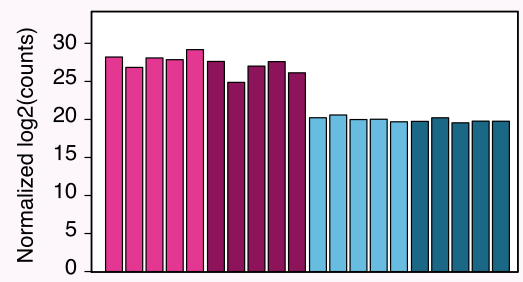

C Y chromosome peaks ATAC-seq signal

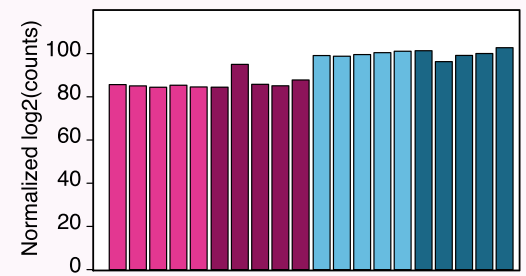

d ATAC-seq (peak accessibility)
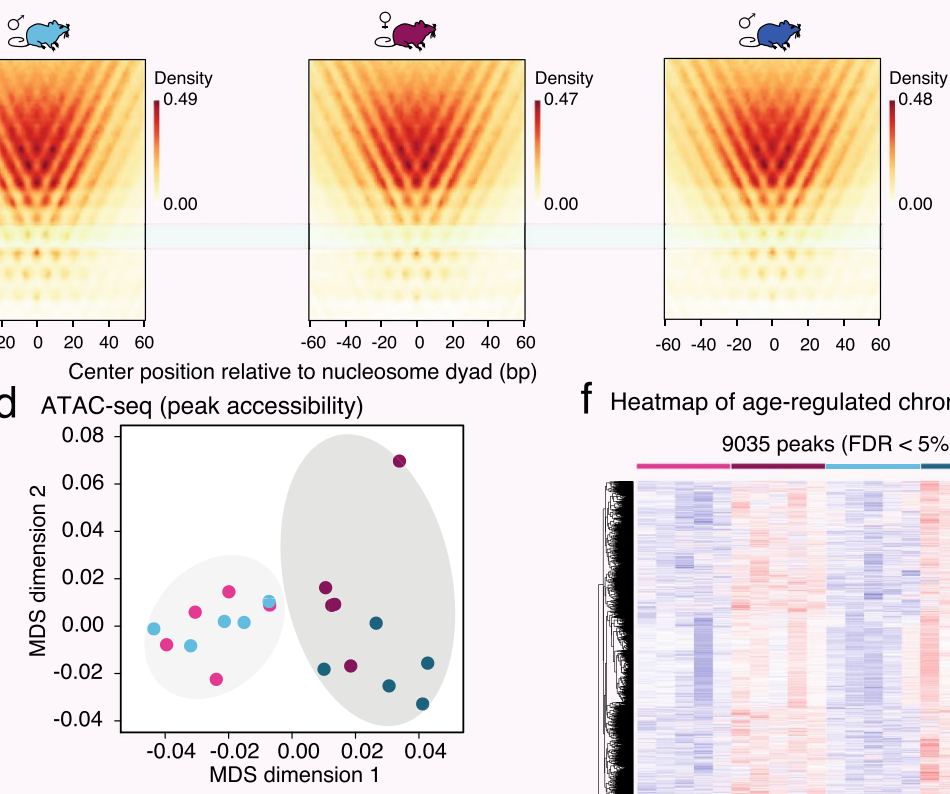

D Differential accessibility statistics by ATAC-seq

\begin{tabular}{lcc}
\hline & All chromosomes & Autosomes \\
\hline Aging regulation & & \\
- Increased accessibility & 5108 & \\
- Decreased accessibility & 3927 & \\
Sex-dimorphic regulation & & \\
- Female-biased & 478 & 346 \\
- Male-biased & 260 & 251 \\
\hline
\end{tabular}

g Heatmap of sex-dimorphic chromatin accessibility i Nucleosome occupancy (All nucleosome calls)

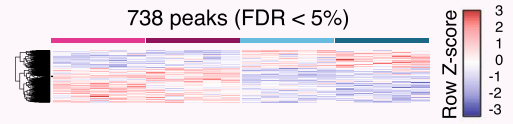

TNNMN

h Peaks with sex-dimorphic accessibility $(\mathrm{FDR}<5 \%)$

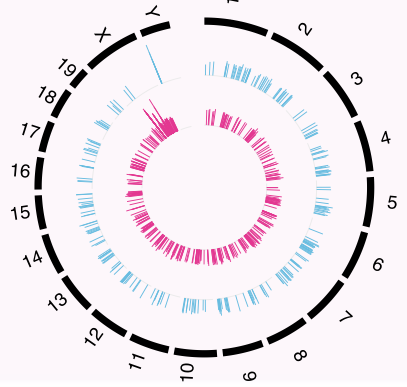

K Heatmap of age-regulated repetitive elements (RNA-seq)
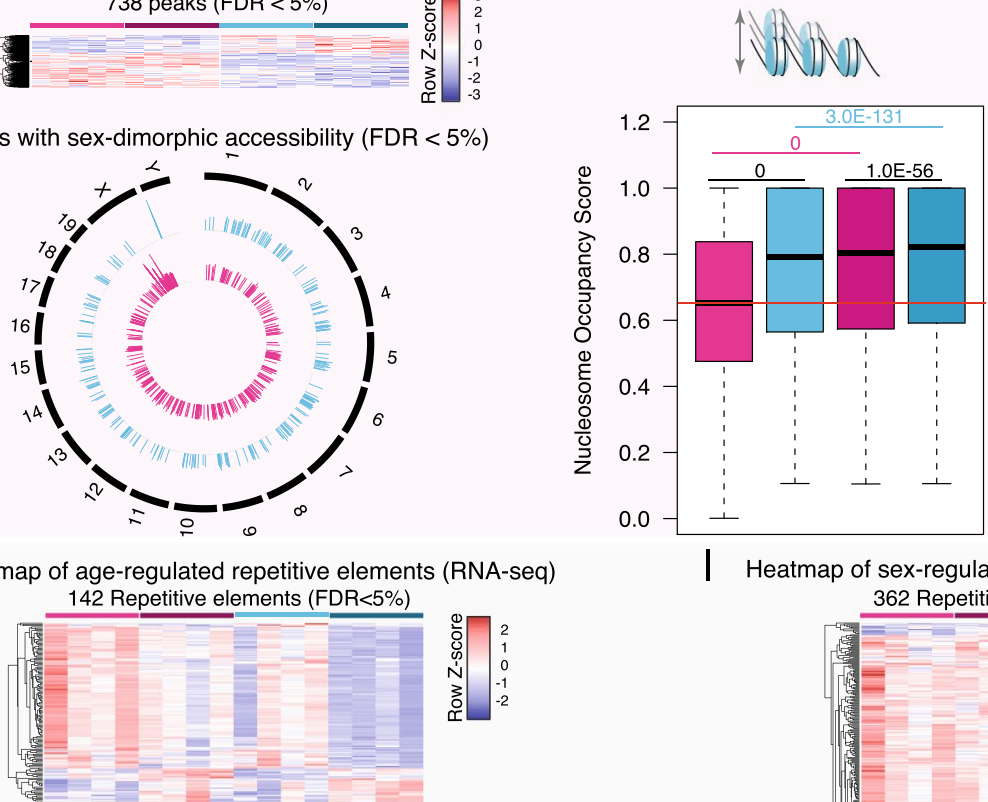

f Heatmap of age-regulated chromatin accessibility

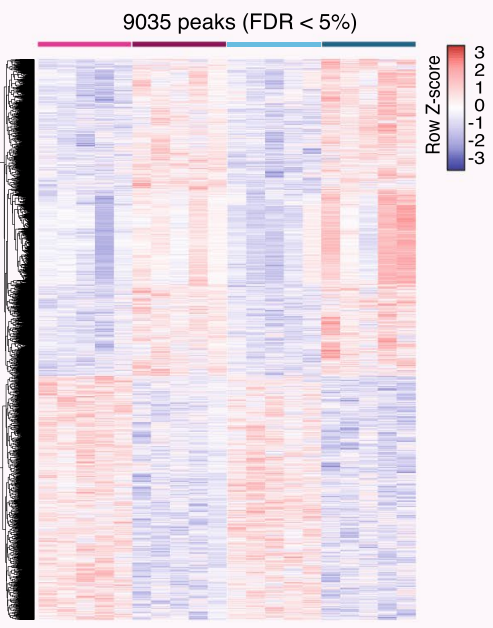

j ATAC fragment size at Peaks
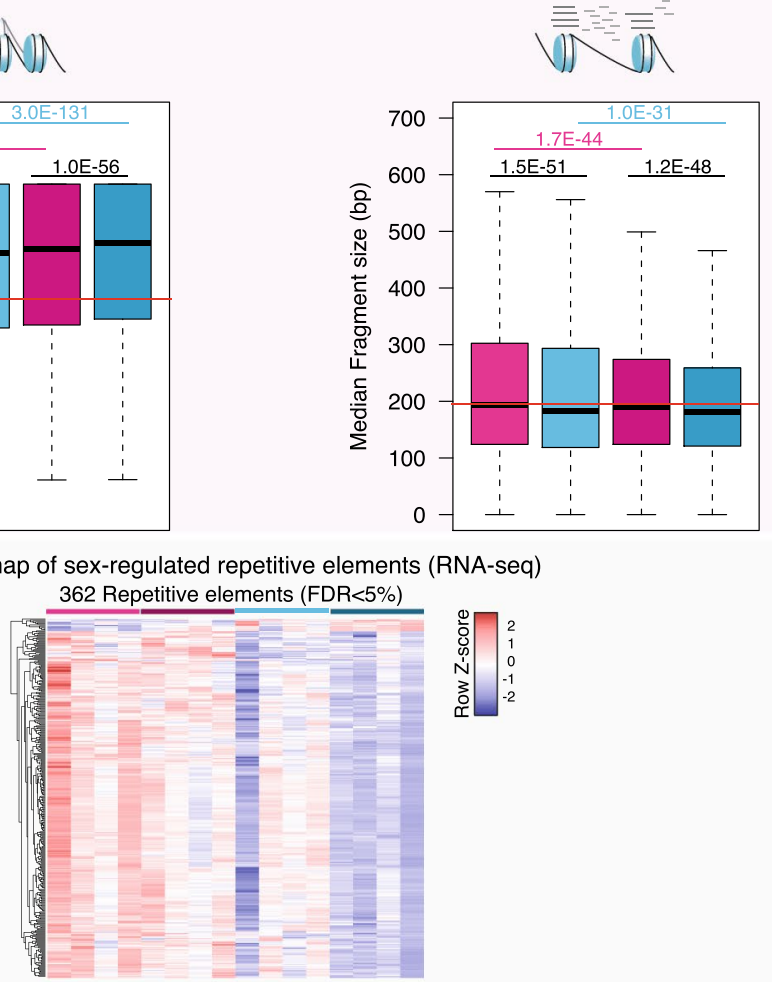

Young females (4 months)

Old females (20 months)

Young males (4 months)

Old males (20 months)

Extended Data Fig. 8 | See next page for caption. 
Extended Data Fig. 8 | ATAC-seq analysis reveals age- and sex-related differences in the chromatin architecture of bone marrow neutrophils

(continued). a, NucleoATAC v-plots. The light green box overlay reveals a v-plot region where males have higher signal than females, regardless of age, suggesting differences in nucleosomal architecture. $\mathbf{b}$-c, Barplot of DESeq2-normalized log2 counts at ATAC-seq peaks associated to Xist (b) or situated on the $Y$ chromosome (c), showing the expected pattern between male and female samples. $\mathbf{d}$, Multidimensional Scaling analysis results of chromatin accessibility from ATAC-seq. e, Table of significantly differentially accessible ATAC-seq peaks as a function of organismal age and sex. The number of significant peaks located on autosomes (that is not on chromosomes $\mathrm{X}$ or $\mathrm{Y}$ ) is also reported. $\mathbf{f}-\mathrm{g}$, Heatmap of accessibility at peaks with significant ageregulated (f) or sex-dimorphic $(\mathbf{g})$ change in accessibility (FDR $<5 \%$ ). $\mathbf{h}$. Circular genome plot of the genomic positions of peaks with significant sex-bias in neutrophil ATAC-seq (FDR $<5 \%)$. i, Boxplot of nucleosome occupancy as calculated by NucleoATAC for all called nucleosomes across groups. Also see Fig. 4d. j, Boxplot of median ATAC-seq fragment length at ATAC-seq peaks across experimental groups. The horizontal red line in panels (i,j) shows the median value in neutrophils from young females for ease of comparison. Significance in non-parametric two-sided Wilcoxon rank-sum tests are reported for panels (i,j). Black p-values represent differences between male vs. female neutrophils in each age group; pink (blue) p-values represent age-related differences in female (male) neutrophils. For boxplots in panels $(\mathbf{i}, \mathbf{j})$, the center line represents the sample median, the box limits consist of the 25th and 75 th percentiles, the whiskers span $1.5 x$ the interquartile range, for readability and consistent with practices in genomics, outliers are not shown on the graph although they are always taken into account for the statistics. $\mathbf{k}-\mathbf{I}$, Heatmap of repetitive elements with significant age-regulated (k) or sexdimorphic (I) change in transcription from RNA-seq (FDR $<5 \%$ ). 

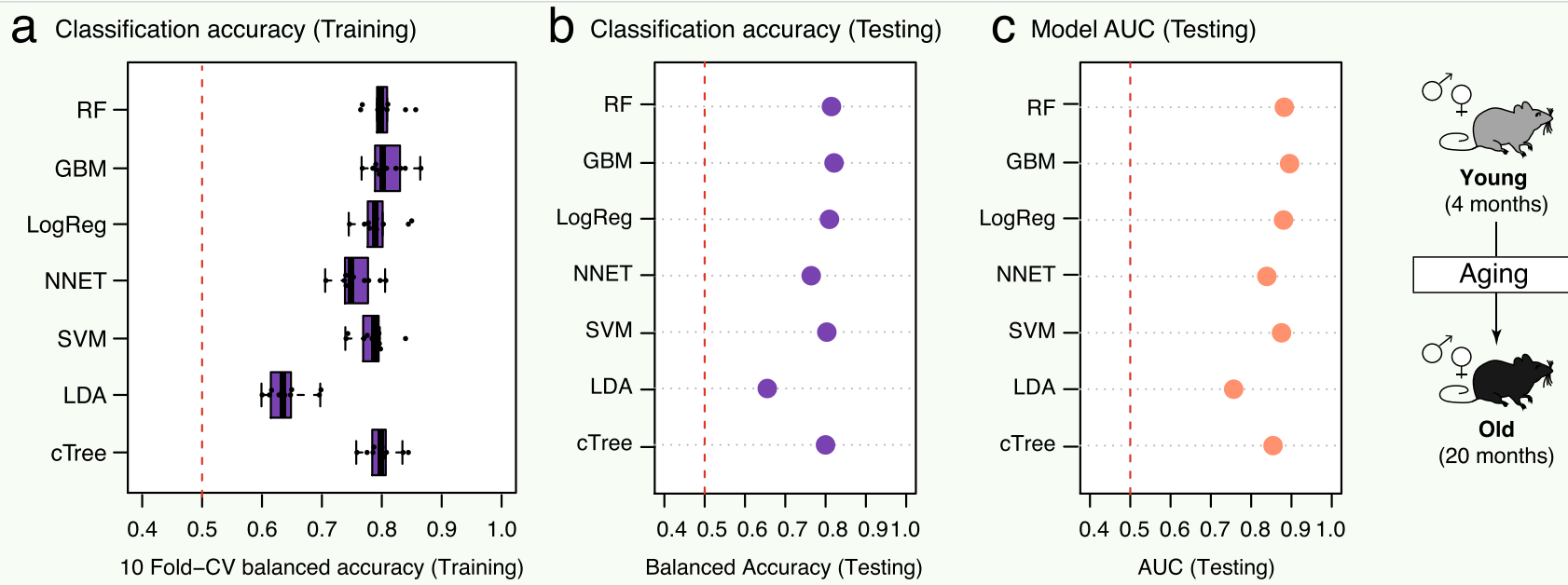

d Classification accuracy (Training)

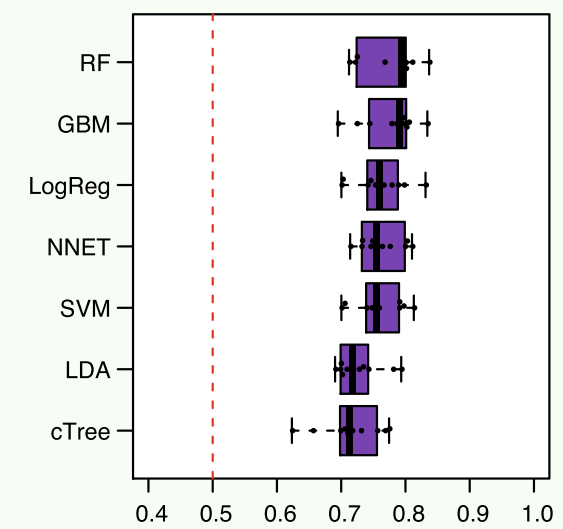

e Classification accuracy (Testing)

\section{f Model AUC (Testing)}
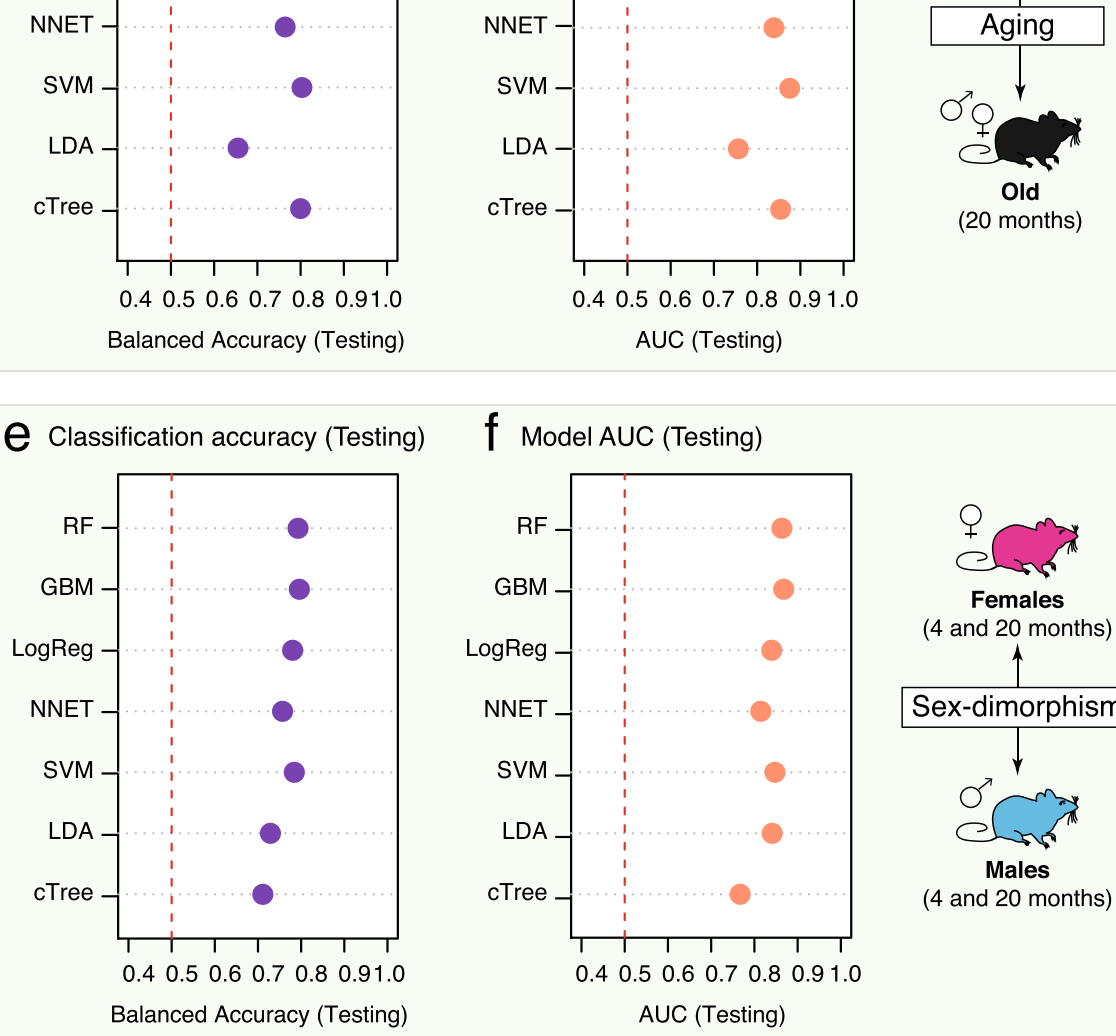

(20 months)

10 Fold-CV balanced accuracy (Training)

9 Top TF predictors of age-related expression by RNA-seq

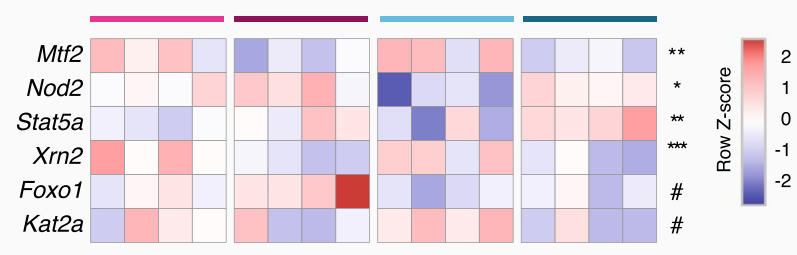

Age regulation

Young females (4 months) h Top TF predictors of sex-dimorphic expression by RNA-seq

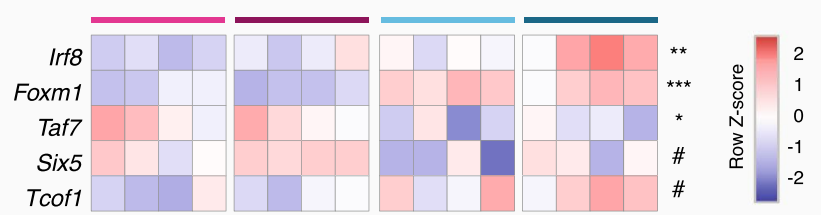

Sex regulation

Old females (20 months)

Young males (4 months)

Old males (20 months)

Extended Data Fig. 9 | Machine-learning analysis reveals that age-regulated and sex-dimorphic gene expression can be predicted by genomic and epigenomic features. a-c, Age-related machine-learning model performance metrics: balanced classification accuracy over the 10 cross-validation folds during model training (a), balanced classification accuracy on held-out testing data (b), and Area Under the Curve [AUC] on held-out testing data (c). Also see Supplementary Table 5A. d-f, Sex-dimorphism machine-learning model performance metrics: balanced classification accuracy over the 10 crossvalidation folds during model training (d), balanced classification accuracy on held-out testing data (e), and Area Under the Curve [AUC] on held-out testing data (f). Also see Supplementary Table 5C. g-h, Heatmap of RNA-seq expression level for top TF predictors in age-regulated (g) or sex-dimorphic (h) gene expression models with FDR $<10 \%$. DESeq2 FDR is reported on each line, with \#: FDR $<0.1$; ${ }^{*}$ FDR $<0.05$; ${ }^{\star \star}:$ FDR $<0.01$; ${ }^{\star \star \star}$ : FDR $<0.001$. FDR: False Discovery Rate. For boxplots in panels $(\mathbf{a}, \mathbf{d})$, the center line represents the sample median, the box limits consist of the $25^{\text {th }}$ and $75^{\text {th }}$ percentiles, the whiskers span $1.5 x$ the interquartile range. 


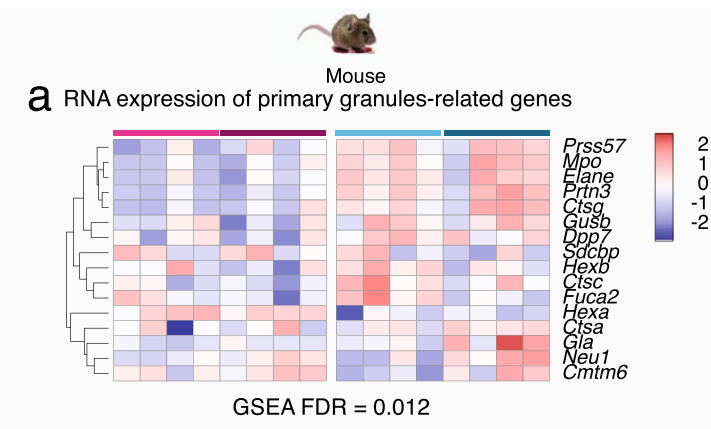

b RNA expression of secondary granules-related genes

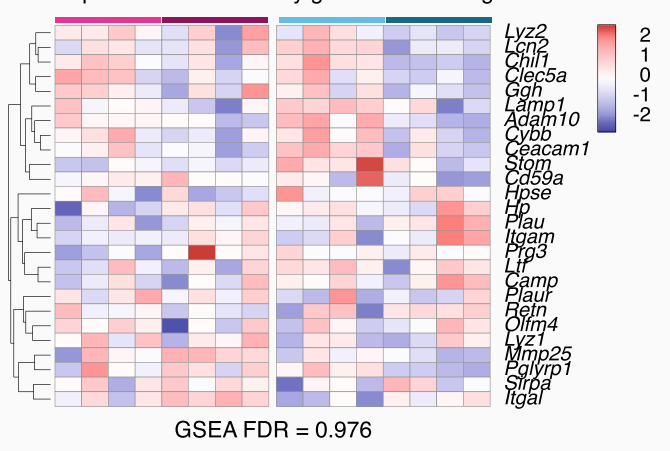

C RNA expression of tertiary granules-related genes

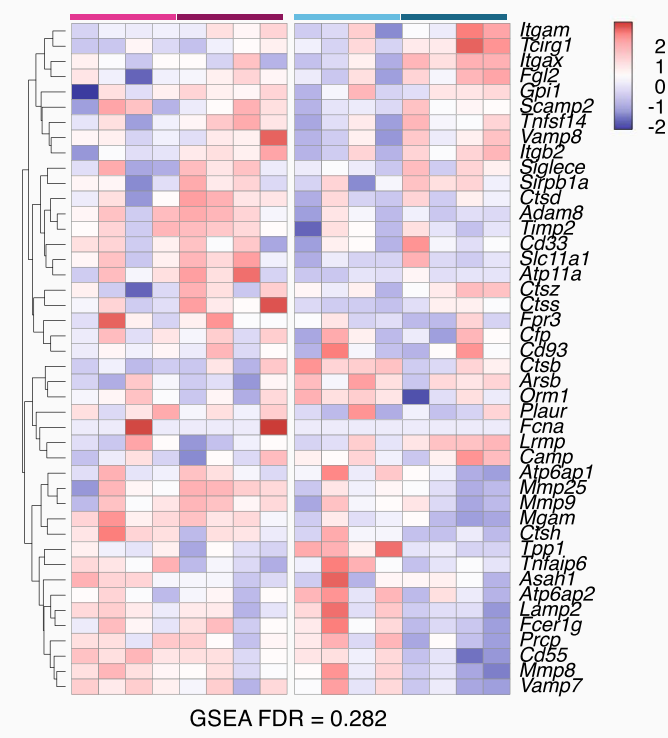

d RNA expression of sex-linked genes (Sex-bias control set)

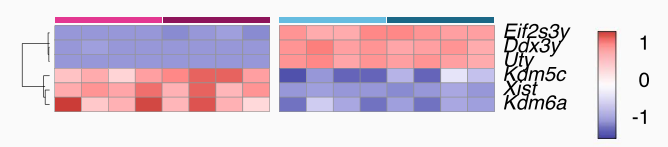

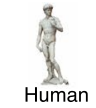

e Correlation plot of DDX3Y vs. ELANE protein expression

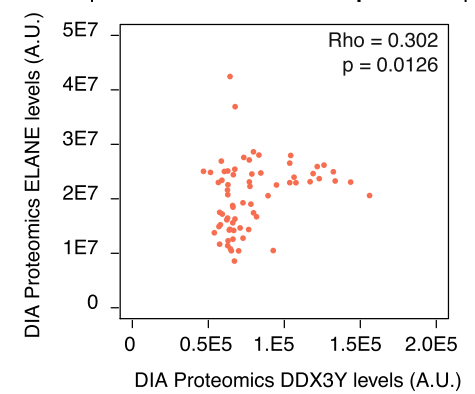

f Correlation plot of DDX3Y vs. MPO protein expression

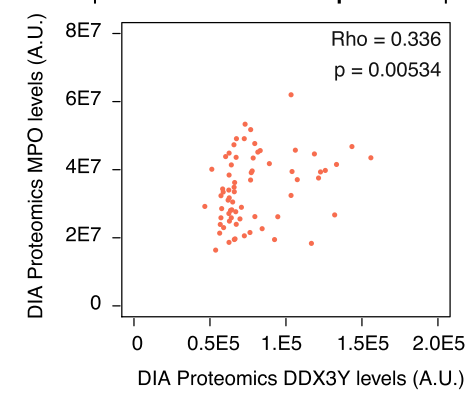

9 Correlation plot of DDX3Y vs. PRTN3 protein expression

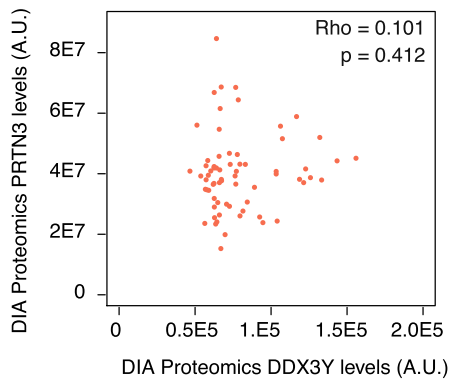

h Correlation plot of DDX3Y vs. CTSG protein expression

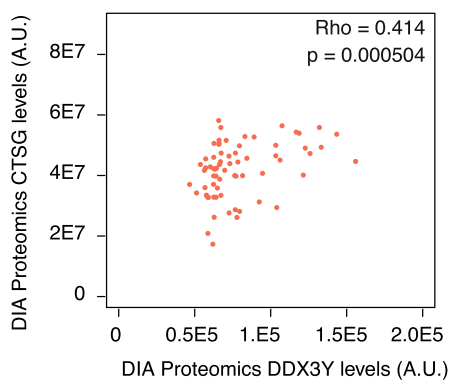

Extended Data Fig. 10 | Male neutrophils express higher levels of primary granule genes but not secondary and tertiary granule genes. a-c, Heatmap of normalized gene expression for primary (a), secondary (b) and tertiary (c) granule-related gene expression in our RNA-seq dataset. The estimated False Discovery Rate [FDR] using GSEA for each gene set is reported. d, Heatmap of normalized gene expression for X-linked (Kdm5c, Kdm6a, Xist) and Y-linked (Uty, Ddx3y, Eif2s3y) genes in our RNA-seq dataset. Panel (a) is identical to Fig. 7a to help direct comparison between sex-bias expression patterns of different granule types as well as canonical sex-biased genes. e-h, Scatterplots of protein levels of DDX3Y (as a proxy for likely of a sample being derived from a male donor), compared to protein levels for primary granule-related proteins ELANE (e), MPO (f), PRTN3 (g) and CTSG (h) in neutrophils from healthy human donors. Normalized data was obtained from the supplementary material of ${ }^{97}$. Estimates of Spearman Rank correlation (Rho) and significance of correlation are reported for each correlation on the scatterplot. 


\section{Reporting Summary}

Nature Research wishes to improve the reproducibility of the work that we publish. This form provides structure for consistency and transparency in reporting. For further information on Nature Research policies, see our Editorial Policies and the Editorial Policy Checklist.

\section{Statistics}

For all statistical analyses, confirm that the following items are present in the figure legend, table legend, main text, or Methods section. $\mathrm{n} / \mathrm{a}$ Confirmed

$\bigotimes$ The exact sample size $(n)$ for each experimental group/condition, given as a discrete number and unit of measurement

Х A statement on whether measurements were taken from distinct samples or whether the same sample was measured repeatedly

The statistical test(s) used AND whether they are one- or two-sided

Only common tests should be described solely by name; describe more complex techniques in the Methods section.

$\square$ A description of all covariates tested

$\square$ \ A description of any assumptions or corrections, such as tests of normality and adjustment for multiple comparisons

$\square$ A full description of the statistical parameters including central tendency (e.g. means) or other basic estimates (e.g. regression coefficient)

AND variation (e.g. standard deviation) or associated estimates of uncertainty (e.g. confidence intervals)

$\square$ For null hypothesis testing, the test statistic (e.g. $F, t, r$ ) with confidence intervals, effect sizes, degrees of freedom and $P$ value noted

Wive $P$ values as exact values whenever suitable.

Х $\square$ For Bayesian analysis, information on the choice of priors and Markov chain Monte Carlo settings

Х $\square$ For hierarchical and complex designs, identification of the appropriate level for tests and full reporting of outcomes

$\square$ Estimates of effect sizes (e.g. Cohen's d, Pearson's $r$ ), indicating how they were calculated

Our web collection on statistics for biologists contains articles on many of the points above.

\section{Software and code}

Policy information about availability of computer code

$\begin{array}{ll}\text { Data collection } & \text { Flow cytometry: FlowLogic V7 } \\ & \text { Metabolomics: Progenesis Ql software v2.3 }\end{array}$

Lipidomics: SCIEX Lipidomics Workflow Manager v1.0.5.0

Data analysis Data analysis was performed using R version 3.6.0-3.6.3. Key packages used were: 'sva' v3.34, 'limma' v3.42.2, DESeq2 1.26.0, phenotest v1.34.0, MetID v0.2.0, MetaboAnalystR 2.0.4, 'Diffbind' 2.14, caret 6.0-86,'nnet' 7.3-13, 'randomForest' 4.6-14, 'gbm' 2.1.5, kernlab 0.9-29,'sparseLDA' 0.1-9, 'party' 1.3-4, 'LiblineaR' 2.10-8, 'pROC' 1.16.2.

Standalone software used: Trimgalore 0.4.4, STAR 2.5.0a, subread 1.5.3, NetworkAnalyst 3.0, IMPaLA v12, LION (access on 03-24-2020), NGmerge0.2, bowtie2 2.2.6, samtools 1.5, HOMER 4.11, QIAGEN's Ingenuity Pathway analysis (IPA QIAGEN Redwood City), ImageJ 1.53. NucleoATAC 0.3.4, GREAT 4.0.4

Custom code developed for this study is available on Github at https://github.com/BenayounLaboratory/Neutrophil_Omics_2020 
Policy information about availability of data

All manuscripts must include a data availability statement. This statement should provide the following information, where applicable:

- Accession codes, unique identifiers, or web links for publicly available datasets

- A list of figures that have associated raw data

- A description of any restrictions on data availability

Sequencing data has been submitted to the Sequence Read Archive (SRA) accessible through BioProject PRJNA630663.

Untargeted metabolomics data was uploaded to metabolomics workbench DataTrack ID 2089.

The lipidomics data is available as Supplemental Table S7 and was deposited in Figshare (https://doi.org/10.6084/m9.figshare.14524278).

Raw flow cytometry data was deposited to Figshare (doi.org/10.6084/m9.figshare.14043938.v1, doi.org/10.6084/m9.figshare.14043932.v1, doi.org/10.6084/

m9.figshare.14043929.v1, doi.org/10.6084/m9.figshare.14043926.v1, and doi.org/10.6084/m9.figshare.14043923.v1).

Western Blot raw and cropped images have been deposited to Figshare (https://doi.org/10.6084/m9.figshare.14154665).

We reanalyzed publicly available neutrophil RNA-seq data from GEO Datasets GSE1248296 (6 weeks-old mouse spleen neutrophil samples), and GSE145231 (human blood neutrophil samples), and human neutrophil DIA proteomics data (https://www.ncbi.nlm.nih.gov/pmc/articles/PMC6442368/

bin/141289_1_supp_251888_pjgfps.xlsx). Functional gene annotations were obtained from ENSEMBL Biomart (https://www.ensembl.org/biomart/martview/), the Molecular Signature Database (http://www.gsea-msigdb.org/gsea/msigdb/collections.jsp) and the Harmonizome (https://maayanlab.cloud/Harmonizome/ download). Mass-spectrometry peaks were identified by matching experimental $\mathrm{m} / \mathrm{z}$ and fragmentation spectra to publicly available databases including $\mathrm{HMDB}$ (http://www.hmdb.ca/), MoNA (http://mona.fiehnlab.ucdavis.edu/) and MassBank (http://www.massbank.jp/).

\section{Field-specific reporting}

Please select the one below that is the best fit for your research. If you are not sure, read the appropriate sections before making your selection.

\Life sciences Behavioural \& social sciences Ecological, evolutionary \& environmental sciences

For a reference copy of the document with all sections, see nature.com/documents/nr-reporting-summary-flat.pdf

\section{Life sciences study design}

All studies must disclose on these points even when the disclosure is negative.

Sample size

No statistical analysis was performed to pre-determine sample size.

For genomics, we used 3-5 replicates per biological group. These numbers have previously been used to achieve statistical differences in aging 'omics' studies (PMID: 24792119; PMID: 23810552; PMID: 26321660; PMID: 27135165;PMID: 28351383).

For other experiments, since effect size and variability was unknown prior to experiments, we used samples from 2-4 independent cohorts of animals to exclude cohort-specific effects (known to occur in aging studies [PMID: 27923560]) and technical noise. For aging animal analyses, cohorts of 16-20 were obtained from the NIA colony, and only animals surviving to the experimental endpoint were used. These group sizes and cohort numbers are consistent with what has been used in aging research to achieve significance with noisy outcomes (PMID: 29590078;PMID: 31270459;PMID: 31645721;PMID: 24779584;PMID: 33796866).

The number of sample is explained in the text and by showing all experimental points on the relevant plots.

Data exclusions

No data passing quality control checks were excluded.

Replication

The "omics" datasets comprise independent biological samples. 'Omic' datasets from lab animals are not usually replicated in independent cohorts (see for instance PMID: 24792119; PMID: 23810552; PMID: 26321660; PMID: 27135165;PMID: 28351383). Thus, we did not replicate our metabolomics, lipidomics and ATAC-seq datasets on independent cohorts. RNA-seq was independently replicated on a smaller cohort, since neutrophils are known to be RNA-poor, which could add more than usual biological variability. The LPS/ELANE experiment was obtained from 3 independent cohorts. The Western blotting data was obtained from independent animals from 2 independent cohorts. All flow cytometry data was obtained from at least 2 independent cohorts (except for the whole bone marrow control for c-Kit staining, used to confirm antibody reactivity, from one single cohort). All replication attempts were successful.

Randomization For each experiment, animals were processed in an alternating/snaking order rather than in large homogeneous groups to minimize group, batch and circadian effects. For observational aging studies, no randomization based on group allocation is possible since groups are biologically determined. For the LPS inejction experiments, animals were randomly allocated to the PBS or LPS group for a euthanasia time point, on a cage-level basis (i.e. animals from the same cage randomly attributed to LPS vs. PBS injections; for each cohort, co-housed animals were used for the same time point; cages attributed to a specific euthanasia time point randomly), independently for each of the 3 cohorts. This process was performed independently for each of the 3 cohorts for LPS-induced endotoxemia.

Blinding

Blinding was not relevant to the studies conducted here, as the data was collected by automated systems (i.e. next-generation sequencing, mass-spectrometry, flow cytometry) or in numeric form (i.e. ImageJ quantification of Western Blot bands, ELISA absorbance values), which cannot be influenced by subjectivity from the experimenter. In addition, 'omic' analyses require knowledge of sample meta-data to be able to run analyses. 


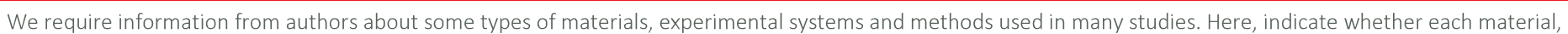

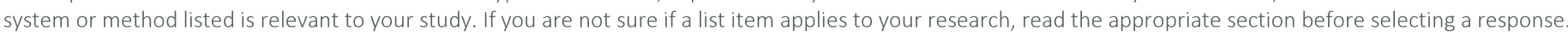

\begin{tabular}{|c|c|c|c|}
\hline \multicolumn{2}{|c|}{ Materials \& experimental systems } & \multicolumn{2}{|c|}{ Methods } \\
\hline $\mathrm{n} / \mathrm{a}$ & Involved in the study & $n / a$ & Involved in the study \\
\hline & Х Antibodies & Х & $\square$ ChIP-seq \\
\hline Х & Eukaryotic cell lines & & \ Flow cytometry \\
\hline Х & $\square$ Palaeontology and archaeology & Х & MRI-based neuroimaging \\
\hline & Х Animals and other organisms & & \\
\hline Х & Human research participants & & \\
\hline Х & Clinical data & & \\
\hline Х & Dual use research of concern & & \\
\hline
\end{tabular}

\section{Antibodies}

Antibodies used

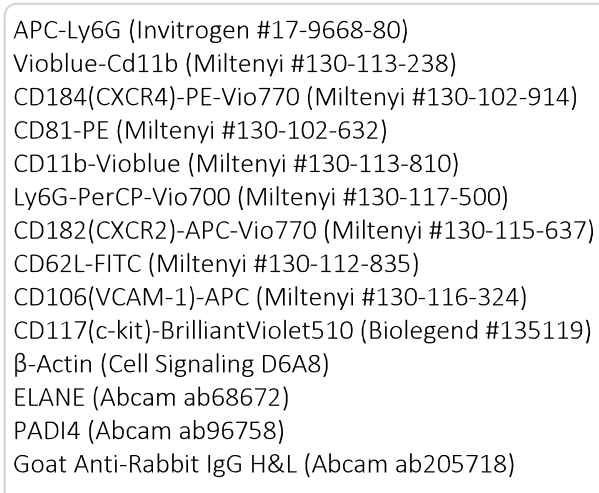

\section{- APC-Ly6G (Invitrogen \#17-9668-80)}

Validated for flow cytometry on mouse bone marrow cells based on the ThermoFisher website. The website also states "This 1A8Ly6g antibody has been tested by flow cytometric analysis of mouse bone marrow cells."

(https://www.thermofisher.com/antibody/product/Ly-6G-Antibody-clone-1A8-Ly6g-Monoclonal/17-9668-82)

- Vioblue-Cd11b (Miltenyi \#130-113-238)

Validated for flow cytometry on mouse splenocytes based on the Miltenyi website. Validated for specificity in comparison to known $\mathrm{M} 1 / 70$ clone.

(https://www.miltenyibiotec.com/US-en/products/cd11b-antibody-anti-human-mouse-m1-70-15-11-5.html)

- CD184(CXCR4)-PE-Vio770 (Miltenyi \#130-102-914)

Validated for flow cytometry on mouse thymocytes based on the Miltenyi website. Validated for specificity in comparison to known 2B11 clone.

(https://www.miltenyibiotec.com/US-en/products/cd184-cxcr4-antibody-anti-mouse-reafinity-rea107.html)

- CD81-PE (Miltenyi \#130-102-632)

Validated for flow cytometry on mouse postnatal brain cells based on the Miltenyi website.

(https://www.miltenyibiotec.com/US-en/products/cd81-antibody-anti-mouse-rat-eat2.html)

- CD11b-Vioblue (Miltenyi \#130-113-810)

Validated for flow cytometry on mouse splenocytes based on the Miltenyi website, in comparison to corresponding REA control antibodies.

(https://www.miltenyibiotec.com/US-en/products/cd11b-antibody-anti-mouse-reafinity-rea592.html)

- Ly6G-PerCP-Vio700 (Miltenyi \#130-117-500)

Validated for flow cytometry on mouse bone marrow cells based on the Miltenyi website, in comparison to corresponding REA control antibodies. Validated for specificity in comparison to known $1 \mathrm{~A} 8$ clone.

(https://www.miltenyibiotec.com/US-en/products/ly-6g-antibody-anti-mouse-reafinity-rea526.html)

- CD182(CXCR2)-APC-Vio770 (Miltenyi \#130-115-637)

Validated for flow cytometry on mouse bone marrow cells based on the Miltenyi website, in comparison to corresponding REA 
control antibodies and in combination with Anti-Gr1 antibodies.

(https://www.miltenyibiotec.com/US-en/products/cd182-cxcr2-antibody-anti-mouse-reafinity-rea942.html)

- CD62L-FITC (Miltenyi \#130-112-835)

Validated for flow cytometry on mouse splenocytes based on the Miltenyi website, in comparison to corresponding REA control antibodies and in combination with CD4 antibodies.

(https://www.miltenyibiotec.com/US-en/products/cd62l-antibody-anti-mouse-reafinity-rea828.html)

- CD106(VCAM-1)-APC (Miltenyi \#130-116-324)

Validated for flow cytometry on mouse bone marrow cells based on the Miltenyi website, in comparison to corresponding REA control antibodies and in combination with CD11b antibodies.

(https://www.miltenyibiotec.com/US-en/products/cd106-vcam-1-antibody-anti-mouse-reafinity-rea971.html)

- CD117(c-kit)-BrilliantViolet510 (Biolegend \#135119)

Validated for flow cytometry on mouse bone marrow cells based on the Biolegend website, compared to an isotype control. (https://www.biolegend.com/en-us/products/brilliant-violet-510-anti-mouse-cd117-c-kit-antibody-8482)

- $\beta$-Actin (Cell Signaling D6A8)

Validated for Western blot on mouse and human cell lines based on the Cell Signaling website, with the band migrating at the expected size by Western blot. The website also states " $\beta$-Actin (D6A8) Rabbit mAb recognizes endogenous levels of total $\beta$-actin protein. Due to the high sequence identity between the cytoplasmic actin isoforms, $\beta$-actin and cytoplasmic $\gamma$-actin, this antibody may cross-react with cytoplasmic $\gamma$-actin. It does not cross-react with $\alpha$-skeletal, $\alpha$-cardiac, $\alpha$-vascular smooth, or $\gamma$-enteric smooth muscle isoforms."

(https://www.cellsignal.com/products/primary-antibodies/b-actin-d6a8-rabbit-mab/8457)

- ELANE (Abcam ab68672)

Validated for Western blot, including on mouse bone marrow cells and the purified human protein, based on the Abcam website, with the band migrating at the expected size by Western blot. The website also states "Use a concentration of $1 \mu \mathrm{g} / \mathrm{ml}$. Detects a band of approximately $26 \mathrm{kDa}$ (predicted molecular weight: $29 \mathrm{kDa}$ ). In our hands, when tested in western blot, this product typically gives a weaker signal in human tissue lysates compared to human cell lines and mouse lysates."

(https://www.abcam.com/neutrophil-elastase-antibody-ab68672.html)

- PADI4 (Abcam ab96758)

Validated for Western blot, on human HEK293T-overexpressing cells (vs. untransfected HEK293T) as well as purified human protein, predicted to cross-react with mouse Padi4, based on the Abcam website, with the band migrating at the expected size by Western blot. (https://www.abcam.com/padi4--pad4-antibody-ab96758.html)

- Goat Anti-Rabbit IgG H\&L (Abcam ab205718)

Validated to work for Western blot as a secondary antibody for beta-actin. In addition, the specificity of the antibody to rabbit IgGs was confirmed using sandwich ELISA.

(https://www.abcam.com/goat-rabbit-igg-hl-hrp-ab205718.html)

\section{Animals and other organisms}

Policy information about studies involving animals; ARRIVE guidelines recommended for reporting animal research

Laboratory animals

For 'omics' analyses, male and female C57BL/6Nia mice (4-5 and 20-24 months old animals) were obtained from the National Institute on Aging (NIA) colony at Charles Rivers. For the sepsis assays in young animals, male and female C57BL/6J mice (3-4 months old animals) were obtained from Jackson Laboratories. Both Charles Rivers and Jackson Laboratories have specific-pathogen-free (SPF) facilities. Animals were acclimated at the SPF animal facility at USC for 2-4 weeks before any processing. The facility is on a $12-$ hour light/dark cycle, and animal housing rooms are maintained at $72 \mathrm{~F}$ and $30 \%$ humidity. All animals were euthanized between 8-11am for 'omics' experiments, flow cytometry and NETosis assays. For the sepsis model experiments, animals were injected in the morning, and euthanized between 4-6pm. In all cases, animals were euthanized using a "snaking order" across all groups to minimize batch-processing confounds due to circadian processes (including potential confounds due to neutrophil cellular "age"). All animals were euthanized by $\mathrm{CO} 2$ asphyxiation followed by cervical dislocation.

Wild animals

No wild animals were used in this study.

Field-collected samples

No field collection of samples was conducted in this study.

Ethics oversight

All animals were treated and housed in accordance with the Guide for Care and Use of Laboratory Animals. All experimental procedures were approved by the University of Southern California's Institutional Animal Care and Use Committee (IACUC) and are in accordance with institutional and national guidelines. Samples were derived from animals on approved IACUC protocols \#20770, \#20804 and \#21004.

Note that full information on the approval of the study protocol must also be provided in the manuscript. 
Plots

Confirm that:

\The axis labels state the marker and fluorochrome used (e.g. CD4-FITC).

\The axis scales are clearly visible. Include numbers along axes only for bottom left plot of group (a 'group' is an analysis of identical markers).

\All plots are contour plots with outliers or pseudocolor plots.

$\bigotimes$ A numerical value for number of cells or percentage (with statistics) is provided.

\section{Methodology}

Sample preparation

Instrument

\section{Software}

Cell population abundance

Gating strategy
The long bones of each mouse were harvested and kept on ice in D-PBS (Corning) supplemented with 1\% Penicillin/ Streptomycin (Corning) until further processing. Muscle tissue was removed from the bones, and the bone marrow from cleaned bones was collected into clean tubes. Red blood cells from the marrow were removed using Red Blood Cell Lysis buffer (Miltenyi Biotech \#130-094-183), according to the manufacturer's instructions, albeit with no vortexing step to avoid unscheduled neutrophil activation. Neutrophils were isolated from other bone marrow cells using magnetic-assisted cell sorting (Miltenyi Biotech kit \#130-097-658). Viability and yield were assessed using trypan blue exclusion and an automated COUNTESS cell counter (Thermo-Fisher Scientific).

**Neutrophils purity estimate by flow cytometry**

We used aging C57BL/6Nia mice from two independent cohorts to estimate potential differences in neutrophil purity with respect to sex and age. After an Fc-blocking step (Miltenyi Biotech \#130-092-575), MACS-purified neutrophils were then stained using APC-Ly6G (Invitrogen \#17-9668-80) and Vioblue-Cd11b (Miltenyi Biotech \#130-113-238) at a 1:50 dilution according to the manufacturer's instructions

**Purified neutrophil heterogeneity estimate by flow cytometry**

After isolation by MACS, $2.5 \times 105$ cells were washed once by adding $1 \mathrm{~mL}$ of PBS/EDTA $0.1 \%$ BSA (i.e. MACS resuspension buffer) in a $5 \mathrm{~mL}$ polystyrene round bottom tube (Falcon Cat \#352054), then centrifuged 300g for 10 minutes at 4C. After an Fc-blocking step (Miltenyi Biotech \#130-092-575), MACS-purified neutrophils were then stained with antibodies for 20 minutes at $4 \mathrm{C}$, and excess antibody was washed away using resuspension buffer.

Due to the low amount of c-kit+ events in MACS-purified neutrophils, we also ran whole bone marrow (pooled by group in one cohort) with the same scheme to confirm that positive labelling by this antibody occurs normally prior to purification.

MACS Quant Analyzer 10 (Miltenyi Biotech)

Flow cytometry results were processed using the FlowLogic V7 software.

Purity of neutrophils was determined as the fraction of $\mathrm{Cd} 11 \mathrm{~b}+/ \mathrm{Ly} 6 \mathrm{G}+$ cells.

Among live cell singlets, pro-neutrophils were defined as c-Kit+Ly6g-Cd81+, with subpopulation of pro-neutrophil 1 defined as c-Kit+Ly6G-Cd81+CD11b-CD106- and pro-neutrophils 2 as c-Kit+Ly6G-Cd81+CD11b+CD106+. Pre-neutrophils were defined as Cd11b+Ly6G+C-Kit+CxCr4+ cells. Immature neutrophils were defined as CD11b+Ly6G+c-Kit-Cxcr4-Cxcr2- cells, and mature neutrophils as $C D 11 b+L y 6 G+C-K i t-C x c r 4-C x c r 2+$. Fresh vs. "aged" subsets of mature neutrophils were defined respectively as CD11b+Ly6G+c-Kit-Cxcr4-Cxcr2+CD62L+vs. CD11b+Ly6G+c-Kit-Cxcr4-Cxcr2+CD62L-cells.

Gates were defined using a combined sample with equal amount of cells from all tested samples from each cohort, with (i) an unstained control and (ii) FMO controls to define gating thresholds.

$\bigotimes$ Tick this box to confirm that a figure exemplifying the gating strategy is provided in the Supplementary Information. 